\title{
A Model for the Development of Simple Cell Receptive Fields and the Ordered Arrangement of Orientation Columns through Activity- dependent Competition between ON- and OFF-Center Inputs
}

\author{
Kenneth D. Miller \\ Division of Biology, California Institute of Technology, Pasadena, California 91125
}

Neurons in the primary visual cortex of higher mammals respond selectively to light/dark borders of a particular orientation. The receptive fields of simple cells, a type of orientation-selective cell, consist of adjacent, oriented regions alternately receiving ON-center and OFF-center excitatory input. I show that this segregation of inputs within receptive fields can occur through an activity-dependent competition between ON-center and OFF-center inputs, just as segregation of inputs between different postsynaptic cells into ocular dominance columns appears to occur through activity-dependent competition between left-eye and right-eye inputs. These different outcomes are proposed to result, not from different mechanisms, but from different spatial structures of the correlations in neural activity among the competing inputs in each case. Simple cells result if $\mathrm{ON}$-center inputs are best correlated with other ON-center inputs, and OFF with OFF, at small retinotopic separations, but ON-center inputs are best correlated with OFF-center inputs at larger separations.

This hypothesis leads robustly to development of simple cell receptive fields selective for orientation and spatial frequency, and to the continuous and periodic arrangement of preferred orientation across the cortex. Input correlations determine the mean preferred spatial frequency and degree of orientation selectivity. Estimates of these correlations based on measurements in adult cat retina (Mastronarde, $1983 a, b)$ produce quantitative predictions for the mean preferred spatial frequencies of cat simple cells across eccentricities that agree with experiments (Movshon et al., 1978b). Intracortical interactions are the primary determinant of cortical organization. Simple cell spatial phases can play a key role in this organization, so arrangements of spatial phases

\footnotetext{
Received July 13, 1992; revised July 7, 1993; accepted July 13, 1993.

I thank M. P. Stryker for encouraging me to take these ideas seriously at an early stage, and him and C. Koch for providing facilities and support. D. J. C. MacKay, B. Mel, E. Niebur, R. Soodak, and M. P. Stryker provided useful discussions and, along with the reviewers, helpful comments on the manuscript. $B$ Chapman, U. Kaplan, D. Mastronarde, M. Meister, L. Palmer, P. Schiller, and $R$. Wong patiently answered many questions about their experimental results. $D$. Mastronarde also provided a computer program needed to interpret his results. I was supported at Caltech by a Del Webb Division of Biology Fellowship, and prior to that at UCSF by an N.E.I. Fellowship. Preliminary simulations were done at the San Diego Supercomputer Center, supported by the NSF.

Present address: Departments of Physiology and Otolaryngology, W. M. Keck Foundation Center for Integrative Neuroscience, and Neuroscience Graduate Program, University of California, San Francisco, CA 94143-0444. Correspondence should be addressed to Kenneth D. Miller, Department of Physiology, University of California, San Francisco, CA 94143-0444.

Copyright (C) 1994 Society for Neuroscience $0270-6474 / 94 / 140409-33 \$ 05.00 / 0$
}

and preferred orientations may need to be studied together to understand either alone. Possible origins for other cortical features including spatial frequency clusters, afferent ON/ OFF segregation, blobs, pinwheels, and opponent inhibition within simple cell receptive fields are suggested. A number of strong experimental tests of the hypothesis are proposed.

[Key words: Hebb synapse, simple cell, ON-center cell, OFF-center cell, orientation column, orientation tuning, spatial phase, spatial frequency, visual cortex, developmental model, synaptic competition, correlations]

Most neurons in the primary visual cortex of many mammals are tuned to respond to light/dark borders of a particular orientation (Hubel and Wiesel, 1959, 1962). The inputs that cortical neurons receive from the lateral geniculate nucleus (LGN), in contrast, respond well to bordcrs of all orientations and to nonoriented stimuli (Hubel and Wiesel, 1961).

The physiological origin of orientation selectivity in visual cortical responses remains uncertain. Hubel and Wiesel (1962) proposed that orientation selectivity may arise in cortical "simple cells." Simple cell receptive fields are composed of adjacent, oriented ON- and OFF-subregions. Each ON-subregion receives excitatory drive largely or exclusively from the ON-center input stream, and similarly for OFF-subregions (Schiller, 1982; Sherk and Horton, 1984; Ferster, 1988). The "Hubel-Wiesel model" proposes that these oriented subregions arise through the ordered arrangement of LGN inputs: "upon each simple-type cell there converge (LGN) fibres. . having 'on' or 'off' centres situated in the appropriate retinal regions" (Hubel and Wiesel, 1962). Much current evidence seems consistent with this model (e.g., Tanaka, 1983; Sherk and Horton, 1984; Ferster, 1987, 1988; Chapman et al., 1991).

The developmental origin of orientation selectivity is also uncertain. It has often been proposed that orientation selectivity develops through a process of activity-dependent synaptic modification. However, because orientation selectivity arises early in development, before birth in monkeys (Wiesel and Hubel, 1974) and before eye opening in kittens (e.g., Hubel and Wiesel, 1963; Albus and Wolf, 1984; Braastad and Heggelund, 1985) and ferrets (Chapman, 1991), it has not yet been possible to test this hypothesis directly. Many studies have shown that orientation selectivity, once established, is sharpened by neural activity, and have addressed whether preferred orientation can be altered by visual experience (reviewed in Movshon and Van Sluyters, 1981; Fregnac and Imbert, 1984; see also Braastad and Heggelund, 1985; Buisseret et al., 1988; Isley et al., 1990; Chapman, 1991). 
In this article I propose a developmental origin for a HubelWiesel model of simple cell receptive fields. I show that such receptive fields, and their organization into continuous and periodic arrangements of preferred orientation across the cortex, arise naturally through an activity-dependent, correlation-based synaptic competition between ON-center and OFF-center inputs to the visual cortex.

This result, in which two competing input types (ON- and OFF-center cells) converge onto the same postsynaptic cells, differs from the outcome often reported for correlation-based competition. Instead, such competition often results in the segregation of two input types onto different postsynaptic cells (reviewed in Constantine-Paton et al., 1990; Miller, 1990a). Wellknown examples include the segregation of inputs serving left and right eyes into eye-specific layers in the LGN and into ocular dominance patches in the geniculate-recipient layers of visual cortex (reviewed in Miller and Stryker, 1990; Shatz, 1990). Similarly, ON- and OFF-center retinal inputs segregate onto different postsynaptic cells in the LGN: ON-center LGN cells receive exclusively $\mathrm{ON}$-center input from the retina, and similarly for OFF-center LGN cells (Horton and Sherk, 1984; Schiller, 1984; Dubin et al., 1986; Mastronarde, 1987). In many species, ONand OFF-center inputs to LGN are further segregated into separate layers (Schiller and Malpeli, 1978; LeVay and McConnell, 1982; Conway and Schiller, 1983; Stryker and Zahs, 1983). This segregation of $\mathrm{ON}$ - and OFF-center inputs, like that of left-and right-eye inputs, appears to be activity dependent (Dubin et al., 1986; Hahm et al., 1991).

In contrast to the above cases, ON- and OFF-center inputs converge in the orientation-selective layers of the visual cortex. Convergence has been shown both physiologically, in that excitatory input from both ON-center and OFF-center streams drives responses in the great majority of oriented cortical cells (Schiller, 1982; Sherk and Horton, 1984), and anatomically, in that single oriented cortical cells in the cat have been shown to receive both types of monosynaptic geniculate input (Tanaka, 1983). In monkeys, parvocellular geniculate inputs innervate cortical layer $4 C \beta$, which is itself composed of center-surround, ON-center or OFF-center cells (Hubel and Wiesel, 1968; Blasdel and Fitzpatrick, 1984); it is not known whether ON- and OFFcenter streams converge in these cortical cells. If they do not, then in both cats and monkeys, the transition from center-surround to oriented organization is coincident with the transition from segregation to convergence of $\mathrm{ON}$ - and OFF-center streams.

Despite this convergence, ON- and OFF-center inputs segregate retinotopically within simple cell receptive fields. I will show that this can robustly result from a correlation-based synaptic competition between ON-center and OFF-center inputs. This competition is hypothesized to occur by precisely the same mechanism as that which has been shown in previous models (von der Malsburg and Willshaw, 1976; Miller et al., 1989) to lead to segregation between postsynaptic cells, as in ocular dominance segregation. The two types of outcome-(1) segregation between postsynaptic cells or (2) convergence onto the same postsynaptic cells combined with segregation within receptive fields-result from development under this mechanism given two different types of correlations among the input activities. I will also show that retinotopic segregation within receptive fields, when combined with lateral interactions between nearby cortical cells, is sufficient to explain the development and basic horizontal organization of cortical orientation selectivity.

This hypothesis explains why the transition from center-sur- round to oriented receptive fields should be coincident with the transition from segregation to convergence of ON-center and OFF-center input streams, and why the oriented cells at this transition point should largely be simple cells. It also simplifies our picture of development, by showing that synapses in cortex may be assumed to respond to the same developmental rules whether they are viewed as coming from ON-and OFF-center inputs or from left- and right-eye inputs, and by explaining why $\mathrm{ON}$ - and OFF-center inputs obeying these rules can segregate in LGN yet converge in cortex. The hypothesis also accounts for other aspects of receptive field structure, such as the preferred spatial frequencies of simple cells.

There have been many previous models for the activity-dependent development of orientation selectivity, beginning with von der Malsburg (1973). Most assumed a single type of input, and showed that oriented synaptic patterns could result from correlation-based development under exposure to oriented patterns of input activity, as in vision. Such models do not account for the development of segregated receptive field subregions, and do not address the development of orientation selectivity before visual experience. Linsker $(1986 \mathrm{a}-\mathrm{c})$ demonstrated that an activity-dependent process could yield oriented receptive fields with segregated subregions of excitatory and inhibitory inputs, without exposure to oriented input patterns. However, his results relied upon a model in which feedforward synapses could have either positive or negative strength, and upon careful tuning of certain nonbiological parameters that arise in such a model. Tanaka (1992) independently proposes that an ON/OFF competition may underlie the development of orientation selectivity. In his model, a cortical cell comes to receive only a single geniculate input, and receptive field structure derives from intracortical interactions, whereas in the present model, a simple cell's receptive field structure derives from the pattern of geniculate inputs received by the cell. These differences are further examined in the Discussion.

Before presenting the methods and results of the present study, it is necessary to review briefly the theoretical framework to be used.

\section{Theoretical framework: basic assumptions}

The simplest proposed mechanism for activity-dependent synaptic modification is a "Hebb synapse" (Hebb, 1949), that is, a synapse that is strengthened when there is correlation between presynaptic and postsynaptic activation or depolarization, and unchanged or weakened otherwise (Constantine-Paton et al. 1990; Bliss and Collingridge, 1993). Hebb synapses and related mechanisms, such as activity-dependent release and action of a diffusible modification factor (Bliss and Collingridge, 1993), can in many cases be described within a single mathematical framework (Miller et al., 1989). These mechanisms have in common the dependence of development on the correlations among the activities of the inputs.

I have studied these correlation-based mechanisms within the context of a simple class of models first used by von der Malsburg (1973), and subsequently used by many other theorists (e.g., Bienenstock et al., 1982; Linsker, 1986a-c; Miller et al., 1989; Tanaka, 1990, 1992; Miller, 1992a). These models share the following assumptions:

- The cortex, and each set of input neurons (e.g., ON, OFF) are modeled as 2-D layers of cells ( $1-D$ layers or a single postsyriaplic cell may also be used).

- The cortical wiring ensures that there is either (1) a "Mexican 
hat" interaction in the cortex, in which excitation of a given cortical location excites nearby locations and inhibits more distant locations, or (2) exclusively excitatory interactions between nearby cortical locations. This intracortical interaction via synaptic connections may be augmented or replaced by an interaction via diffusion of modification factors.

- The connections from each input cell to the cortex may be localized, as described by an "arbor" or "synaptic density" function.

- Input ccll activities $x_{i}$ determine cortical cell activities $y_{\text {, }}$ according to a linear or semilinear rule $y_{j}=f\left(\Sigma_{i} w_{j r} x_{i}+\Sigma_{i} b_{j i} y_{i}\right)$, where $w_{j i}$ is the synaptic weight from input cell $i$ to cortical cell $j, b_{i i}$ is the synaptic weight from cortical cell $i$ to cortical cell $j$, and $f$ is a linear or sigmoidal function. This activation rule may also be time dependent, but in this case an input pattern is considered to be sustained until an equilibrium is reached, and the equilibrium activity is governed by the given instantaneous equation.

- Modification of synapses from input cells occurs according to some Hebb-like or correlation-based rule, for example, $\frac{d}{d t} w_{j i}$ $\propto h_{r}\left(y_{j}\right) h_{i}\left(x_{i}\right)$, where $h_{i}$ and $h_{i}$ are functions, the symbol $\propto$ means "proportional to," and the $w_{j i}$ are subject to upper and lower bounds. A specific example is a covariance rule (Sejnowski, 1977) with hard limits on synaptic weights: $\frac{d}{d t} w_{j} \propto\left(y_{j}-\left\langle y_{j}\right\rangle\right)$ $\left(x_{i}-\left\langle x_{i}\right\rangle\right), 0<w_{j}<w_{\max } ; \frac{d}{d t} w_{j i}=0$, otherwise. Here, $\left\langle y_{j}\right\rangle$ and $\left\langle x_{i}\right\rangle$ are the time averages of $y_{j}$ and $x_{i}$, respectively, and $w_{\max }$ is a maximum value for synaptic weights. The postsynaptic function $h$ could also take into account the concentration of a diffusible modification factor that is released by cortical activity.

- Intracortical synapses are generally not treated as modifiable.

- A constraint or dynamical rule ensures that the synaptic changes are competitive: some synapses onto a cortical cell can become stronger only at the expense of other synapses ultimately becoming weaker, and vice versa.

Models of this type are reviewed in Miller (1992c). The validity of these assumptions is discussed there and in Miller (1990a) and Miller and Stryker (1990).

\section{Theoretical framework: elements of the model}

We have developed a mathematical framework that describes development in models of this type when two initially equivalent input projections compete to innervate a single output layer (Miller et al., 1989; Miller, 1990c). The initial development of a difference between the two input projections may be described by linear equations, even though the rules outlined above may include a number of nonlincarities. Thus, to study early pattern formation-for example, the initial pattern of segregation of ON- and OFF-center inputs within simple cell receptive fields - we may focus upon relatively simple, linear equations.

This early development of the input projections can be mathcmatically described as follows (Fig. 1). Hereafter I will take the input layer to be the LGN. The position of the presynaptic cell in the LGN is denoted by Greek letters $\alpha, \beta, \ldots$; the position of the postsynaptic cell in the cortex is denoted by letters $x, y$, $z, \ldots$ Note that these are each $2-\mathrm{D}$ vector positions. A direct conversion between positions in LGN and in cortex will always be assumed, for example, by describing both coordinate systems in common, retinotopic units. This allows definition of mixed quantities such as $z-\alpha$.

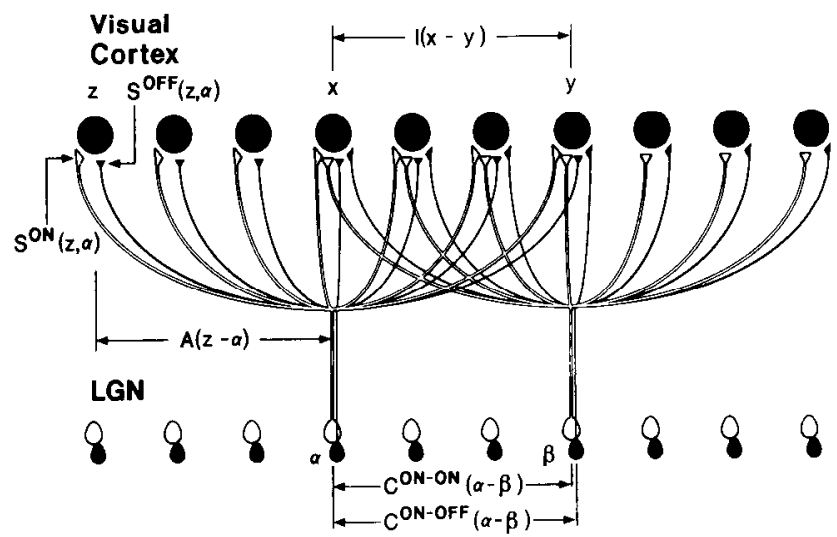

Figure 1. Elements of the model: ON (white) and OFF (black) cells in the LGN innervate cells in layer 4 of the visual cortex. $\alpha$ and $\beta$ label positions in the LGN and $x$ and $y$ label the retinotopically corresponding positions in the cortical layer; $z$ labels an additional cortical position. The figure illustrates the ON-center and OFF-center synaptic strengths, $S^{O N}$ and $S^{\text {OFF; }}$ the afferent correlation functions, $C^{\text {ONON }}$ and $C^{\text {ON.OFF }}$; the arbor function, $A$; and the intracortical interaction function, $I$. See introductory remarks for details

The ON-center synaptic strength from position $\alpha$ to position $z$ is $S^{\mathrm{ON}}(z, \alpha)$; similarly, $S^{\mathrm{OFF}}(z, \alpha)$ denotes OFF-center strength. These synaptic strengths are the dynamical elements of the mod$\mathrm{el}$; their time evolution represents cortical development. Intracortical connectivity is taken for simplicity to be fixed.

The development of $S^{O N}$ and $S^{O F}$ is expressed in terms of three measurable functions or sets of functions. The first is a set of correlation functions, here $C^{\text {NNON }}$, CON.OFF, and COFFOFF, describing the correlation between the activities of LGN cell pairs as a function of their center-type and separation. $C^{\mathrm{ON} O N}(\alpha$ $-\beta$ ) describes the correlation in activity of two ON-center cells from positions $\alpha$ and $\beta$, and similarly for the other functions. Orientation selectivity develops in many species before the onset of vision, as described above, so here the correlation functions describe the spontaneous neural activity in the absence of vision (dark activity). The second function is an arbor function that describes the distance over which a single geniculate input can arborize and make synapses in cortex. The arbor function $A(z-\alpha)$ is proportional to the number of synapses connecting the ON- or OFF-input from $\alpha$ to cortical position $z$. [The number of ON or OFF synapses, $A(z-\alpha)$, is regarded as fixed, while their summed strength $S^{\mathrm{ON}}(z, \alpha)$ or $S^{\mathrm{OF}}(z, \alpha)$ changes in time; anatomical plasticity can be modeled by assuming that synapses are removed when their strength becomes zero.]

The third function is an intracortical interaction function that describes how activity at one cortical location influences the development of correlated synapses on cells at nearby locations. This function, $I(x-y)$, describes the sign and magnitude of the interaction between points $x$ and $y$ in the cortex. The nature of this function depends on the biological mechanism proposed to underlie geniculocortical plasticity. In a Hcbbian mechanism, the intracortical interaction function is determined by intracortical synaptic connections: it is positive between two cortical locations that tend to excite one another, and negative between locations that tend to inhibit one another. For mechanisms that involve activity-dependent release and action of a modification factor, this function incorporates the spread of influence across 


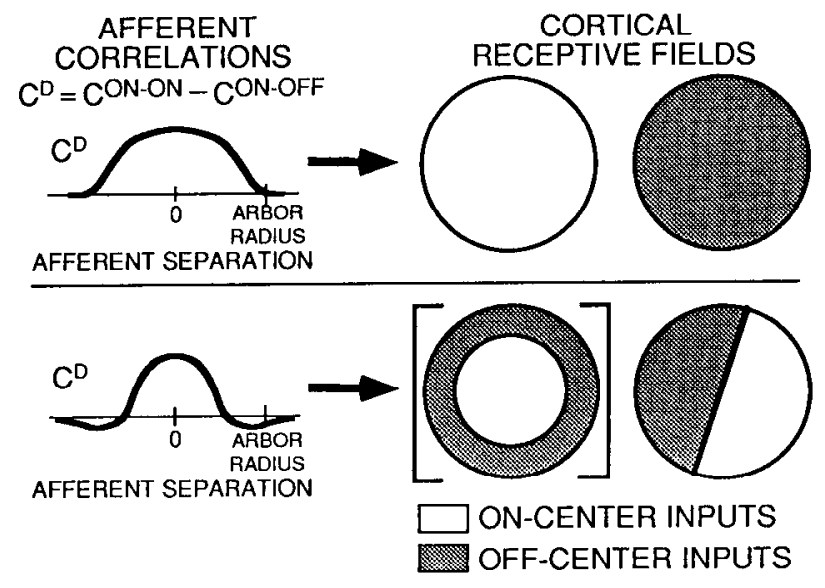

Figure 2. How correlations among afferent activities determine receptive field structure in the model. Left, Correlation function $C^{D}$ (vertical axis) between afferents with a given separation (horizontal axis). Right, Mature cortical receptive fields that result under the given $C^{D}$. Large circles indicate the set of afferent inputs received by a cortical cell after development is complete. White indicates $\mathrm{ON}$-center inputs, and shading indicates OFF-center inputs. Top, If $C^{\mathrm{D}}$ does not change sign within an arbor radius, segregation between postsynaptic cells occurs: a cortical cell comes to receive only ON-center (left) or only OFF-center (right) input. Bottom. If $C^{D}$ changes sign, so that at small separations sametype inputs are best correlated, but at larger separations within the arbor radius opposite-type inputs are best correlated, then receptive fields develop segregated ON-center and OFF-center subregions. Two (among many) alternative possible arrangements of such subregions are indicated. The correlation structure alone does not strongly distinguish between these. However, as will be seen, oriented arrangements (right) typically result; the brackets (lefi) indicate an alternative structure that rarely if ever occurs.

the cortex due to diffusion. In general this function depends on the detailed connectivity between specific cells, but for simplicity it is assumed to depend only on the separation between two cortical locations. In the Discussion, conclusions that apply to more general connectivity patterns are discussed.

\section{Theoretical framework: determinants of development}

The outcome of correlation-based development under the above framework has been qualitatively characterized (Miller et al., 1989; Miller, 1990a; Miller and Stryker, 1990; Miller and MacKay, 1994). This characterization assumes that the two input projections are equivalent, such that $C^{\mathrm{ON} . \mathrm{ON}}=\mathrm{C}^{\mathrm{OFF} . \mathrm{OFF}}$. This is a simplification, ignoring the greater dark activity of OFF-center inputs (Mastronarde, 1983b) and the apparently faster rate of development of the more active OFF population (Albus and Wolf, 1984; Braastad and Heggelund, 1985).

To describe the outcome of development, three additional quantities must be defined. First, the arbor radius is the radius in the cortex of the set of cortical cells that may receive input from a single LGN cell, that is, the radius over which $A(x)$ is nonzero. It is equivalently defined in the LGN as the radius of the set of LGN cells that connect to a single cortical cell. Second, the difference correlation function $C^{D}$ is defined by $C^{\triangleright}(\Delta) \equiv$ $C^{\text {ON.ON }}(\Delta)-C^{\text {ON.OFF }}(\Delta)$. $C^{D}(\Delta)$ tells the degree to which two inputs of the same center-type, with retinotopic separation $\Delta$, are better correlated with one another than with an input of the opposite center-type at the same separation $\Delta$. Third, because the model studies only the development of the afferent inputs, and does not consider mechanisms of cortical integration, I will use "receptive field," in describing model predictions and re-
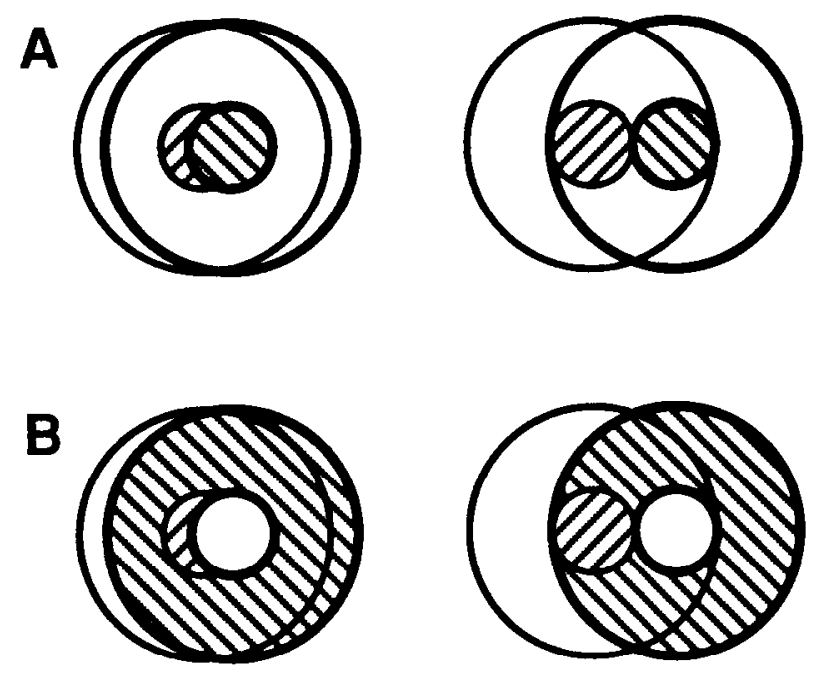

Figure 3. Correlations between ON- and OFF-center receptive fields at varying retinotopic separations. Stripes signify ON-regions; white. OFF-regions. Consider correlations in dark activity, due to shared photoreceptor input (Mastronarde, 1983b) as well as to intrinsic LGN connectivity. $A$, Two $O N$-center receptive fields. Left, At small retinotopic separations, ON-centers overlap and OFF-surrounds overlap. Hence, the cells would be likely to receive common input frequently and to be well correlated. Right, At larger retinotopic separations, the ON-center of each receptive field overlaps the OFF-surround of the other, so the two cells would be expected to receive common input rarely and thus to be poorly correlated or anticorrelated. $B, A n O N$-center and an OFFcenter cell at the same two retinotopic separations. The situation is reversed from $A$. Left, Anticorrelation is expected. Right, Better correlation is expected than at similar separations in $A$. This suggests the plausibility of a Mexican hat correlation structure, as in Figure 2, bottom.

sults, to mean the set of afferent inputs to a cortical cell. The basis for comparing model receptive fields, so defined, to experimental receptive fields, defined in terms of the response to visual stimulation, is considered in the Methods.

Two rules determine the outcome of development within the model framework. The first rule accounts for receptive field structure: cortical cells tend to develop receptive fields consisting of a subset of inputs that are as mutually correlated as possible. The difference correlation function, $C^{\circ}$, is the key factor determining which subset is most mutually correlated (Fig. 2) (other factors can also play a role; see Miller and MacKay, 1994). Two forms of $C^{2}$ that lead to qualitatively different receptive field structures can be distinguished. First, suppose $C^{\prime}$ is non-negative, so that two same-type inputs are better correlated than two opposite-type inputs at all separations (Fig. 2, top). Then the most correlated subset of inputs generally consists of inputs of a single type, so all-ON or all-OFF receptive fields develop. This represents segregation of two competing input populations onto different postsynaptic cells, as in the LGN or in ocular dominance segregation. Second, suppose (1) $C$ changes sign with distance, so that at small distances same-type inputs are best correlated but at larger distances opposite-type inputs are best correlated; and (2) this sign change occurs within the arbor radius (Fig. 2, bottom). I will refer to this as a "Mexican hat" correlation structure between $\mathrm{ON}$ - and OFF-center inputs. Then the receptive field of a cortical cell will develop segregated ONcenter and OFF-center subfields with widths that best match the sign changes in $\mathrm{C}^{\mathrm{D}}$. This represents convergence of two competing populations onto the same postsynaptic cells, but segregation within receptive fields, as in cortical simple cells. 
Development under the Mexican hat form of $C^{D}$ will be the focus of the present study.

Figure 3 demonstrates why a Mexican hat structure between $\mathrm{ON}$ - and OFF-center inputs is plausible. Suppose that correlations in dark activity are due to common photoreceptor input that excites "overlying" ON-subregions and inhibits overlying OFF-subregions (Mastronarde, 1983a,b). At small retinotopic separations (Fig. 3, left), like-center inputs have overlapping receptive fields, receive common photoreceptor input, and are therefore correlated in their activities, whereas opposite-center types have overlapping $\mathrm{ON}$ - and OFF-subregions and hence are anticorrelated. At larger separations (Fig. 3, right), two cells of opposite center types have greater overlap of ON-subregions and of OFF-subregions than two cells of the same center type, so the two cells of opposite type appear likely to be better correlated. The argument at larger separations must be taken with caution, because in adults dark-adapted cells have larger centers and weaker surrounds than light-adapted cells, at least in the retina (Enroth-Cugell and Lennie, 1975; Barlow and Levick, 1976); there are conflicting reports as to whether this is also true in the LGN (Virsu et al., 1977; Kaplan et al., 1979). Thus, the argument only suggests plausibility; measurement of LGN dark correlations is needed. The existing biological evidence on dark correlations is addressed in the Discussion.

The second developmental rule accounts for the formation of cortical maps: cortical cells tend to develop receptive fields that are as correlated as possible with other cortical receptive fields at mutually excitatory distances across the cortex, but as anticorrelated as possible with other receptive fields at mutually inhibitory distances. For the case of ocular dominance segregation (as in Fig. 2, top), if interactions are excitatory at small distances, this rule leads receptive fields to develop in ocular dominance patches, where the width of a left-eye patch plus a right-eye patch best matches the sign changes of the intracortical interaction function (Miller et al., 1989). For the present case (Fig. 2, bottom), the implications of this rule are more complicated, and will be discussed in greater detail below. However, it is this rule that leads oriented cells, if they develop, to have an ordered spatial arrangement across the cortex.

In summary, the hypothesis of this article is that the development of simple cell receptive fields and cortical maps may result from the combination of (1) an activity-dependent competition between $\mathrm{ON}$ - and OFF-center inputs to simple cells, via a Hebbian or other correlation-based mechanism of synaptic modification; (2) a Mexican hat correlation structure in the dark activity of these ON- and OFF-center inputs; and (3) intracortical interactions that couple the competitions occurring on different cortical cells.

Short accounts of this work have appeared previously (Miller, 1989b, 1990b, 1992a-c; Stryker et al., 1990).

\section{Methods}

The methods of simulation used here are similar to those of previous work (Miller, 1989a, 1992a; Miller et al., 1989). Previously published accounts were very brief, so a complete account is given here.

\section{The equation studied}

I will use the notation defined in Figure 1 and associated text. The equation studied is derived in Miller (1990c) and Miller et al. (1989). It consists of three pieces. First, the unconstrained ("U") equation that describes correlation-based development under Hebbian or similar rules is

$$
\begin{aligned}
\left.\frac{d}{d t}\right|_{\mathrm{U}} S^{\mathrm{ON}}(x, \alpha)= & \lambda A(x-\alpha) \sum_{y, \beta} I(x-y) \\
& \cdot\left[C^{\mathrm{UN}, \mathrm{UN}}(\alpha-\beta) S^{\mathrm{ON}}(y, \beta)+C^{\mathrm{ON}, \mathrm{OFF}}(\alpha-\beta) S^{\mathrm{OFF}}(y, \beta)\right] .
\end{aligned}
$$

$\frac{d}{d t} l_{U} S^{\mathrm{OFF}}$ is defined similarly, with $\mathrm{ON}$ and OFF exchanged everywhere. In words, this equation says the following: modification of synaptic strength is determined by the linear sum of the effects of all other synapses; the effect exerted by the synapses from $\beta$ to $y$ on those from $\alpha$ to $x$ is the product of their strength $S(y, \beta)$, their correlation $C(\alpha-$ $\beta$ ) with the synapses being modified, and their influence $I(x-y)$ on those synapses via intracortical connections and/or diffusion of modulatory factors. The arbor function, $A(x-\alpha)$, tells the number of synapses being modified.

Second, this equation is constrained to conserve the total synaptic weight over each postsynaptic cell (von der Malsburg, 1973; Miller and MacKay, 1994). This constraint models the fact that biological development under a correlation-based rule is competitive; that is, the growth of one input is detcrmined not simply by its own activity but by its activity relative to that of competing inputs (Wiesel and Hubel, 1965; Guillery, 1972; Stryker and Strickland, 1984). A conservation rule ensures that, if one set of cooperating inputs gains in synaptic strength, competitors must lose synaptic strength. The rule is not intended to model the biological mechanism by which competition is achieved, about which little is known. The resulting constrained equation is

$$
\frac{d}{d t} S^{O N}(x, \alpha)=\left.\frac{d}{d t}\right|_{\mathrm{U}} S^{\mathrm{ON}}(x, \alpha)-\epsilon(x) A(x-\alpha),
$$

where

$$
\epsilon(x)=\frac{1}{2 \sum_{\beta} A(x-\beta)} \cdot \sum_{\beta}\left[\left.\frac{d}{d t}\right|_{U} S^{\mathrm{ON}}(x, \beta)+\left.\frac{d}{d t}\right|_{U} S^{\mathrm{OFF}}(x, \beta)\right] .
$$

The equation for $\frac{d}{d t} S^{\text {(oF }}$ is Equation 2 with "ON" changed to "OFF." The second term in Equation 2 subtracts an equal amount from each synapse on a given cortical cell, and thus an amount proportional to the number of synapses $A(x-\alpha)$ from each total synaptic strength $S^{\mathrm{ON}}(x, \alpha)$ or $S^{\mathrm{OFF}}(x, \alpha)$, so as to set $\Sigma_{\text {ct }}\left[\frac{d}{d i} S^{\mathrm{ON}}(x, \alpha)+\frac{d}{d t} S^{\mathrm{OFF}}(x, \alpha)\right]=0$ for each cortical cell $x$.

Third, synapses cannot have less than 0 strength or more than some maximal strength, so weight limits are imposed: $0 \leq S^{\mathrm{ON}}(x, \alpha) \leq$ $s_{\max } A(x-\alpha)$, where $s_{\max }$ is an upper bound on synaptic weights. The equation studied in this article is Equation 2, subject to these weight limits.

The conservation rule (Eq. 2) is enforced subtractively, that is, by subtracting an equal amount from each synapse. Alternatively, such a constraint could be enforced by a multiplicative renormalization. The differences between these two methods are discussed in Miller and MacKay (1994). For the present model, there is little difference in outcome between the two. The major difference is that a subtractively enforced constraint leads all synaptic strengths eventually to reach either 0 or the maximum allowed strength, whereas a multiplicatively enforced constraint results in graded synaptic strengths. Thus, in the present simulations, all synaptic strengths approach the upper or lower limiting value, but this is an artifact of the constraint method and does not constitute a prediction or essential feature of the model.

\section{The architecture}

For purposes of simulation, the cortex, the ON-center inputs, and the OFF-center inputs are each modeled as 32 by 32 layers of neurons (Fig. 4). Each ON or OFF cell projects to a circular arbor of cortical cclls, centered about the retinotopically corresponding cell in the cortical grid. The arbor diameter is $\mathbf{1 3}$ grid intervals unless otherwise stated, yielding a total of 280,576 geniculocortical synapses (137 ON and 137 OFF synapses per cortical cell, 1024 cortical cells). To avoid boundary effects, periodic boundary conditions are used, so that the leftmost and rightmost columns of each grid are adjacent, as are the bottom and top rows. One cortical grid interval can be taken to represent roughly $100 \mu \mathrm{m}$ (see Fig. 15), in which case the simulation is of a roughly $3 \mathrm{~mm}$ by $3 \mathrm{~mm}$ patch of cortex with arbors around $1 \mathrm{~mm}$ in diameter.

The grid and arbor sizes are selected as follows. The grid size must 


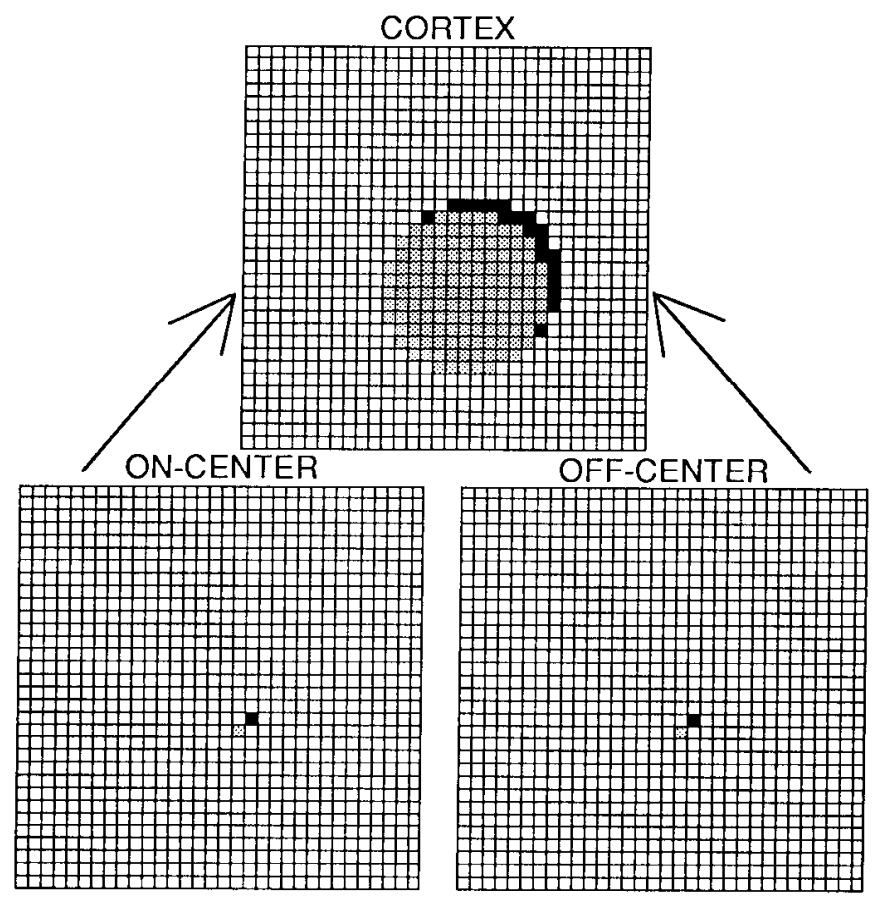

Figure 4. Grids used for simulations. A layer of ON-center and of OFF-center LGN inputs and a cortical recipient layer are each represented by 32 by 32 grids of cells. Each geniculate cell connects to a circle of diameter 13 grid intervals of cortical cells, centered about the retinotopically corresponding cortical position. Retinotopically corresponding cells in the two input layers (dark cells) connect to the same set of cortical cells, and adjacent input cells (gray) connect to adjacent and partially overlapping sets of cortical cells.

be a power of 2 because calculations use the fast Fourier transform, as described below. The largest grid size that can be accommodated is 32 , due to memory limitations. To study development of orientation selectivity, the arbor must be large enough that individual receptive fields can reliably develop significant internal structure; a diameter of 9 is about the minimum acceptable, but larger arbors yield more regular receptive field structure. At the same time, the arbor diameter should be significantly smaller than the grid width, so that the effects of restricting connectivity to a finite arbor can be seen. Parameters should also be chosen so that the grid is significantly larger than the width that emerges for an orientation hypercolumn (i.e., for a complete cycle of orientations; Hubel and Wiesel, 1974), so that periodic boundary conditions have minimal impact on hypercolumn formation.

\section{The functions used}

In choosing the functions $A, C_{,}$, and $I$ that define the model, the following considerations apply. Both theoretical and computational studies consistently find that, for the simple functional forms used here (i.e., falling smoothly to zero with distance, either monotonically or with a small number of oscillations), only very general features are important to the results: whether the function oscillates or falls monotonically to zero, the width if it falls monotonically, or the peak of its Fourier transform if it oscillates. Thus, certain fixed, simple forms of the functions were chosen, limiting the parameter space exploration to these relevant features. Most parameters are assigned "default" values; these were used in all cases where not specifically stated otherwisc. Typical cxamples of the functions used are shown in Figure 5.

The arbor function $A(x)$ is proportional to the overlap in area of a circle of radius $r$, and a circle of radius $c_{1} r$, with centers separated by $|x|$ (intended as a crude model of the overlap of geniculocortical terminal arbors with cortical dendritic arbors). It is set to zero for $|x|>$ $D_{1} / 2$, where $D_{1}$ is the "arbor diameter." Default parameters are $D_{4}=$ $13, r_{1}=(D-1) / 2, c_{1}=0.5$. The fact that the arbor function tapers, rather than being constant within the circle of radius $D_{t} / 2$, has little effect in most parameter regimes (see Fig. 10). Both analytic and computational results suggest that the role of the arbor function is primarily
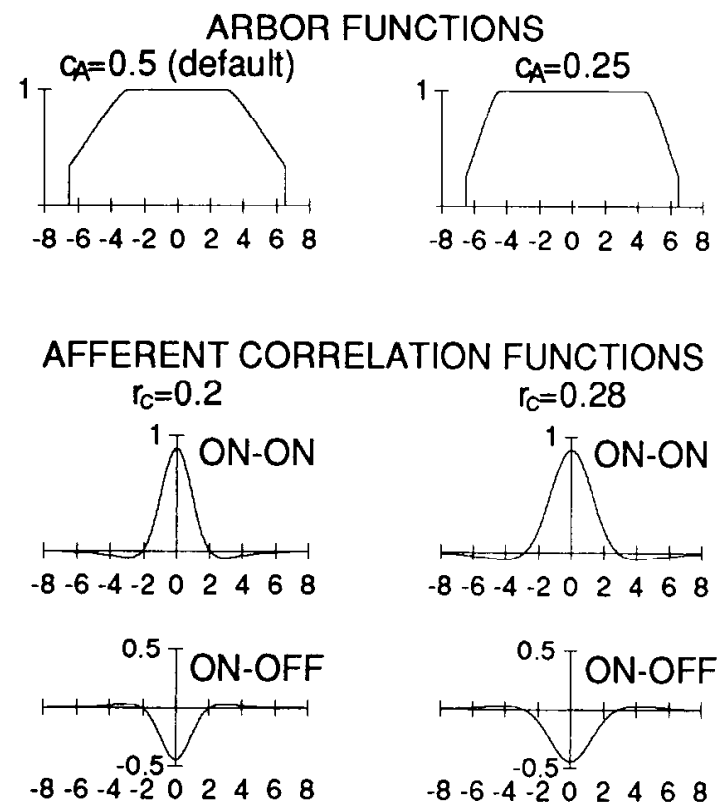

CORTICAL INTERACTION FUNCTIONS
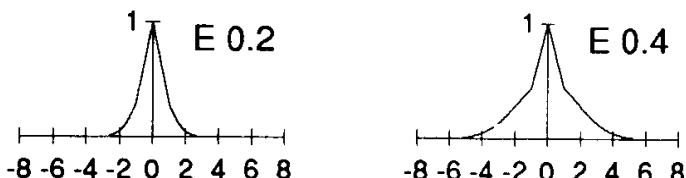

$-8-6-4-2002466$

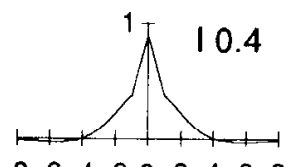

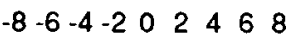

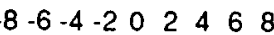

Figure 5. Examples of functions used in simulations. Top, Arbor functions $A(x-\alpha)$ for arbor diameter 13: left, default $\left(c_{4}=0.5\right)$; right, alternative $\left(c_{1}=0.25\right) ; c$, is defined in Methods. Middle, Afferent correlation functions $C^{\mathrm{ONON}}(\alpha-\beta)($ left $)$ and $C^{\mathrm{ONOFF}}(\alpha-\beta)($ right $)$ for arbor diameter 13. $r_{\mathrm{C}}$ is the parameter controlling the width of these functions. Bottom, Intracortical interaction functions $I(x-y)$ : first row, purely excitatory ("E") functions; second row, mixed excitatory/inhibitory ("I") functions. The number (e.g., 0.2 in "E 0.2 ") refers to the value of the parameter $r_{l}$ that controls the width of this function. In all graphs, the horizontal axis represents distance $(|x-\alpha|,|\alpha-\beta|$, or $\mid x$ $-y \mid$, respectively) in grid intervals, while the vertical axis represents the value of the function. All are functions of distance; that is, they are rotationally symmetric. In any single simulation, each function remains fixed; different choices of functions are used in different simulations.

determined by the cutoff of its Fourier transform (the spatial frequency at which its Fourier transform becomes negligibly small compared to the value for frequency 0 ); the corresponding wavelength gives an "effective" arbor diameter. The default taper used here primarily has the effect of shrinking the effective arbor diameter to about 11.3 , for $D_{1}=$ 13.

To define the other functions, first define the Gaussian $G(x, \sigma)=$ $\exp \left(-|x|^{2} / \sigma^{2}\right)$. The ON-ON and OFF-OFF correlation functions have a Mexican hat form, given by a difference of Gaussians: $C^{\text {ONON }}(x)=$ $C^{\text {Сггог }}(x)=G\left(x, r_{c} D_{t} / 2\right)-\left(1 / \gamma_{c}^{2}\right) G\left(x, \gamma_{c} r_{c} D_{t} / 2\right)$. This form sets an approximate balance of correlation and anticorrelation. That is, the $C$ values approximately sum to zero: on a continuum, $\int d^{2} x C(x)=0$ for each $C$. The ON-OFF correlation functions are defined as $C^{\text {ONOFF }}(x)=$ $C^{O F F O N}(x)=-0.5 C^{O N O N}(x)$. This definition is used for simplicity: the

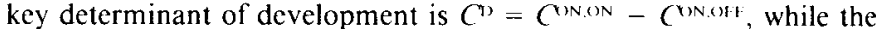
separate values of $C^{\mathrm{ONON}}$ and $C^{\mathrm{ONOHF}}$ are relatively unimportant. The correlation functions are determined by two parameters. $r$, controls the 
width of the Gaussians as a percentage of the arbor radius. It has no default value; it is one of two parameters in the model that are systematically varied. $\gamma_{\varsigma}$ controls the width of the inhibitory Gaussian relative to the excitatory. It has default value $\gamma_{c}=3$. Only $r_{c}$ is systematically varied because the effect of these functions is controlled by a single parameter, the peak of their Fourier transform. As will be shown in Results (Fig. 10), variation of $\gamma_{c}$ has essentially no effect provided $r_{c}$ is covaried to keep this peak constant.

There are two types of intracortical interaction functions. Purely excitatory functions are defined as $I(x)=a(x) G\left(x, 6.5 r_{1}\right)$. Mexican hat functions that mix short-range excitation and longer-range inhibition are $I(x)=a(x)\left[G\left(x, 6.5 r_{l}\right)-\left(1 / \gamma_{t}\right) G\left(x, 6.5 \gamma_{l} r_{t}\right)\right]$. For arbor diameter 13. the function in brackets is identical to the correlation functions,

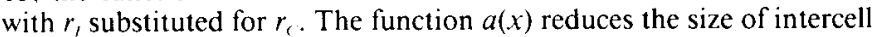
interactions relative to interactions between synapses on the same postsynaptic cell. It is defined by $a(0)=1 ; a(x)=a_{t} \leq 1, x \neq 0$. As with the correlation functions, the effect of $I(x)$ is controlled by the peak of its Fourier transform. Varying $\gamma_{l}$ (default value $\gamma_{,}=3$ ) has essentially no effect provided $r$, is appropriately covaried to keep this peak invariant (see Results, Fig. 10). Altering $a_{1}$ (default value $a_{t}=0.5$ ) does not alter this peak and, as noted in Results, setting $a_{1}-1$ does not visibly alter results. The parameter $r_{t}$, like $r_{c}$, has no default value and is systematically varied. For shorthand, the excitatory $I(x)$ are referred to as " $E$ $r_{i}$ " (e.g., E 0.3, for $r_{t}=0.3$ ), and the mixed excitatory/inhibitory as "I $r_{,}$(e.g., I 0.3 , for $r_{1}=0.3$ ).

Unlike the correlation functions, $I(x)$ does not scale with the arbor diameter. The reasons for this will become more clear from the Results. In essence, this ensures that simulations with given values of $r_{\mathrm{c}}$ and $r_{\mathrm{r}}$ yield similar results across arbor sizes: a given value of $r_{c}$. results in a constant mean number of subregions per receptive field, and thus a roughly constant mean orientation selectivity, while a given value of $r_{r}$ generally leads to a roughly constant width of orientation hypercolumns.

\section{The simulation algorithm}

For brevity, the simulation algorithm is described only for ON-center synapses; it is identical for OFF-center if "ON" and "OFF" are exchanged in all formulas. Simulations were stopped once more than $90 \%$ of synaptic strengths reached their limiting values of 0 or $S_{\max } A(x-\alpha)$. Here $s_{\max }$ is the maximum strength of an individual synapse, with default value $S_{\max }=4$. Synaptic strengths $S^{\mathrm{NN}}(x, \alpha)$ were initially assigned a random strength uniformly distributed over $\left(1 \pm s_{\text {noiss }}\right) A(x-\alpha)$, with $s_{s}=0.2$. The random number generator was ranl from Press et al. (1988). The initial synaptic strengths were multiplicatively normalized over each cortical cell as described in step 5 below. Thereafter, at each timestep, all synapses were updated as follows:

(1) Compute unconstrained derivative $\left.\Delta^{\mathrm{ON}}(x, \alpha) \equiv \frac{d}{d t}\right|_{1,} S^{\mathrm{ON}}(x, \alpha)$ of each synapse $S^{\text {ON }}(x, \alpha)$ from Equation 1. Derivatives are computed by first using the fast Fourier transform to calculate the 4-D convolutions of the form $\Sigma_{1,3} I(x-y) C(\alpha-\beta) S(y, \beta)$ (note that $x, \alpha, y$, and $\beta$ are each 2-D variables). The appropriate sum of convolution terms is then multiplied by the arbor function to yield $\Delta^{\mathrm{oN}}$. The fast Fourier transform is used for speed; the implementation was tested to verify identity of results with the slower, direct calculation. The growth constant $\lambda(\mathrm{Eq}$. 1) was chosen after the first iteration to make the standard deviation of change in synaptic weights for that iteration $\sigma_{\perp}=0.01$. As an extra safety factor, if the result was that $\lambda>\lambda_{10}$, where $\lambda_{0}=0.01,0.02$, respeclively, for arbor diameter 13,11 , then $\lambda$ was set to $\max \left(\lambda / 2, \lambda_{0}\right)$. The value $\sigma_{\lrcorner}$ $=0.01$ was chosen after preliminary simulations in which, for a number of parameter values, no visible synaptic changes occurred as $\sigma_{\lrcorner}$was varied from 0.001 to 0.01 ; at most a few changes in a few synapses occurred as $\sigma$, was increased further to 0.04 ; and only slight changes were visible with further increase through values as large as $0.1 . \lambda_{0}$ was chosen empirically to prevent the number of timesteps to completion from falling below about 40 . Resulting $\lambda$ values ranged from 0.0011 to 0.0377 , with number of timesteps ranging from 36 to 90 .

(2) Modify derivatives with subtractive constraints that conserve total synaptic strength over each cortical cell. The $\mathrm{ON}$-derivative is modified to be $\Delta^{\prime O N}(x, \alpha)=\Delta^{\mathrm{NN}}(x, \alpha)-\epsilon(x) A(x-\alpha)$ where $\epsilon(x)$ is as defined in Equation 3

(3) Use these derivatives plus derivatives from previous timesteps to compute total change in each synapse using a three-step method (Birkhoff and Rota, 1978, p 221), and update synaptic strengths. The threestep method allows larger timesteps (larger $\lambda$ ) and thus faster computation. The three-step method used for integrating $\frac{a}{d i} \mathbf{S}(t)=\lambda \mathbf{F}(\mathbf{S})$ was
$\mathbf{S}(t+1)=\mathbf{S}(t)+\lambda(23 \mathbf{F},-16 \mathbf{F},+5 \mathbf{F},) / 12$, where $\mathbf{F}$, is the value of $\mathbf{F}(\mathbf{S})$ at time $t$; here, $\mathbf{F}$, is the final derivative after step 2 at time $t$. The first two updates were $\mathbf{S}(t=1)=\mathbf{S}(t=0)+\lambda \mathbf{F}_{0} ; \mathbf{S}(t=2)=\mathbf{S}(t=$ 1) $+\lambda\left(2 \mathbf{F}_{1}-\mathbf{F}_{t)}\right)$. After computation of $\mathbf{S}(t=4)$, the step size and $\lambda$ were doubled, so that thereafter only even-numbered timesteps were computed, as $\mathbf{S}(t+2)=\mathbf{S}(t)+2 \lambda\left(23 \mathbf{F}_{t}-16 \mathbf{F}_{t_{2}}+5 \mathbf{F}_{t_{4}}\right) / 12$.

(4) If $S^{(N)}(x, \alpha)<0$ or $S^{\prime N}(x, \alpha)>s_{\max } A(x \quad \alpha)$, cut off value at 0 or $S_{\text {mix }} A(x-\alpha)$, respectively; similarly for $S^{\text {orf }}(x, \alpha)$.

(5) If any synapses are cut off in step 4 , correct for this by multiplicatively renormalizing the synaptic strength over the corresponding cortical cells. Each synaptic strength on the cortical cell at $x$ is multiplied by $\gamma(x)=\left[2 \Sigma_{i s} A(x-\beta)\right] /\left(\Sigma_{i}\left[S^{\circ \mathrm{N}}(x, \beta)+S^{\circ H}(x, \beta)\right]\right)$. This restores total synaptic strength on that cell to $2 \Sigma_{3} A(x-\beta)$, as it was before step 4 .

Saturated synapses were stabilized; that is, if synaptic strengths reached the limiting values of 0.0 or $s_{m i x} A(x-\alpha)$, they were frozen so that no further changes in their strengths were allowed. This is done for computational convenience and does not alter results (Miller, 1989a; Miller et al., 1989). To implement stabilization, frozen synapses were assigned derivatives $\Delta=0$, steps 2 and 5 were applied only to active (unfrozen) synapses, and the formulas for $\epsilon(x)$ in step 2 and $\gamma(x)$ in step 5 were modified as follows. Let $S_{\text {aci }}(x)$ be the summed strength of all active synapses (both ON- and OFF-center) on the cortical cell at $x$, and let $S_{\mathrm{frro}}(x)$ be the summed strength of the frozen synapses. Let $A_{\mathrm{act}}^{\mathrm{ON}}(x, \alpha)=$ $A(x-\alpha)$ if $S^{\mathrm{NN}}(x, \alpha)$ is active, $=0$ if $S^{\mathrm{NN}}(x, \alpha)$ is frozen [and similarly for $\left.A_{\mathrm{acl}}^{\mathrm{OFF}}(x, \alpha)\right]$. Then $\mathrm{\epsilon}(x)=\left(\Sigma_{j}\left[\Delta^{\mathrm{ON}}(x, \beta)+\Delta^{\mathrm{OFF}}(x, \beta)\right]\right) /\left(\Sigma_{i s}\left[A_{\mathrm{acl}}^{\mathrm{ON}}(x, \beta)\right.\right.$ $\left.\left.+A_{\mathrm{act}}^{\mathrm{OCF}}(x, \beta)\right]\right)$; and $\gamma(x)=\left[-S_{\text {fro }}(x)+2 \Sigma_{i j} A(x-\beta)\right] / S_{\mathrm{acl}}(x)$, subject to $0.8 \leq \gamma(x) \leq 1.2$.

\section{Calculation of properties of receptive fields}

Responses of model cortical cells to sine wave gratings of all orientations and spatial frequencies were calculated under a simple linear model of response. Define the ON/OFF difference: $S^{\mathrm{D}}(x, \alpha) \equiv S^{\mathrm{ON}}(x, \alpha)-S^{\mathrm{OFF}}(x$, $\alpha)$. Response is defined as the product of the grating with the receptive field pattern of $S^{\mathrm{D}}$. Let $\mathcal{R}(x, k, \phi)$ be the response of the cell at $x$ to the grating $\sin ([2 \pi k \cdot(x-\alpha) / 64]+\phi)$ (note that $k$, like $x$ and $\alpha$, is a 2-D vector with integer components). Then $\mathcal{R}(x, k, \phi)=\Sigma_{a x} S^{\top}(x, \alpha) \sin ([2 \pi k$. $(x-\alpha) / 64]+\phi)$. It suffices to know $\mathcal{R}(x, k)=\max _{\phi} \mathcal{R}(x, k, \phi)$, which can be calculated directly: $R(x, k)=\left|\hat{S}^{1}(x, k)\right|$, where $\tilde{S}^{1}(x, k)$ is the Fourier transform over $\alpha$ of $S^{\mathrm{D}}(x, \alpha)$, on a 64 by 64 grid whose edges are zero-filled.

The preferred spatial frequency and, in some cases, preferred orientation of the receptive field were assigned as the spatial frequency and orientation, respectively, of the sine wave grating giving maximal response. This definition of preferred spatial frequency is in accord with experiment (Movshon et al., 1978a; see further discussion below). To assess the degree of orientation selectivity, the best response over all gratings (all wavenumbers $k$ ) in each $10^{\circ}$ interval of orientation between $0^{\circ}$ and $180^{\circ}$ was determined. This defines a function $R(x, n)$, given by the best response of the cell at $x$ to gratings in the $n$th orientation interval, $n=0, \ldots, 17$. For the $n$th interval, a $2-\mathrm{D}$ vector was constructed with length $R(x, n)$ and angle $n \times 20^{\circ}$. The vector sum of these 18 vectors gives a vector $v_{R}(x)$, with length $\left|v_{R}(x)\right|$. The orientation selectivity $O(x)$ is defined as the ratio of $\left|v_{R}(x)\right| / 18$ to the rms length of the 18 individual vectors. Selectivity, so defined, takes values from 0 to 1 .

Because the measure of response is linear and receptive fields are finite in size, even a set of weights corresponding perfectly to a sine wave grating gives positive responses at almost all orientations. For this reason, it is impossible for this response model to achieve biological levels of selectivity. In practice, the maximum selectivity is about 0.28 for model receptive fields. Nonetheless, this response model is sufficient so that the measure of selectivity correlates well with the degree of tuning expected based on observation of the spatial structure of the receptive field. This is illustrated in Figure 6, which shows receptive fields for selectivities ranging from 0 to 0.27 . Orientation tuning curves under this linear response model are also shown. Note that, for receptive fields like these with oriented, segregated sets of $\mathrm{ON}$ - and OFF-center inputs, a more realistic response model can yield sharp orientation tuning curves with physiological levels of selectivity (Ferster, 1987).

A cell's preferred orientation can also be defined as the orientation corresponding to $v_{k}(x)$ [i.e., the angle of $v_{R}(x)$, divided by 2]. This and the previous definition give results that are not disccrnibly different. The definition based on $v_{R}(x)$ was used in maps that simultaneously display the preferred orientation and the degree of orientation selectivity.

Mathematically, the orientation selectivity $O(x)$ can be expressed in 
Figure 6. Assessment of orientation selectivity. Receptive fields and orientation tuning curves of cells with steadily decreasing assessed values of orientation selectivity are shown from two simulations. Selectivity is the number written above each receptive field or orientation tuning curve. $A$, Cells with narrower $\mathrm{ON}$ - and OFF-regions, resulting from development with a narrow correlation function, $r_{c}=0.2 . B$, Cells with broader ON- and OFFregions, resulting from development with a broader correlation function, $r_{\mathrm{C}}$. $=0.28$. Histograms show best "response" (as defined in Methods) to any sine-wave grating, across all spatial frequencies and phases, in each $10^{\circ}$ interval of orientation $0-10^{\circ}, \ldots, 170-180^{\circ}$; bin to the side shows response to uniform luminance. Note that the linear "response" measure used is not meant to achieve biological sharpness of tuning (see Methods). Receptive fields show difference between ON-center and OFFcenter synaptic strength from each input position; conventions and gray scale are as described for Figure 7.
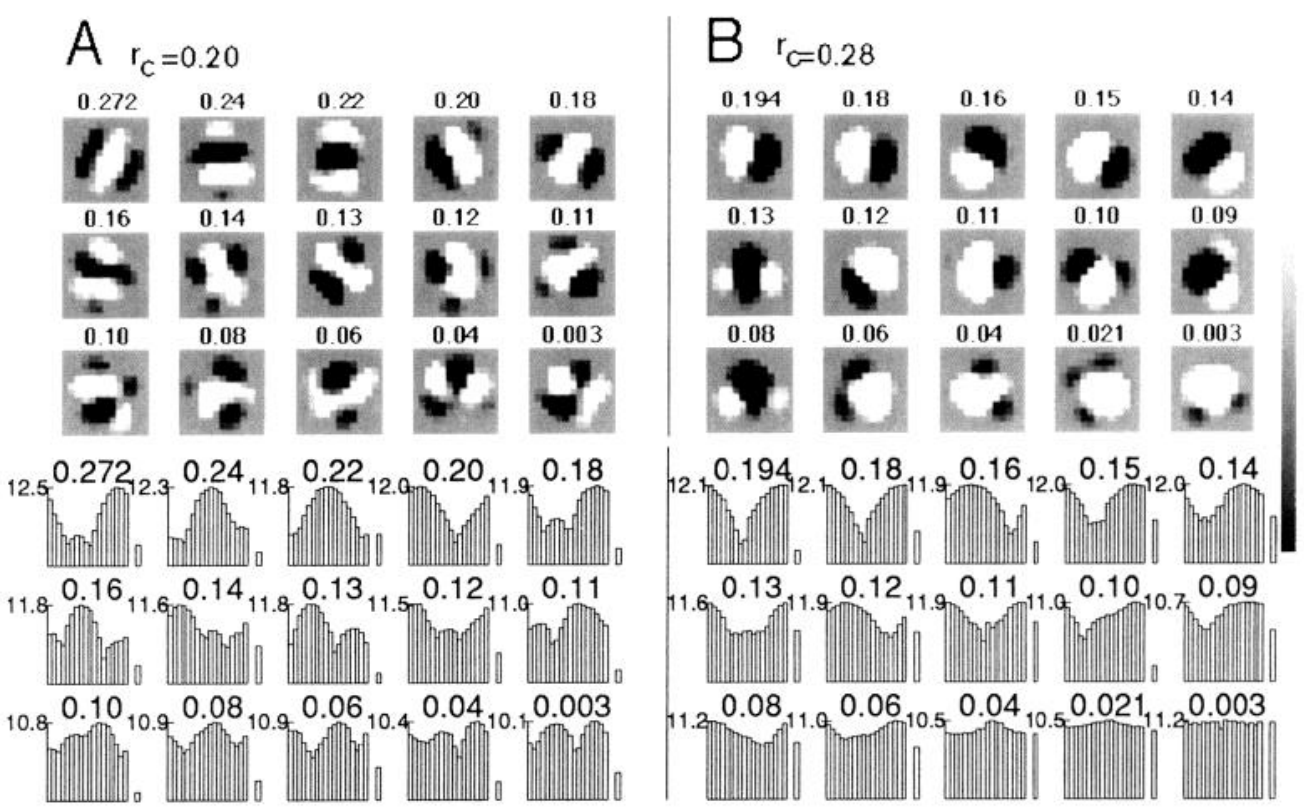

terms of $\tilde{R}(x, p)$, the Fourier transform of the orientation tuning curve: $\tilde{R}(x, p) \equiv \Sigma_{n=0}^{N_{*}-1} R(x, n) \exp \left(2 \pi i p n / N_{o}\right)$, where $N_{o}$ is the number of orientation bins (here, $N_{o}=18$ ). Then $O(x)=|\tilde{R}(x, 1)| / \sqrt{\Sigma_{n}|\tilde{R}(x, p)|^{2}}$. Omitting the $x$ index: use of $\tilde{R}(1)$ to characterize selectivity was proposed in Wörgötter et al. (1990), but with the normalization $O_{w}=|R(1)|$ / $|\tilde{R}(0)|$ [they refer to $\tilde{R}(1)$ as the second rather than first harmonic, because they define orientation over $360^{\circ}$ rather than $180^{\circ}$ ]. $O_{w}$ is the ratio of $\left|v_{R}\right| / N_{o}$ to the mean, rather than rms, length of the $N_{o}$ individual vectors that define $v_{R}$. $O_{w}$. may take values larger than 1 when some responses are negative. A normalization that avoids this is (Chapman, 1991) $O_{C}=O_{u} /\left(1+O_{u}\right)$. The difference between the various measures is their treatment of higher harmonics. With the linear definition of response used here, higher harmonics primarily represent multiple peaks in the response function, so their presence should decrease the assessment of orientation selectivity; hence, the present normalization is preferred. For physiological data, multiple peaks in the response function are rarely found (but see Wörgötter and Eysel, 1991a) and higher harmonics primarily represent "sharp edges" in the response function (e.g., a cell that shows positive response to some orientations and no response to the others) (B. Chapman, personal communication); in this case, the normalizations of $O_{w}$ or $O_{C}$ are preferred.

\section{The meaning of a "receptive field"}

The measure of cortical cell response defined above, $\mathcal{R}(x, k, \phi)$, takes into account only the set of LGN synaptic weights received by the cortical cell. This ignores two other factors that also contribute to cortical visual responses, namely, (1) intracortical synapses and (2) the receptive fields of the LGN afferents. More generally, in considering model results I use the term "receptive field" to describe the pattern of LGN synaptic weights received by a cortical cell, whereas physiologically, "receptive field" describes the response of a cell to visual stimulation and thus would incorporate all of these factors.

One justification for these simplifications is that the spatial pattern of LGN synaptic weights should be adequate to predict general properties of simple cell responses such as preferred orientation and preferred spatial frequency. The basis for this statement is twofold. (1) These response properties depend only on the spatial pattern of the $\mathrm{ON}$ - and OFF-subregions in the simple cell's receptive field (as physiologically measured). Many reports, beginning with Hubel and Wiesel (1962), have established that a simple cell's preferred orientation is well predicted by the direction of the linear arrangement of the cell's ON- and OFFsubregions. Similarly, Movshon et al. (1978a) showed that the spatial frequency response of a cat simple cell is well predicted from the spatial structure of the ON- and OFF-subregions of the receptive field. (2) The spatial pattern of the ON- and OFF-subregions in the cortical cell's receptive field (as measured physiologically) should, in turn, be reasonably well predicted by the corresponding pattern in the LGN weights received by the cell, assuming that these LGN weights form cleanly segregated ON- and OFF-subregions as in the present model cells.

The effects of LGN receptive fields and intracortical interactions on cortical responses were directly studied as follows: "physiological" weights $P^{\mathrm{ON}}(x, \alpha)$ were defined by $P^{\mathrm{ON}}(x, \alpha)=\Sigma_{\text {v: }} I(x-y) S^{\mathrm{ON}}(y, \beta) L(\beta-\alpha)$ (and similarly for POFF), where $L(\beta-\alpha)$ is an LGN receptive field center profile. The major effect, relative to the receptive fields formed by the LGN weights $S^{\mathrm{ON}}(x, \alpha)$, was to enlarge slightly the receptive fields, by elongating subregions, widening subregions at the receptive field edges, or adding small additional subregions at these edges. This induced small changes in preferred spatial frequencies (mean change, increase of 4 $5 \%$, and rms change, $10-15 \%$, for two simulations studied). The orientation maps were largely unchanged but became slightly smoother; Fourier transforms of these maps were virtually unchanged but showed a slight shift of power to longer frequencies.

This definition of physiological weights involves a number of arbitrary assumptions about cortical integration and so does not necessarily produce receptive fields more biologically based than the geniculate weights themselves. Hence, it was judged preferable to present the geniculate weights, which constitute the "raw data" produced by the model, and to focus on general response properties, such as preferred orientation and preferred spatial frequency, that should not significantly depend on the model of cortical integration.

\section{Analysis of cortical maps}

To determine the spatial period with which orientation varies across cortex (i.e., the width across cortex of a complete cycle of orientations), the power spectra of model cortical maps of orientation were determined. To do so, the function $v_{R}(x)$ was Fourier transformed on the 32 by 32 cortical grid. The 2 -D vector $v_{R}(x)$, regarded as a complex number, is proportional to the first harmonic of the response function, $\tilde{R}(x, 1)$. Its Fourier transform is $\hat{R}(k, 1)=\Sigma_{x} \exp (2 \pi i k \cdot x / 32) \tilde{R}(x, 1)$. The power $P(k)$ at each 2-D wavenumber $k=\left(k_{1}, k_{2}\right),-16 \leq k_{1}, k_{2} \leq 15$, is $P(k)$ $=|\hat{R}(k, 1)|^{2}$. Power at wavenumber $k$ represents a periodic arrangement of orientations with a complete cycle of orientations in $32 /|k|$ grid intervals, where $|k| \equiv \sqrt{k_{1}{ }^{2}+k_{2}{ }^{2}}$.

The 1-D power spectrum, $p(s)=\Sigma_{|k|-s} P(k)$, gives a measure of the width of orientation hypercolumns: the power at $s$ represents the degree to which orientation hypercolumns (complete cycles of $180^{\circ}$ of orientation) have width $32 / s$. To compute $p(s)$, the 2-D spectrum was divided into annuli as follows: an inner circle had radius 0.23 (in $|k|$ ); subse- 
quent annuli had increments of radius 0.4 . These numbers were chosen empirically as those which left few empty annuli. Let $s$ be the midpoint $|k|$ of the $i$ th annulus, and $\tilde{p}\left(s_{i}\right)$ the summed power in that annulus. Then $p\left(s_{i}\right)$ was computed as $\left.p\left(s_{i}\right)-\left[\hat{p}\left(s_{i}\right)+2 \hat{p}\left(s_{i}\right)+\hat{p}\left(s_{i}\right)\right)\right] / 4$ (at the two edges, the nonexistent annulus was omitted and the division was by 3 ).

Power spectra in Figure 15 of linearly interpolated model maps were determined similarly to other model spectra, except that (1) these maps were periodic on, and Fourier transformed on, a 128 by 128 rather than 32 by 32 grid; (2) only the preferred orientation $\theta(x)$ was interpolated, so $v_{R}(x)$ was assigned magnitude 1 and direction $2 \theta(x)$.

Power spectra of experimental maps of orientation were determined as follows. These maps were either 192 by 144 or 128 by 128 pixels. The vectors $b_{k}(x)$ were provided; they had been determined by assessing the response at eight orientations and adding these responses as vectors, as in the definition of $v_{k}(x)$ above. The central 144 by 144 or, for smaller maps, 128 by 128 square of the map was placed on a 256 by 256 grid, with the remainder of the grid filled with zeros. Power was then determined by Fourier transform, with $v_{R}(x)$ regarded as a complex number as above. To convert this $2-\mathrm{D}$ power spectrum to a $1-\mathrm{D}$ spectrum, the central 32 by 32 portion of the $2-\mathrm{D}$ spectrum was analyzed as just described for model cortices; power outside this central portion was negligible.

Vortices or singularities of an orientation map were determined as follows. For each pixel in the map, a clockwise path is taken around the four neighboring pixels that form a square "down and to the right" (using periodic boundary conditions). Let the preferred orientation at these four pixels be $\theta_{i}, i=0$ through $3,0 \leq \theta_{1}<180$, where $i$ is numbered sequentially around the path, and let $\theta_{4}=\theta_{41}$. The index of the path is defined to be $\Sigma^{3}, \phi\left(\theta_{i, 1}-\theta_{t}\right) / 360^{\circ}$, where $\phi(x)=x,|x| \leq 90^{\circ} ; \phi(x)=$ $x-180^{\circ}, x>90^{\circ} ; \phi(x)=x+180^{\circ}, x<-90^{\circ}$. The index is equal to $N / 2$ for an integer $N$. If $N$ is nonzcro, the path encloses a vortex around which orientation cycles $N$ times. If $N$ is positive, the orientation increases (cycles counterclockwise) for clockwise movement around a path, while negative $N$ denotes decrease for clockwise movement. Vortices add in the sense that a simple closed path through the orientation map traverses $180 N_{t o n}$ degrees, where $N_{t o r} / 2$ is the sum of the indices of the vortices enclosed by the path.

The gradient of an orientation map (rate of change of preferred orientation across cortex) was computed as follows. The difference between the preferred orientations $\theta_{1}, \theta_{2}$ of two cells is defined as $\Delta\left(\theta_{1}-\theta_{2}\right)=$ $\phi\left(\left|\theta_{1}-\theta_{2}\right|\right)$, with $\phi$ as just defined. At each pixel, the horizontal component of the gradient $G_{n}$ is taken to be the mean of the $\lrcorner$ values with the two horizontal nearest neighbors, the vertical component $G_{1}$ the mean of the $\Delta$ values with the two vertical nearest neighbors. The (scalar) gradicnt is defincd as $G=\sqrt{G_{h}{ }^{\prime} / \bar{G}_{\mathrm{r}}}$. This represents approximately the change in degrees of orientation for a movement of one grid interval in the fastest-changing direction.

\section{Prediction of mean preferred spatial frequencl of cortical simple cells}

As discussed in Results, the mean preferred spatial frequency of simple cells is predicted to be $1 /(2 L)$, where $L$ is the diameter of the positive central region of the difference correlation function $C^{\prime \prime}$; and I approximate $L$ for inputs to cat area 17 or area 18 by twice the separation over which adult cat retinal X-cell or Y-cell correlations, respectively, go to zero (Mastronarde, 1983a,b). Then spatial frequencies are predicted as follows: for X-cells, adult retinal correlations go to zero at a separation of one dendritic field diameter. X-cell dendritic field diameter varies from 3.6 retinal ganglion cell (RGC) spacings at $2^{\circ}$ eccentricity, to 4.4 RGC spacings at $12^{\circ}$ eccentricity (Mastronarde, 1983a, p 306) Values at other eccentricities were obtained by linear interpolation or extrapolation from these two values. To convert RGC spacings to degrecs of visual angle, the following formula was used (Mastronarde, 1983a): let $S=$ RGC spacings per degree of visual angle, and $D=$ density of retinal ganglion cells in cells per $\mathrm{mm}^{2}$; then $S=0.186 \sqrt{D}$. Implementation of this conversion was based on a program provided to me by $D$. N. Mastronarde, which included values of $D$ as a function of eccentricity and polar angle. A weighted average of $S$ was computed, weighted by cell density (since higher density means more inputs to cortex). The linear interpolation and the weighting have very little effect on the final curve: the primary contributing factors are the variation of $S$ with eccentricity, and the value of about 4 RGC spacings for the radius over which correlations go to zcro.
For Y-cells, Mastronarde's data are more variable. A cutoff of 14 RGC spacings is suggested most strongly by the data (based on Mastronarde, 1983a, Figs. 3.4. 5.1), and was used, but values between 12 and 20 might also be justified. A smaller value would move the $Y$-cell curve vertically up; a larger value, vertically down. Y-cell dendritic diameters range from 23.3 RGC spacings at $2^{\circ}$ eccentricity to $17.8 \mathrm{RGC}$ spacings at $12^{\circ}$ eccentricity (Mastronarde, 1983a, p 306). I assumed that the correlations scale with this diameter, and linearly interpolated, proportionally to these two values, a line that passes through 14 RGC spacings at $7^{\circ}$ eccentricity (the eccentricity at which X-cells have the value 4 RGC spacings). The procedure was otherwise identical to that for the X-cell curve.

\section{Results}

The development of synaptic connectivity has been studied through both computer simulations and mathematical analysis. The purpose is to characterize in a general way the determinants of development under the proposed hypothesis. Our knowledge of the relevant biological parameters during development is at best crude, as it is generally inferred from circumstantial and indirect evidence based on studies in mature animals. Therefore, we wish to understand the range of outcomes that may occur and the conditions under which they occur. In this way, we may determine whether the hypothesis can robustly account for biological results across a reasonable range of parameters.

\section{Typical outcomes of simulations}

A typical development with a Mexican hat correlation function, for a case in which intracortical connections are purely excitatory $\left(\mathrm{E} 0.3, r_{c}=0.24\right)$, is illustrated in Figure 7 . The receptive ficlds of a 5 by 5 patch of cortical cells initially receive a patchy mix of both ON-center and OFF-center inputs (Fig. 7.4). Each cell gradually develops an oriented receptive field with distinct, cleanly segregated ON-subregions (white) and OFF-subregions (hlack). Across the cortex, cells are initially randomly and poorly oriented (Fig. $7 \mathrm{~B}$ ); hue indicates the preferred orientation of a cell, while brightness indicates the degree of orientation selectivity. The cells gradually develop a continuous and periodic arrangement of preferred orientations that qualitatively resembles that observed experimentally (Blasdel and Salama, 1986).

The types of receptive fields and cortical organization that typically emerge are illustrated in Figure 8. Final results are presented from four simulations that began with identical initial synaptic weights but developed under differing parameters. The simulations used two widths of correlation function (narrower, $r_{1}=0.24$, or broader, $r_{c}=0.28$ ) and two types of intracortical interaction (excitatory, E 0.3 , or mixed excitatory/inhibitory, I 0.3 ). Figure 8.4 presents the full 32 by 32 sets of mature cortical receptive ficlds from the $r_{\mathrm{c}}=0.28$ simulations, and 18 by 32 receptive fields from the $r_{c}=0.24$ simulations (corresponding to the rightmost 18 columns in the $r_{c}=0.28$ cortices). The broader correlation function results in fewer ON/OFF subregions per receptive field than the narrower function. Despite this change in receptive field structure, preferred orientations remain largely unchanged, on a cell-by-cell basis, as the correlation function is changed (compare corresponding cells in the two E 0.3 cortices, or the two I 0.3 cortices). Preferred orientations are altered when the intracortical interaction function is changed: the mixed excitatory/inhibitory intracortical interaction function leads to a more rapid change of preferred orientation than the purely excitatory. The swirling, continuous changes of preferred orientation that emerge are particularly visible in the more slowly varying, E 0.3 cortices.

In all four cases, a majority of receptive fields become ori- 

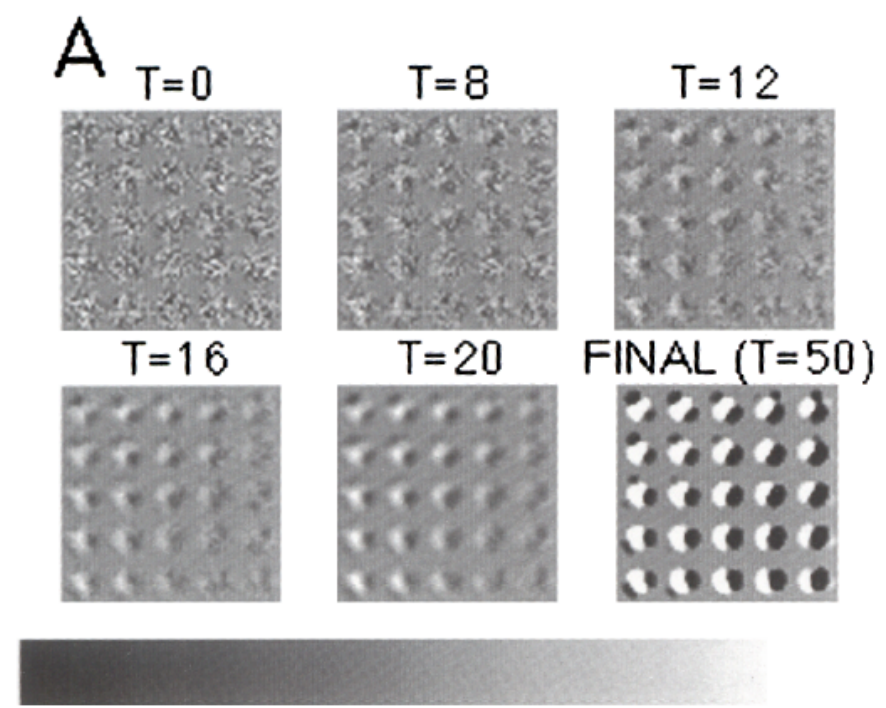

OFF

ON

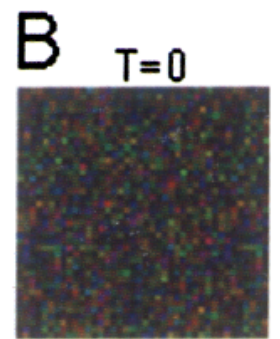

$T=16$
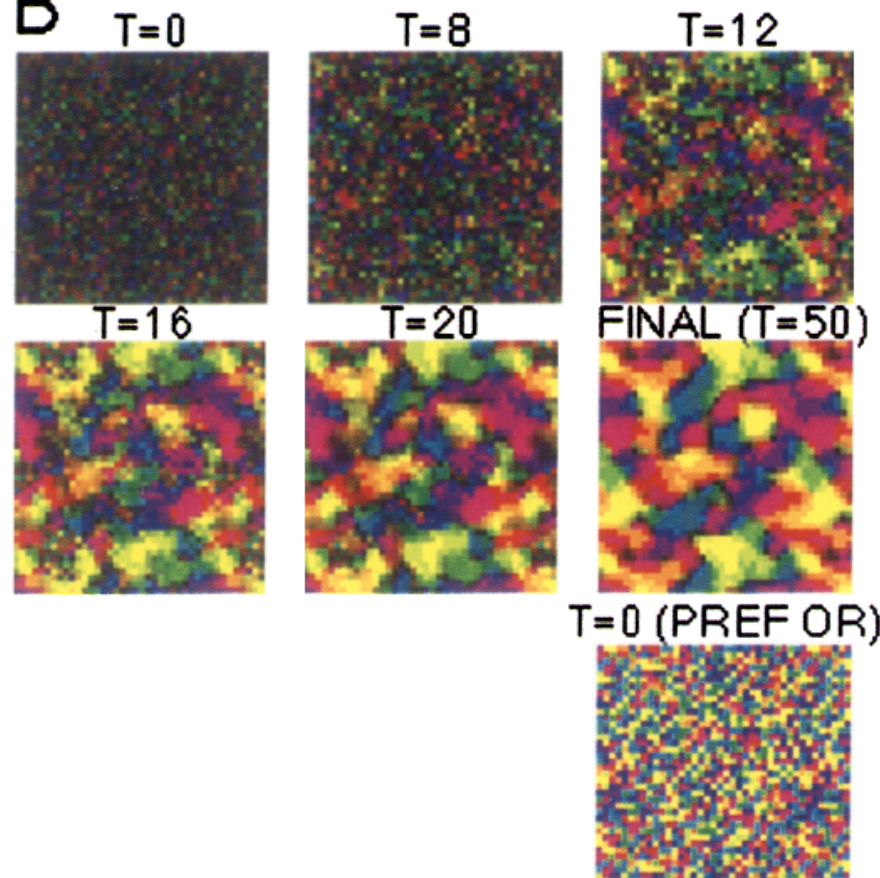

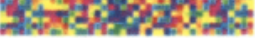

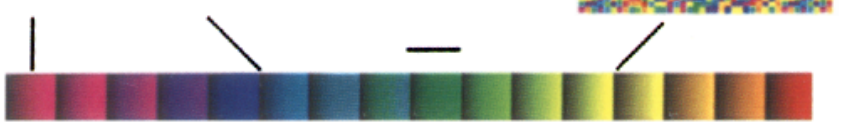

Figure 7. Development of orientation selectivity. Shown are illustrations of results from a single simulation, using the purely excitatory intracortical interaction function E 0.3 , and the afferent correlation functions with $r_{C}=0.24$ (see Fig. 5). $A$, Development of the receptive fields of a 5 by 5 patch of cortical cells. Difference between ON-center and OFF-center synaptic strength at each point in each receptive field is shown, at six developmental times ranging from a randomly assigned initial condition $(T=0)$ to the final state ( $T$ is the number of iterations). White represents $\mathrm{ON}$-dominance; black, OFF-dominance; gray, equality (see gray scale at bottom). At each time, the 5 by 5 receptive fields are shown adjacent to one another without intervening spaces; that is, the large square shown at a single time consists of 5 by 5 adjacent subsquares, where each subsquare shows the 13 by 13 set of inputs to one cortical cell. Corners of each receptive field are always zero (gray), because connectivity is restricted to a circle of diameter 13 . $B$, Development of the overall cortical pattern of preferred orientation and ori- entation selective (60-67\% of cells in each of these simulations have selectivity $\geq 0.12$, which represents good selectivity as was shown in Fig. 6; see also Fig. 9C), and there is continuous organization of preferred orientation across the cortex. However, some cells do not become orientation selective. These poorly oriented cells primarily occur at regions of high "orientation gradient," that is, where preferred orientation changes rapidly with cortical position (see Fig. $8 B, C$ ). Such cells are at a boundary between two or more regions of cells with different preferred orientations, and hence develop receptive fields that "compromise" between these differing neighbors. Factors present biologically but absent from the model, such as plasticity of intracortical synapses or nonlinearities in activation and plasticity rules, might lead such boundary cells to develop a single preferred orientation rather than a linear "mix" of orientations as here.

Systematic organization across cortex of the spatial phase of receptive fields is evident in Figure $8 A$. Here, "spatial phase" refers to the location within the receptive field of the ON-subregions and the OFF-subregions (more precisely, it refers to the spatial phase of the sine wave to which the receptive field gives best response; see Methods). In the E 0.3 cortices, clusters of cells with similar preferred orientations show regular shifts of spatial phase with movement across cortex perpendicular to the preferred orientation (e.g., with horizontal movement across cells preferring vertical orientation). Some examples are outlined with white dashed lines. The cells remain largely invariant in spatial phase with movement across cortex along the preferred orientation. Each receptive field is shifted by one grid position in retinotopic position from its neighbors, so these spatial phase relationships roughly align ON-subregions retinotopically with ON-subregions and OFF-subregions with OFFsubregions among neighboring cortical cells. This leads neighboring cells to be correlated, consistent with the purely excitatory E 0.3 intracortical interaction. The mixed excitatory/inhibitory

entation selectivity. Preferred orientations are represented by hue, and in the top six panels the degree of orientation selectivity is represented by brightness (see color scale at bottom). The bottom right panel shows preferred orientation at $T=0$ without regard to selectivity: the final pattern of preferred orientations is organized by the competitive mechanism and does not stem from any prepattern. Conventions for this and other figures: 5 by 5 receptive fields are those of the bottom left 5 by 5 square in the corresponding orientation map. At later times and in other figures, gray regions interior to the receptive fields represent weak synaptic strengths, rather than overlap of strong but equal ON- and OFFcenter innervations (the two would be indistinguishable since only the difference between ON and OFF innervation is shown). Images at each timestep have been scaled so that white and black represent, respectively, the maximum and the minimum difference $D$ between ON- and OFFcenter strengths among illustrated receptive fields at the given time. Maximum difference $D$ and maximum synaptic strength $S$ among receptive fields shown are T $=0, D=0.39, S=1.21 ; \mathrm{T}=8, D=0.53$, $S=1.48 ; \mathrm{T}=12, D=0.80, S=1.77 ; \mathrm{T}=16, D=1.51, S=2.35 ; \mathrm{T}$ $=20, D=3.38, S=3.58 ; \mathrm{T}=50$ and other figures, $D=S=4$. In orientation maps, preferred orientation (hue) ranges linearly in 16 steps from vertical (red, left of color scale), counterclockwise to $11.25^{\circ}$ clockwise from vertical (reddish color at right of color scale), as indicated by oriented lines above color scale. Orientation selectivity (brightness) is displayed, for each hue, on a linear scale in 16 steps of brightness. These correspond, from dimmest to brightest, to selectivities 0-0.01, 0.01$0.02, \ldots, 0.14-0.15,0.15$ and above. To show the continuity across the periodic boundary conditions, the 32 by 32 grid of cortical cells is shown as a 40 by 40 grid, with the leftmost eight columns repeated at the right and the bottommost eight rows repeated at the top. 
I 0.3 interaction leads to more varied combinations of phase changes and orientation changes. In some cases in the I $0.3, r_{C}$ $=0.28$ cortex, the cells in an iso-orientation cluster have nearly identical receptive fields; that is, spatial phase varies slowly or not at all (some examples are outlined with dashed lines). These relationships lead cortical cells to be correlated over excitatory distances and anticorrelated over inhibitory distances, as will be discussed in more detail later.

The orientation maps contain a number of vortices or "pinwheels," points around which a circular path through neighboring cells cycles through $\pm 180^{\circ}$ of preferred orientation (Bonhoeffer and Grinvald, 1991). All such vortices are marked with asterisks in the $\mathrm{E} 0.3, r_{c}=0.28$ cortex of Figure $8 \mathrm{~A}$. With this broader correlation function, some clusters of cells develop poor orientation selectivity and low preferred spatial frequencies [preferred spatial frequency is essentially inversely proportional to the width of the ON- and OFF-subregions of the receptive field (Movshon et al., 1978a); see Methods]. This is reminiscent of the conjunction of poor orientation selectivity and low preferred spatial frequencies reported in cytochrome oxidase blobs in monkey visual cortex (Silverman et al., 1989). While some vortices develop such "blob-like" features, there are also many vortices that are not "blobs" and "blobs" that are not vortices (two such nonvortex "blob-like" regions are marked with "+" in the $\mathrm{E} 0.3, r_{\mathrm{C}}=0.28$ cortex). Such an overlapping but nonidentical relationship is found experimentally between blobs and vortices (Bartfeld and Grinvald, 1992; Blasdel, 1992).

Other receptive field properties become clustered in cortex, with the excitatory intracortical interaction yielding a broader spatial scalc of organization than the mixed excitatory/inhibitory. This is evident in Figure $8 B$, which presents the cortical maps of the degree of orientation selectivity, orientation gradient. preferred spatial frequency, and ON- or OFF-dominance from these four simulations (maps of preferred orientation will be found in Fig. 9B). Orientation gradient is a measure of the rate of change of preferred orientation across cortex; it is high where preferred orientation changes most rapidly. Cells with low orientation selectivity primarily occur in regions of high gradient (Fig. 8B,C). With the broader correlation function, cells with both the very lowest and the very highest preferred spatial frequencies tend to have poor orientation selectivity (Fig. 8C). Particularly with this broader function, a periodic arrangement of ON-dominated and OFF-dominated regions emerges. This is reminiscent of afferent segregation in the cortex of minks (McConnell and LeVay, 1984) and ferrets (Zahs and Stryker, 1988), but segregation in the model is necessarily less complete. Biologically, such afferent segregation may represent either segregation between postsynaptic cells, or convergence onto individual postsynaptic cells with spatial segregation occurring at the level of dendrites. These simulations model the case of convergence, for which only weak segregation can develop because cortical cells are modeled as points without spatially extended dendrites.

\section{Parameter dependence}

These basic results persist across a range of correlation and intracortical interaction functions (Fig. 9). The correlation function is the primary determinant of receptive field structure: broader functions yield wider ON/OFF subregions and thus fewer subregions per receptive field than narrower functions (Fig. 9A). The cortical organization of preferred orientation is most influenced by the intracortical interaction function: narrowing of excitatory intracortical interactions or addition of lateral inhibition yields a narrower spatial period of preferred orientation (Fig. 9B). Across a range of functions, orientation selectivity robustly develops (Fig. $9 C$ ) and is organized into a continuous, periodic cortical map.

Only one other parameter significantly influences the outcome, in addition to those controlling the widths of the functions. This is $S_{\max }$, the maximum synaptic strength, which determines the number of synapses in a final receptive field and thus the final receptive field diameter. There is a constraint conserving total synaptic strength $S_{10 t}$ over each receptive field, and almost all synaptic strengths eventually saturate at 0 or $S_{\text {nax }}$ (see Methods). 'Ihus, the number of final synapses is approximately $S_{10 t} / S_{\text {nax }}$. Increasing $S_{\max }$ from 4 to 8 (Fig. 10.1) results in smallcr receptive ficlds, and in some cases fewer subregions per receptive field or even unimodal cells (cells with only a single

\footnotetext{
Figure 8. Final results from four simulations. Results are shown from the same simulation as in Figure $7\left(\mathrm{E}, 0.3, r_{c}=0.24\right)$ and from three additional simulations beginning with identical initial synaptic strengths but using different parameters $\left(\mathrm{E} 0.3, r_{\mathrm{c}}=0.28 ; \mathrm{I} \quad 0.3, r_{\mathrm{c}}=0.28\right.$ and $r_{\mathrm{c}}$ $=0.24)$. A, Full 32 by 32 set of final receptive fields from $r_{c}=0.28$ simulations, and 18 by 32 set of final receptive fields from $r_{c}=0.24$ simulations (these cells correspond to the rightmost 18 columns of thc $r_{c}=0.28$ simulations). Conventions and gray scale are as in Figure $7 . \mathrm{A}$. Note that, because of periodic boundary conditions, the top and bottom rows in each simulation are neighbors, as are the left and right columns in the $r_{\mathrm{c}}=$ 0.28 cortices. White dashed lines outline examples of regular, progressive phase shifts among cells with movement perpendicular to the preferred orientation (E 0.3, $r_{c}=0.28$ cortex) or of little or no phase shift across a group of cells (I $0.3, r_{c}=0.28$ corlex). In the $\mathrm{E} 0.3, r_{c}=0.28$ cortex, certain special points are marked as follows. All vortices (points around which orientation cycles by $180^{\circ}$; compare corresponding orientation map in Fig. $9 B$ ) are marked by asterisks. Black asterisks indicate index $-1 / 2$ (orientation changes $180^{\circ}$ clockwise along a clockwise path about the point); white asterisks, index $+1 / 2$ (orientation changes counterclockwise for a clockwise path). Two nonvortex points that exhibit poor orientation selectivity and preference for low spatial frequencies are marked with pluses. A closed path enclosing a set of vortices whose indices sum to $N / 2$ traverses $180 \mathrm{~N}$ degrees in orientation: thus, the point labeled a may appear to be a vortex around which orientation cycles by $360^{\circ}$ (index -1 ), but in fact it is not a vortex; there are simply two nearby vortices with index $-1 / 2$ (black asterisks). $B$, Cortical maps of orientation selectivity, orientation gradient (rate of change of preferred orientation), preferred spatial frequency, and ON- or OFF-dominance. Maps of preferred orientation are in Figure $9 B$. The scale of cortical organization is primarily determined by the presence or absence of cortical inhibition ("E" vs "I"). The degree of $\mathrm{ON} / \mathrm{OFF}$ segregation, the presence or absence of cells preferring very low spatial frequencies, and the mean preferred spatial frequency of cells (better visible in $C$ ) are primarily determined by the correlation function (" 0.24 " vs " 0.28 "). Note close correspondence between regions of poor orientation selectivity and regions of high orientation gradient. $C$, Scatterplots of orientation selectivity versus orientation gradient or preferred spatial frequency of individual cells, for the E 0.3 cortices. Scatterplots for 10.3 cortices appear nearly identical for given values of $r_{c}$, and so are omitted. In $B$, orientation selectivity is shown on a gray scale linear from selectivity 0 (black) to 0.16 (white); all higher selectivities appear white. Orientation gradient is linear from 0 (black) to maximum gradient in each picture (white); maxima are 102, 91, 106, and 101 degrees/grid interval from top to bottom. Spatial frequency is shown on a gray scale linear from 0 (black) to the maximum frequency in each picture (white): maxima are $0.156,0.141,0.169$, and 0.159 cycles/grid interval from top to bottom. ON/OFF dominance is the difference between the summed ON-center and summed OFF-center synaptic strengths to a cortical cell; it is shown on a linear scale, where black represents $100 \%$ OFF-center; white, $100 \%$ ON-center; and gray, equality.
} 
A

$E 0.3, r_{C}=0.28$

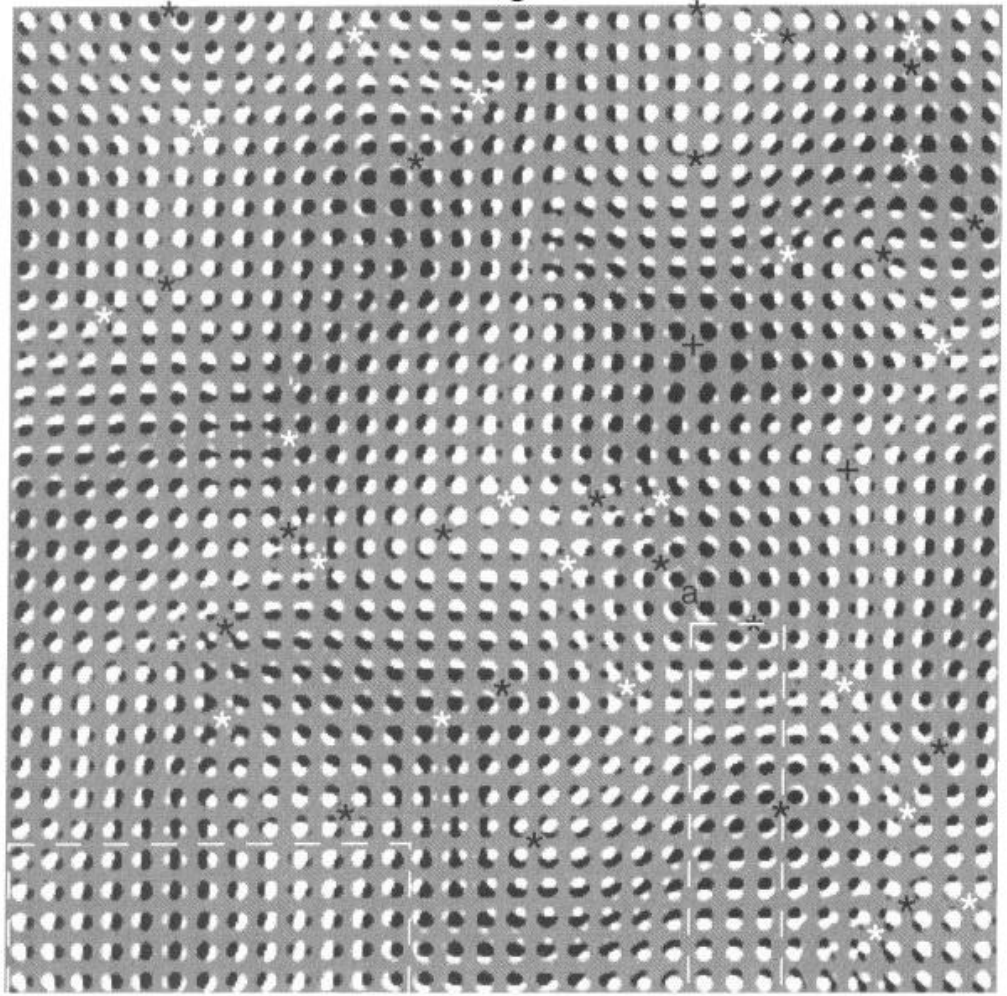

$$
\text { l } 0.3, r_{C}=0.28
$$

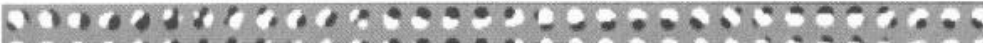

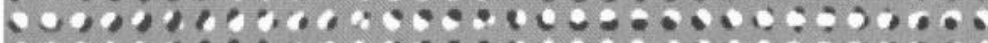

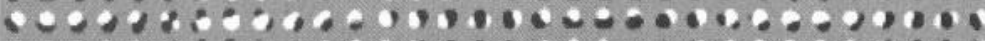

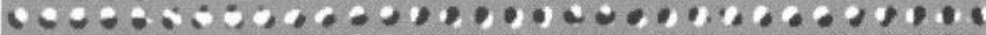

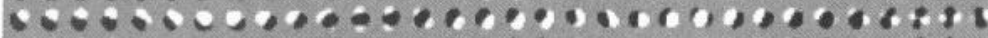

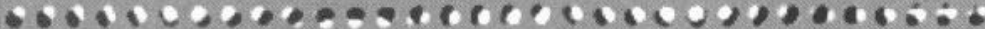

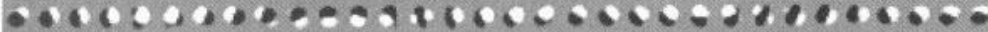

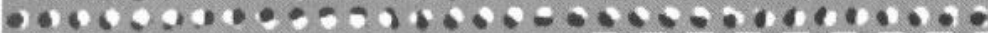

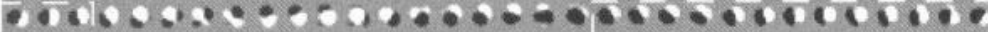

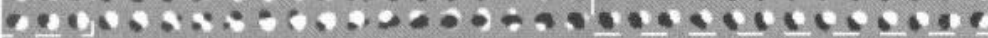

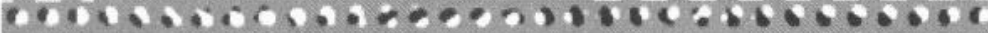

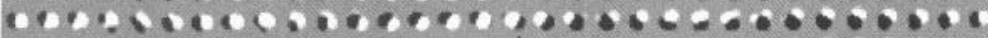

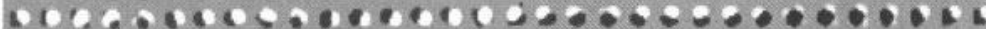

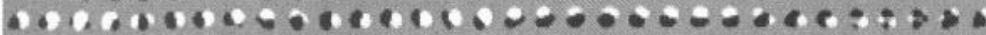

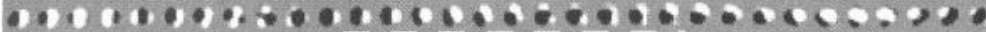

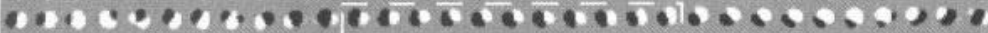
$1000060000+10004000000000000000$

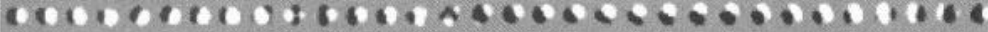

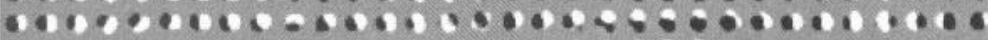

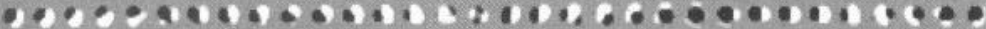

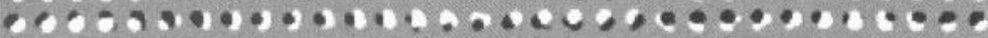

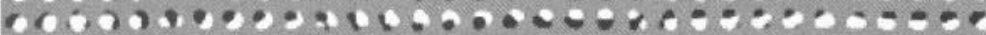

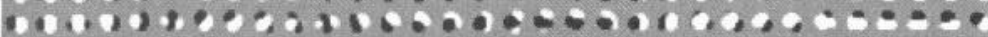

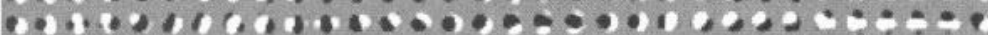

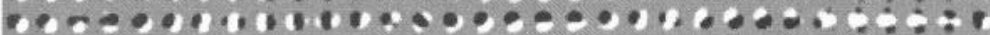

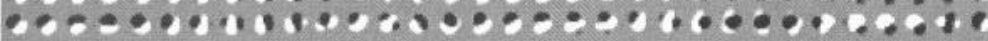

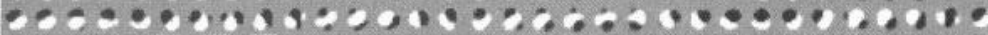

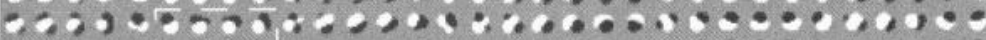

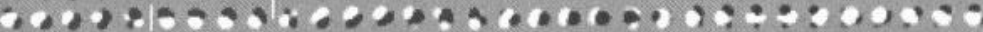

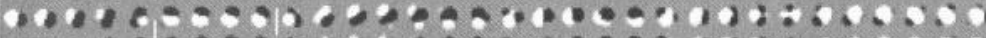

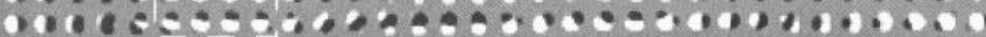

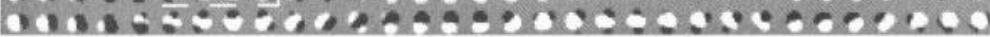

\section{$E 0.3, r_{C}=0.24$}

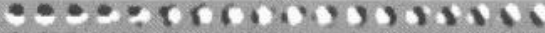
$0.09101000000000 \times$ $4:+01001000000020100$ $6 \div ; 260000,00002000$ atis 00000,0000000

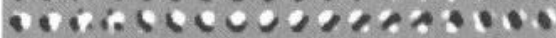

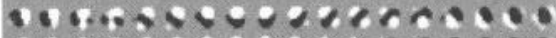

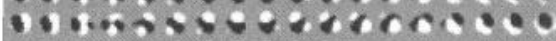

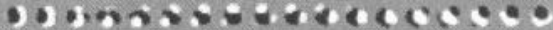

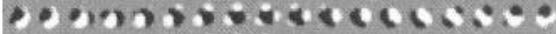

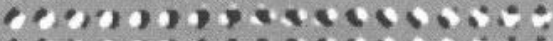
$0,0,001)+200+00,50$

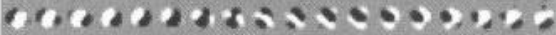

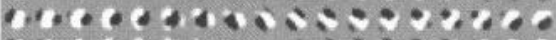

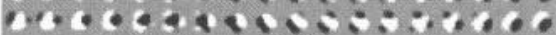

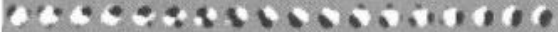

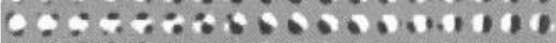

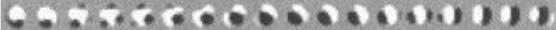

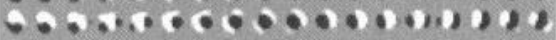

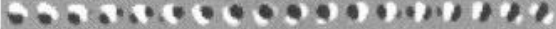

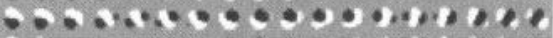

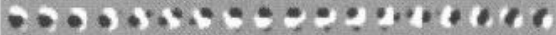

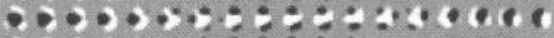

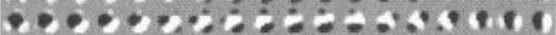

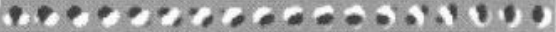

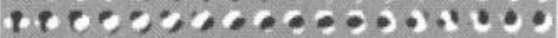

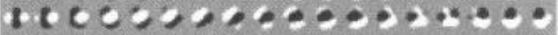

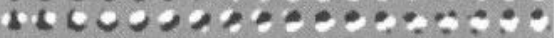

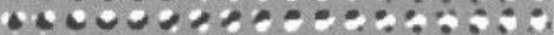

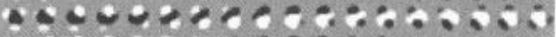

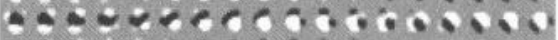

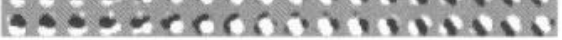

$$
\text { 1 0.3, } r_{C}=0.24
$$

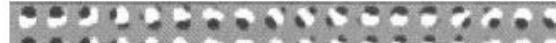

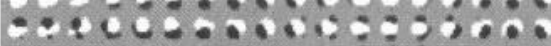

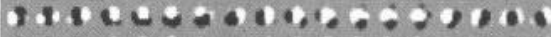

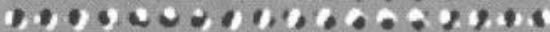

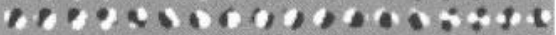
6000.00000000065050

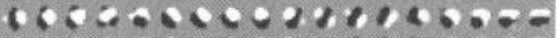
$000+000406000.8=$ $+0,000054000001 \%$

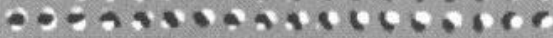
$=2,05000 \times 300600006$ $000,0000000000000 \%$

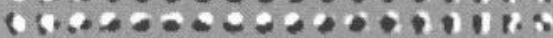

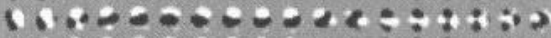

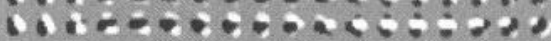

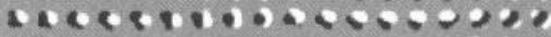
400000000006000000

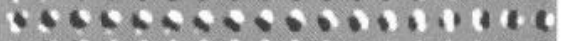

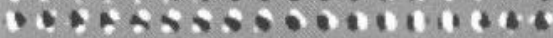

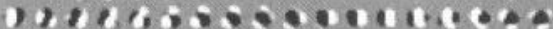

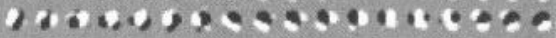

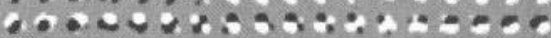

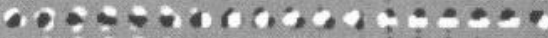

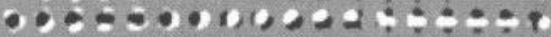

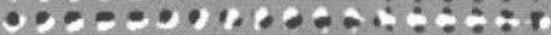

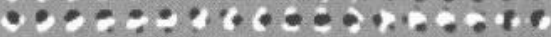

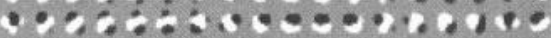

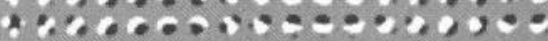

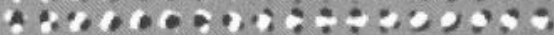

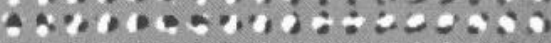

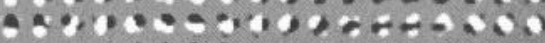
ancos osssececoso 
B

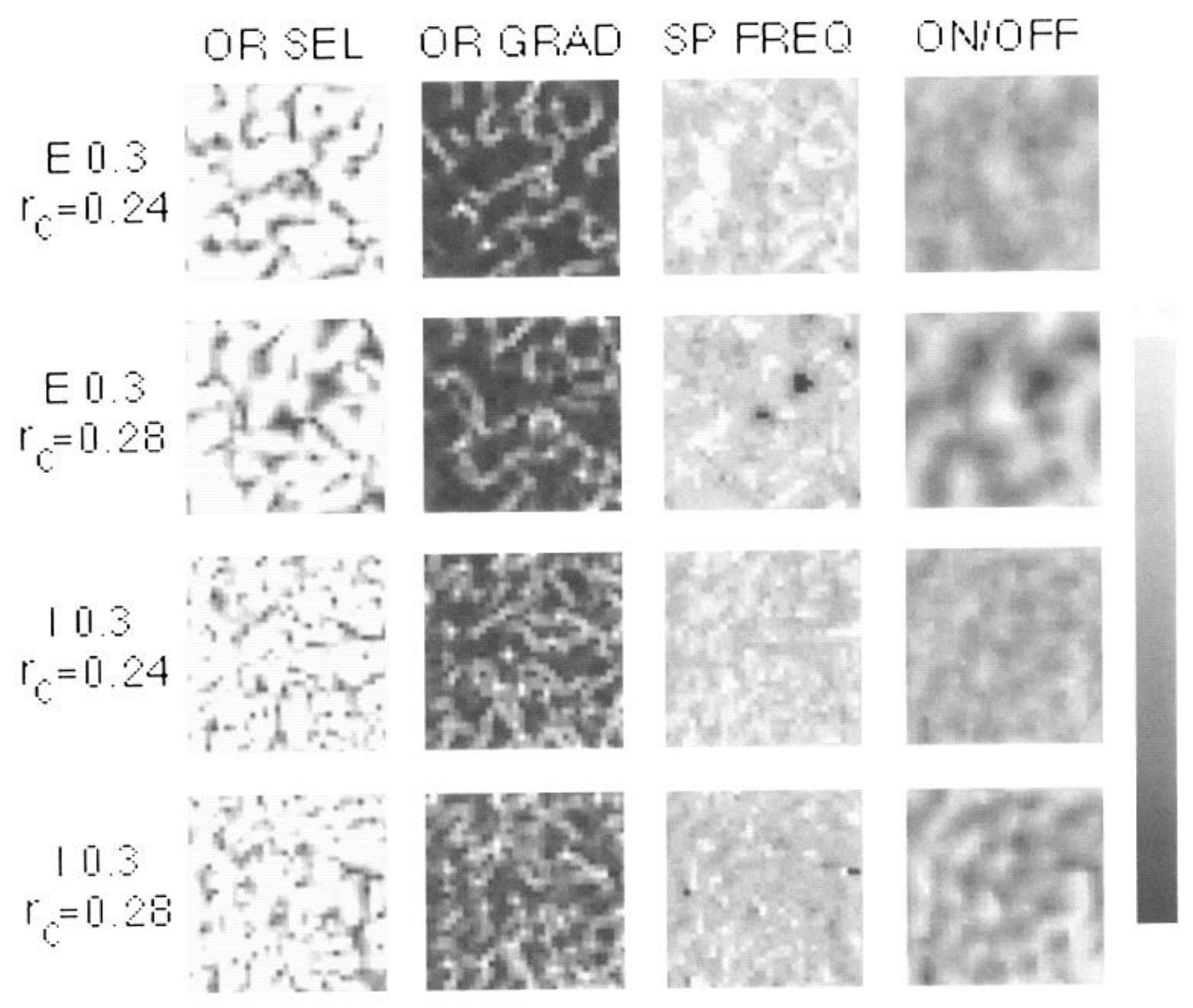

C

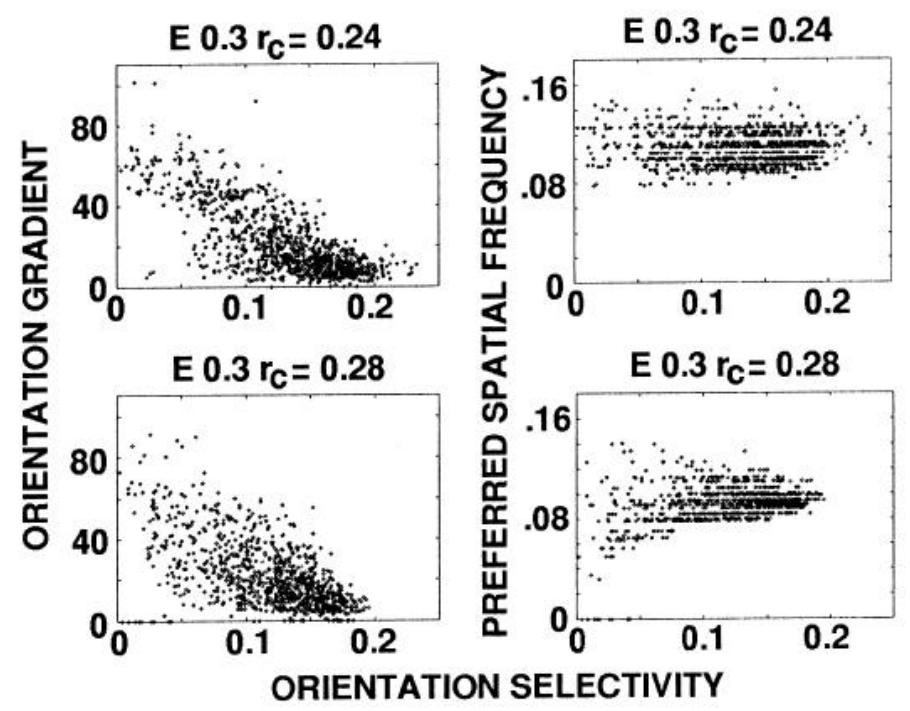




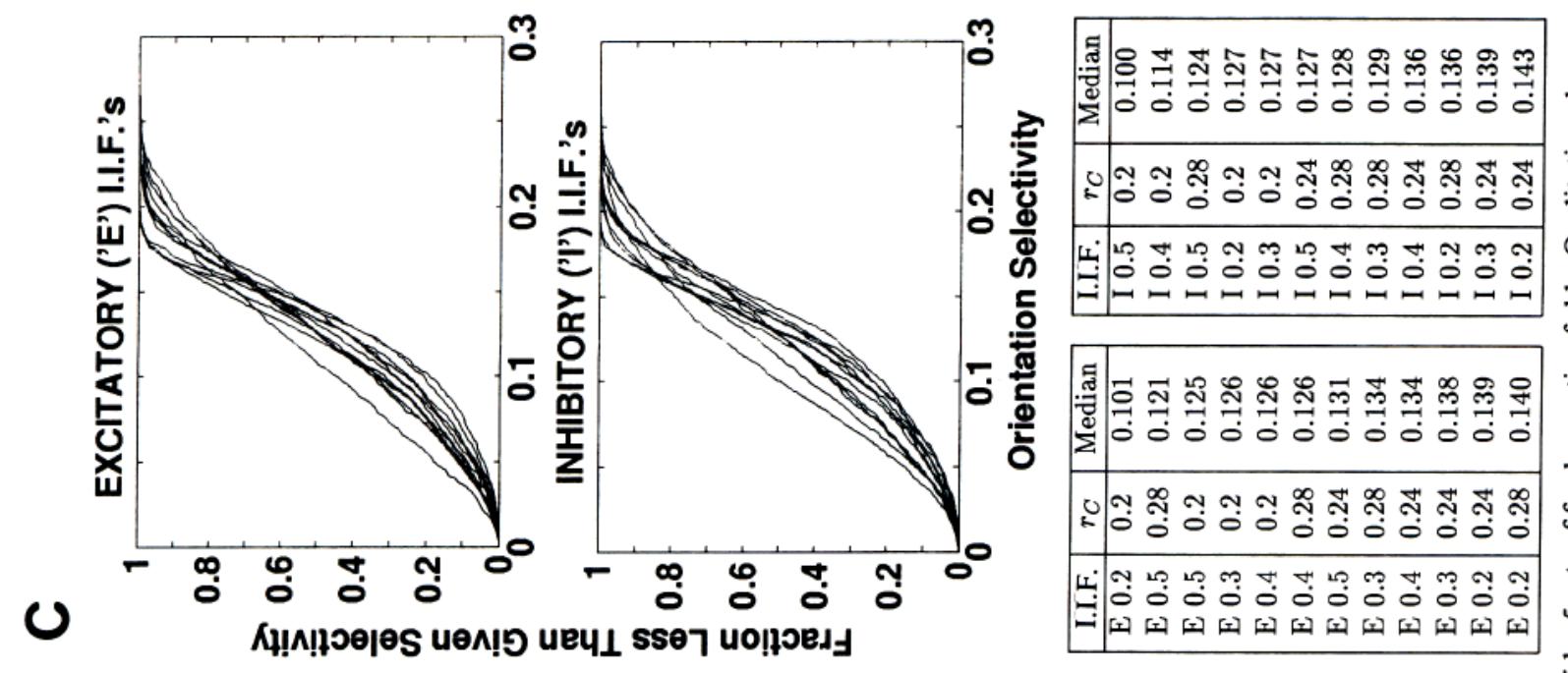

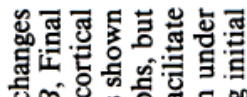

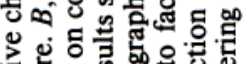

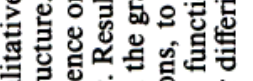

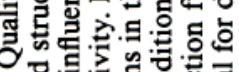

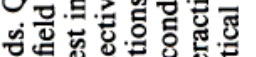

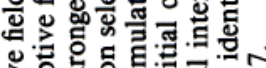

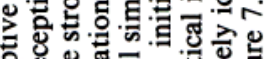

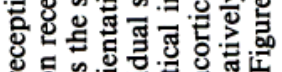

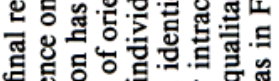

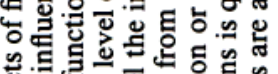

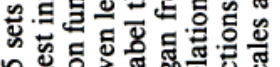

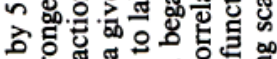

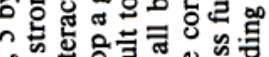

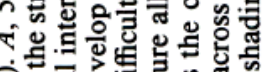

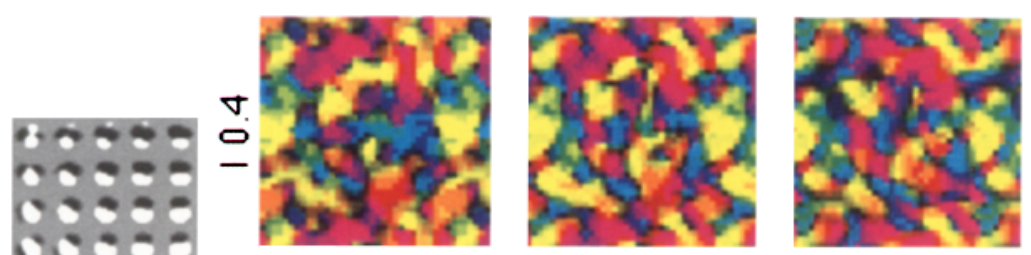

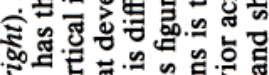

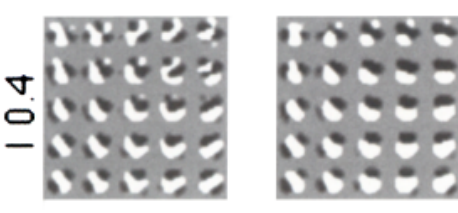

871000 m 82009 0100500

$-100=30$ Nivisea
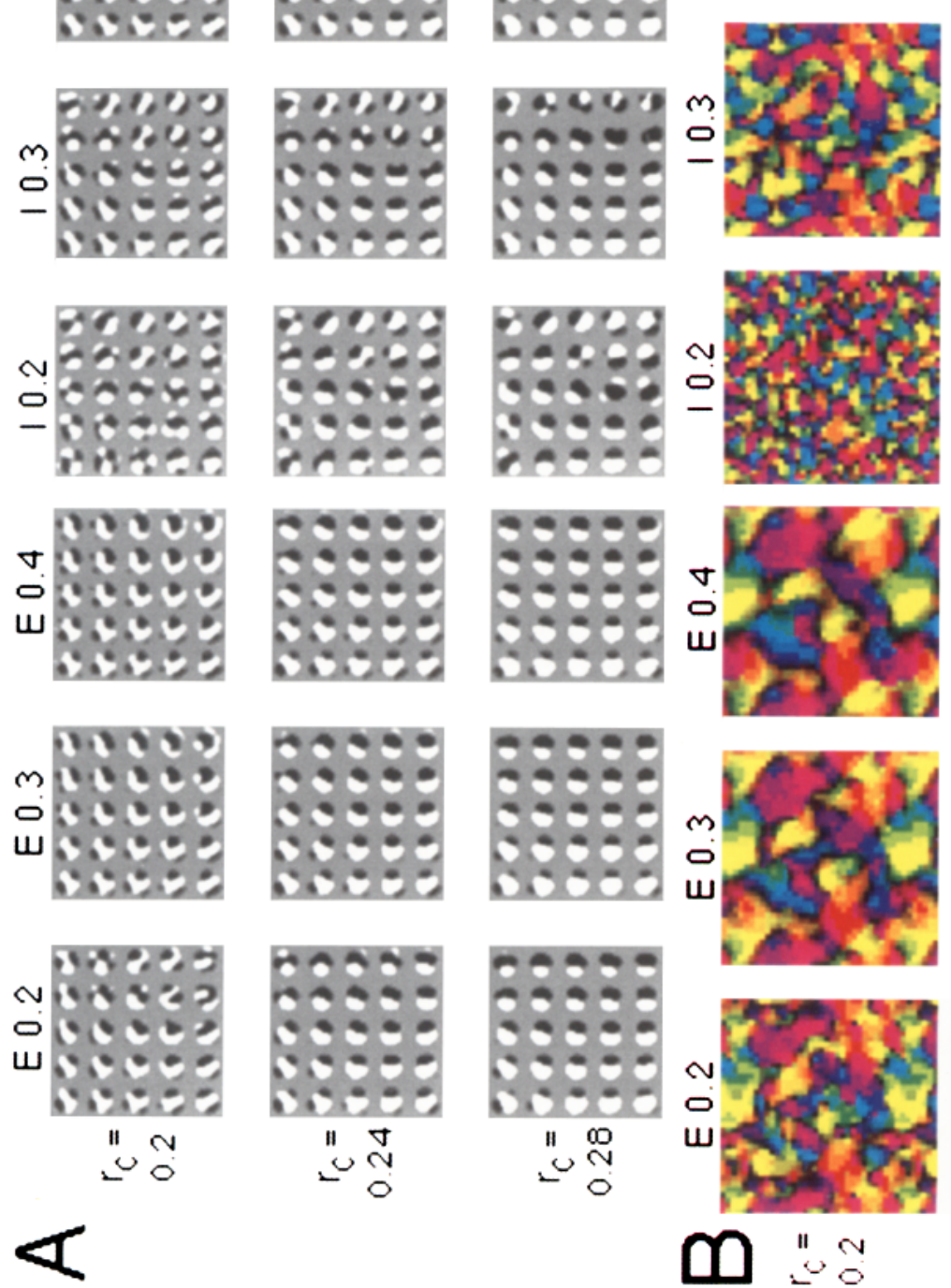
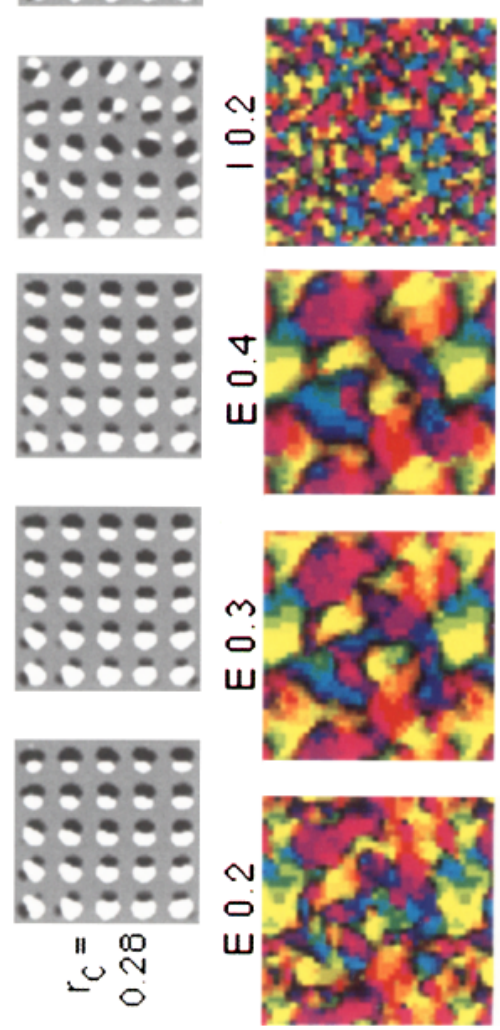

๓
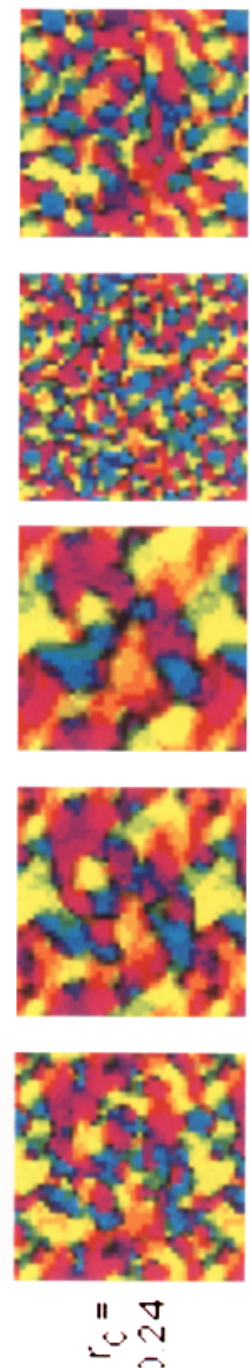
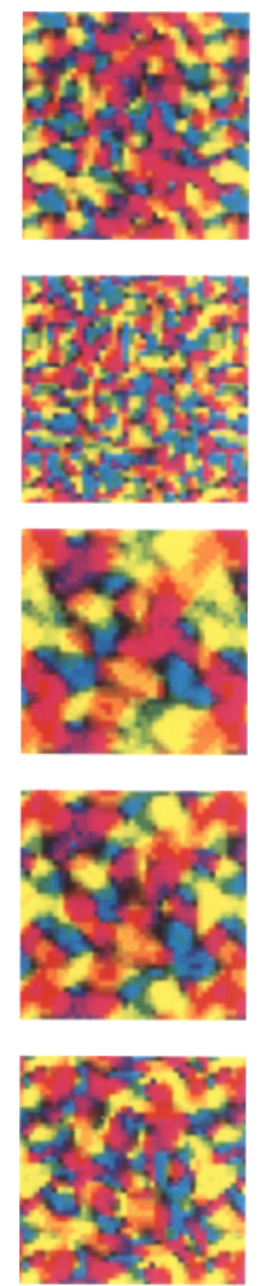

$" \stackrel{0}{0}$

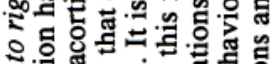

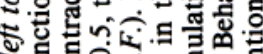

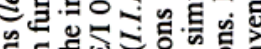

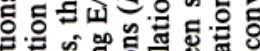
西

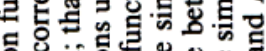

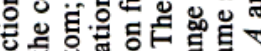
政

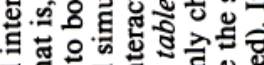

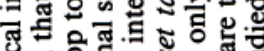

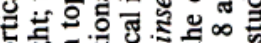

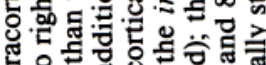

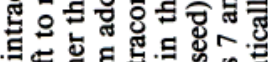

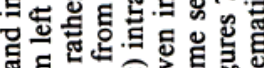
สิ 5.

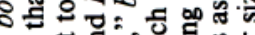

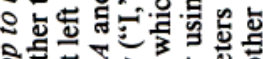
过

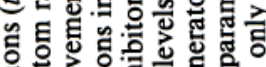

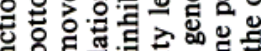

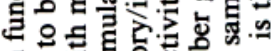
웅 옹

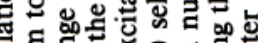

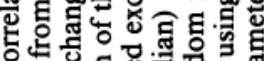

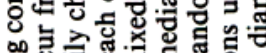
on

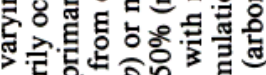

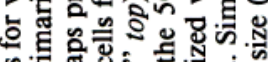

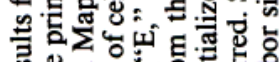

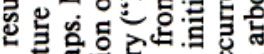

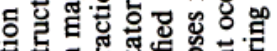
到

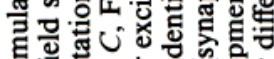

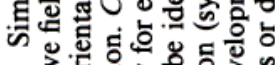

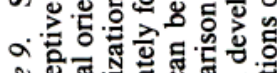

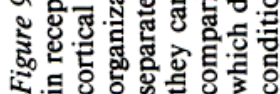



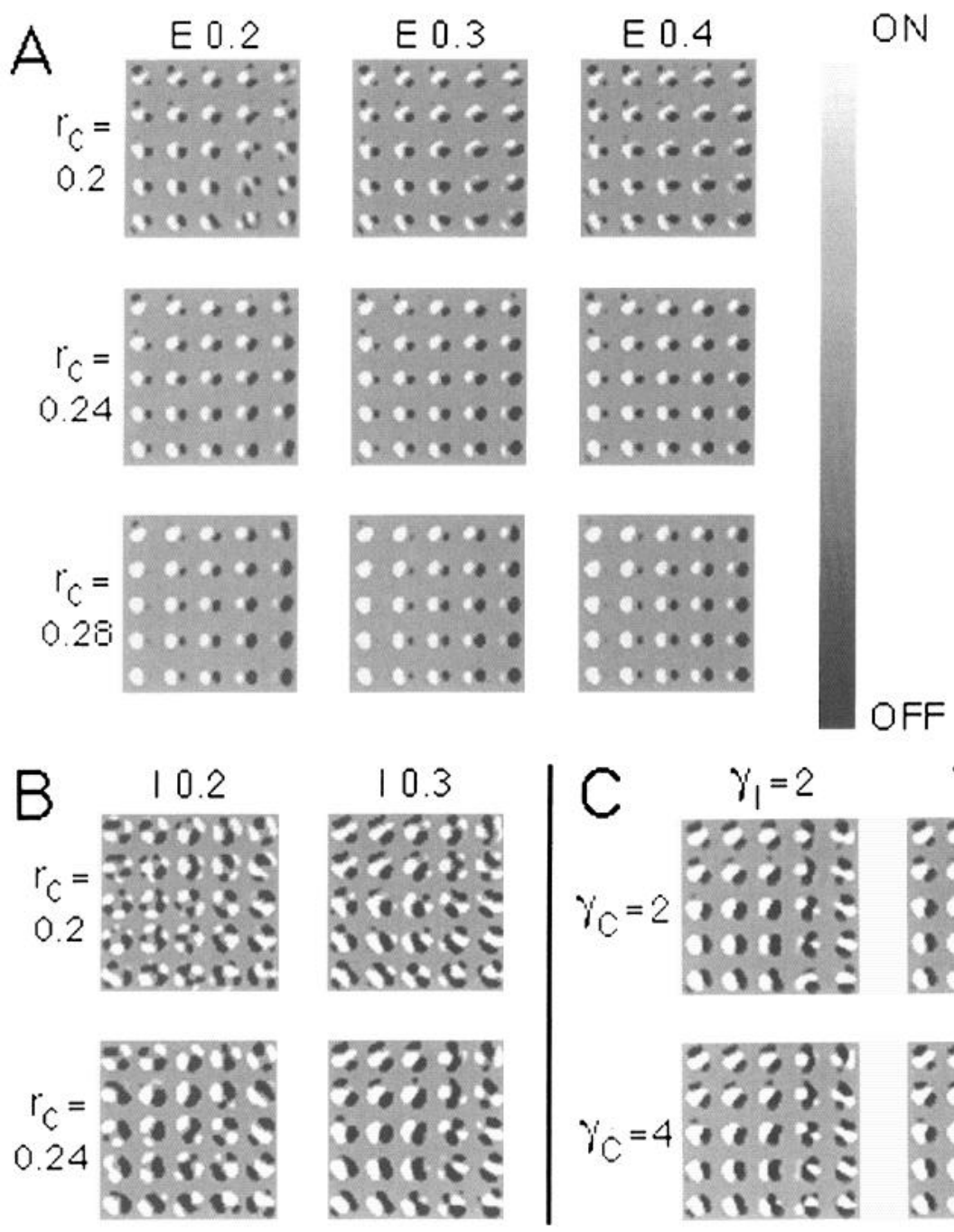

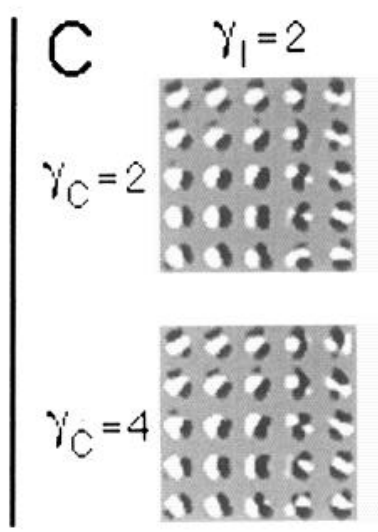

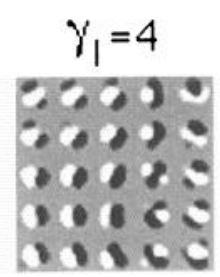

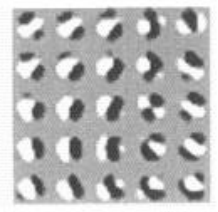

Figure 10. Dependence of simulation results on other parameters. $A$, Effects of varying $s_{\max }$, the maximum synaptic strength. Receptive fields are shown from the same cells as in "E" simulations of Figure $9 \mathrm{~A}$, from simulations identical i all respects except that $s_{\max }$ was equal .o 8 rather than 4 . Effects of change in $s_{\max }$ are qualitatively identical for "I" simulations of Figure $9 A$ (not shown). $B$, Effects of decreasing the arbor parameter $c$, from 0.5 to 0.25 . This decreases the taper of the arbor. In comparison to Figure $9 A$, changes are seen only with the intracortical functions I 0.2 and, to a lesser extent, I 0.3 , and primarily with the narrowest correlation function $r_{c}=0.2$. There are similar but small changes for I $0.2, r_{\mathrm{c}}=0.28$; otherwise, results with either I 0.4 or $r_{c}$. $=0.28$ are essentially unchanged from Figure 9, as are all results with excitatory intracortical functions (not shown). $C$, The parameter $\gamma_{c}$ controls the spread of the inhibitory Gaussian relative to the excitatory in the correlation function; the parameter $\gamma$, controls the same factor in the mixed excitatory/inhibitory ("I") intracortical interaction functions. Each was systematically varied while keeping the peak of the Fourier transform of the corresponding function fixed. Results are essentially invariant, and are shown for $\gamma_{C}, \gamma_{1}=2,4$; compare to $\gamma_{1}=\gamma_{c}=3$ (I $0.3, r_{\mathrm{c}}=0.24$ in Fig. 9A). For $\gamma_{\mathrm{c}}=$ $2,3,4, r$, was set to $0.311,0.24$, and 0.197 , respectively; for $\gamma_{1}=2,3,4, r_{1}$ was set to $0.389,0.3,0.246$. Initial conditions were identical to those in previous figures. Conventions and gray scale are as in Figure $7 \mathrm{~A}$. subregion). However, change of $s_{\max }$ does not alter either the spatial period of ON/OFF alternation within receptive fields or the orientation map.

Variation of other parameters has little effect. The arbor was made less tapered and more like a "pillbox" by setting the parameter $c_{1}=0.25$, rather than 0.5 (see Fig. 5). Receptive fields then receive a greater proportion of their synapses in their periphery. This causes little change in results for most of the choices of $r_{\text {, and }} r_{c}$ used in Figure 9. However, when using the narrowest intracortical interaction functions that include inhibition, this change in $c$, can lead to weakening or elimination of synaptic strength in the centers of receptive fields (Fig. 10B). The parameters $\gamma_{c}$ and $\gamma_{l}$ control the width of the inhibitory relative to the excitory Gaussian in, respectively, the correlation functions and the mixed excitatory/inhibitory intracortical interaction functions. It was expected that the results should depend on only a single length scale for each function, namely, the peak of the function's Fourier transform. To test this hypothesis, $\gamma_{C}$ and $\gamma_{1}$ were varied to take values 2,3 , and 4 , while $r_{c}$ and $r_{t}$ were appropriately covaried to keep the peak of the Fourier transform of each function constant. The results are essentially invariant, as expected, with only a few small changes in outcome (Fig. 10C). The parameter $a_{1}$ sets the strength of intracortical interactions between cells, relative to the strength of interaction among synapses on a single postsynaptic cell. Setting $a_{1}$ to 1 rather than its default value of 0.5 leads to virtually no visible change in results compared to Figure 9 (not shown). Thus, the results primarily depend on the factors that control the length scales of the correlation, intracortical interaction and arbor functions, and the final receptive field diameter, and remain largely invariant as other parameters are varied.

In the model maps that use spatially broad, purely excitatory intracortical interactions, periodic patches of geniculate inputs lose all of their connections to cortex. That is, the geniculate develops spatially periodic alternations between retinotopic regions that project only $\mathrm{ON}$-center input to cortex and regions that project only OFF-center input. Such an outcome can be ruled out biologically, because all retinotopic regions of the LGN project both ON- and OFF-center input to cortex. However, this is likely not to be a serious problem for the model, because simple cells with many different spatial phases are likely to exist in a single vertical column in cortex. If so, the inputs that now disappear would make synapses on the cells with alternative phases. Thus, the problem may be an artifact caused by the inability to include more than one spatial phase at a vertical position in the 2-D cortical model studied here. However, I have also examined the effects on development, within the present framework of a single spatial phase per vertical position, of 


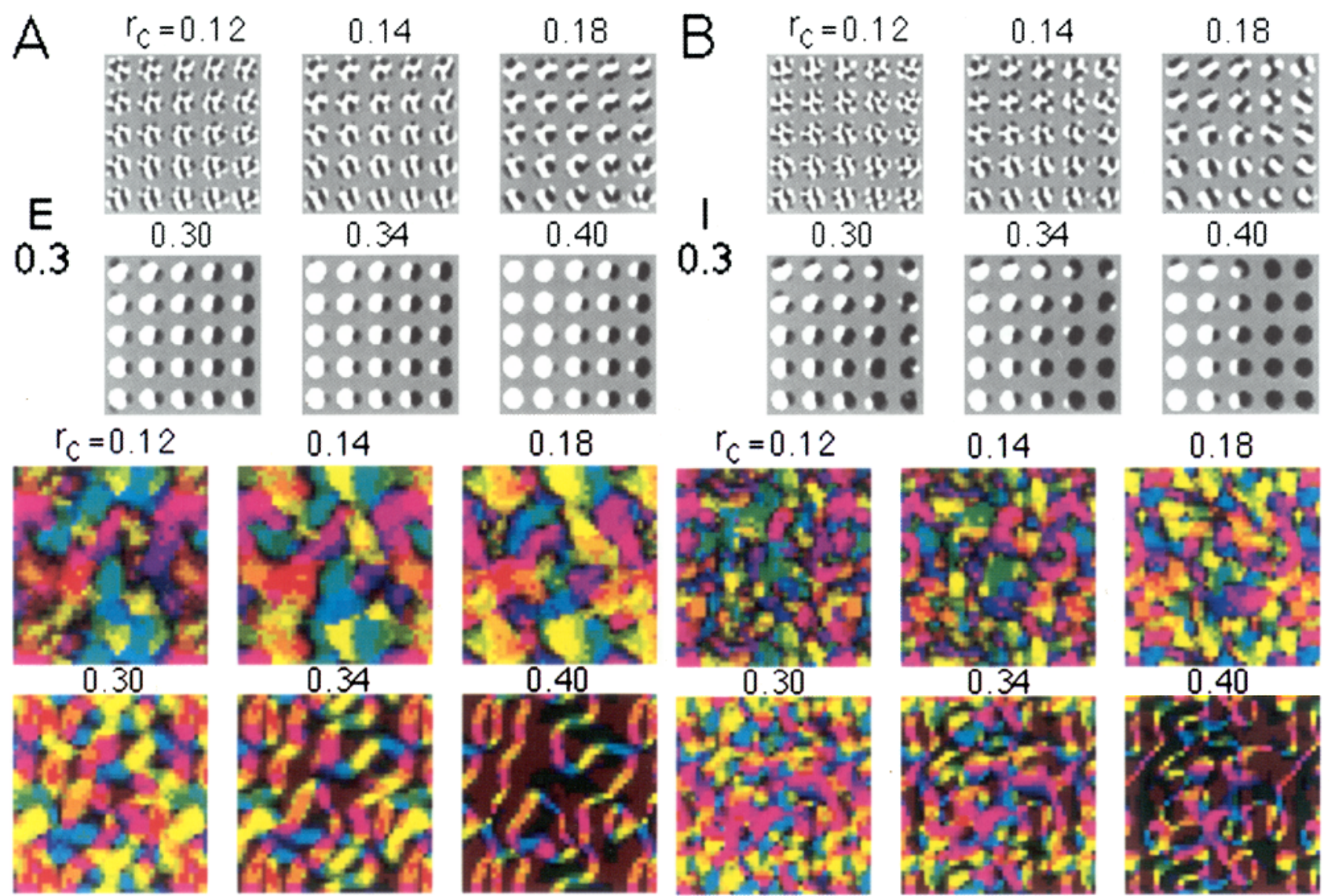

Figure 11. Simulation results for larger variations of the correlation functions: 5 by 5 receptive fields, and orientation maps, from simulations using varying correlation functions. Numbers indicate value of $r_{C}$, which controls the width of the correlation function relative to the arbor (Fig. 5). Results for $0.2 \leq r_{\mathrm{C}} \leq 0.28$ are shown in Figure 9 . $A$, Results using excitatory intracortical interaction function E 0.3 . $B$, Results using mixed excitation/inhibition, I 0.3 . In orientation maps for large $r_{c}$, dark (unoriented) regions represent cells receiving almost exclusively ON- or exclusively OFF-input; at the borders between such regions, oriented cells receiving mixed ON/OFF input can arise. Initial conditions are identical to those in previous figures. Conventions and color scales are as in Figure 7.

a constraint that limits the degree of change in the total synaptic strength over each input arbor to $\pm 50 \%$, so that all inputs retain cortical connections (methods of constraint as described in Miller et al., 1989). Biologically, for example, a less successful input might compete more effectively once it has less total synaptic strength to support, while a more successful input might reach a limit to its ability to compete for further strength. The result (not shown) is very much like that obtained by addition of lateral inhibition to the intracortical interaction function: the spatial scale of orientation domains becomes reduced, but orientation selectivity and its periodic, continuous arrangement develop as in the simulations presented here.

\section{Determinants of orientation selectivity and preferred spatial frequency}

The results of more widespread variation of the width of the correlation function, using either the purely excitatory intracortical interaction function E 0.3 or the mixed function I 0.3 , are shown in Figure 11. A range of values of $r_{c}$ leads to strong development of orientation selectivity (see also Fig. 12B). Whenever orientation selectivity develops, there is periodic and continuous organization of preferred orientation across the cortex. With decreasing breadth of correlations (decreasing $r_{c}$ ), orientation selectivity decreases, because the width of $\mathrm{ON}$ - and
OFF-subregions narrows. This yields multiple ON- and OFFsubregions within a receptive field, which often fail to maintain straight lines or a common direction. This decrease of orientation selectivity with decreasing $r_{C}$ is nearly identical using larger arbors (diameter 25; not shown), even though the width of individual ON/OFF subregions is then larger for a fixed $r_{c}$. Thus, this decrease of orientation selectivity is due to the number of ON/OFF subregions in a receptive field, and not to the width of the subregions relative to the grid used for simulations. With increasing breadth of correlations, orientation selectivity decreases because all-ON or all-OFF receptive fields develop. That is, a transition is made to the parameter regime in which inputs segregate between postsynaptic cells rather than within receptive fields. Oriented cells can nonetheless develop at the boundaries (snake-like colored regions in cortices with largest $r_{C}$ ) of cortical regions of all-ON or all-OFF receptive fields (dark regions) when the correlation function is not too broad. This regime might be appropriate to describe the ferret, where ONand OFF-center inputs are segregated across cortex (Zahs and Stryker, 1988) and only $40 \%$ of cells in the mature layer 4 are orientation tuned (Chapman, 1991).

Across parameters, where orientation selectivity strongly develops, the mean preferred spatial frequency of cortical cells in simulations is accurately determined by the peak of the Fourier 


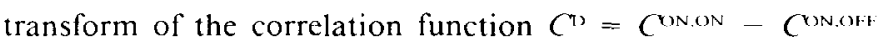
(Fig. 12.1), as theory predicts (Fig. 2). Orientation selectivity develops most strongly for values of $r_{c}$ (Fig. 12B) that yield about two to three ON/OFF subregions at the mean preferred spatial frequency. Because of the taper of the arbor, the effective arbor diameter (the cutoff of the Fourier transform of the arbor function) is approximately 11.3 grid intervals; two subregions

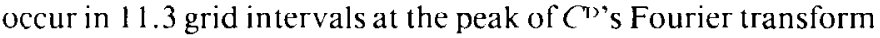
for $r_{c}=0.29$, while three subregions occur for $r_{c}=0.19$.

The prediction that the mean preferred spatial frequency of cortical simple cells is given by the peak of the Fourier transform of $\left({ }^{\top}\right)$ can be compared to existing experimental measurements by making several assumptions, as follows. The function $C^{(7)}$ describes dark correlations measured as a function of input separation and center types in, for example, the LGN of early postnatal kittens. Such measurements have not been made, but similar measurements have been made in the retinas of adult cats (Mastronarde, 1983a,b). There, like-center-type correlations were found to be positive, and opposite-type correlations negative, both falling to zero at a certain separation $L(\theta) / 2$ where $\theta$ is receptive field eccentricity. Dark correlations were not measured at further separations, so it is unknown whether a "Mexican hat" structure exists in the adult retina; if not, such a structure might nonetheless be induced in the LGN through inhibitory LGN circuitry (see Discussion).

There is no guarantee that correlations in the young animal resemble those in the adult (see Discussion). However, possessing only the experimentally measured length scales $L(\theta)$, let us hypothesize that these also characterize the young animal, and determine whether the resulting prediction is consistent with visual cortical receptive fields. Thus, assume that $\left({ }^{\top}\right)$ is "Mexican hat" in the LGN of young animals, that its central positive region falls to zero over the same distance $L(\theta) / 2$ as in adult retina, and that it is negative over some comparable further distance. Then the simplest estimate of the peak of the Fourier transform of ( ${ }^{1)}$ is that it occurs at frequency 1 cycle $/ 2 L(\theta)$ (Fig. 13.1). To see why, note that the peak of (1)'s Fourier transform corresponds to the frequency of the cosine that best fits (") (i.e., $\cos k \cdot x$ that maximizes $\left.\int d^{2} x C^{1}(x) \cos k \cdot x\right)$. This cosine will not differ greatly from the cosine that has the same zero-crossings as $\left({ }^{D}\right.$, which has frequency 1 cycle/ $2 L(\theta)$. Across a wide variety of Mexican hat functions constructed as a difference of Gaussians, this estimate is within $20 \%$ of the true peak (Fig. 13B).

To apply this estimate to visual cortex, I assume that $\mathrm{X}$-cell correlations determine simple cells in cat area 17, and that $Y$-cell correlations determine those in area 18 (Ferster, 1990a,b; Ferster and Jagadeesh, 1991). Further details are in Methods. I then compare the estimate 1 cycle $/ 2 L(\theta)$, derived from Mastronarde's measurements for $\mathrm{X}$ - or $\mathrm{Y}$-cells, respectively, to measurements made by Movshon et al. (1978b) of the preferred spatial frequencies of cat area 17 or area 18 cortical cells (Fig. 13C). The matches across eccentricities are reasonable. The error bars show the standard deviation in the predicted mean resulting only from the fact that the measured correlations vary with polar angle at a fixed eccentricity (Mastronarde, 1983a, and personal communication). Several additional factors could account for the variability in the biological data. First, the biological data include both simple and complex cells from all laminae; variability is presumably less for simple cells in layer 4 , the subject of the present model (the standard deviation for simple cells from all laminae in area 17 was $56 \%$ of the mean; Movshon et al., 1978b). Second, the intracortical interactions induce variability, as shown
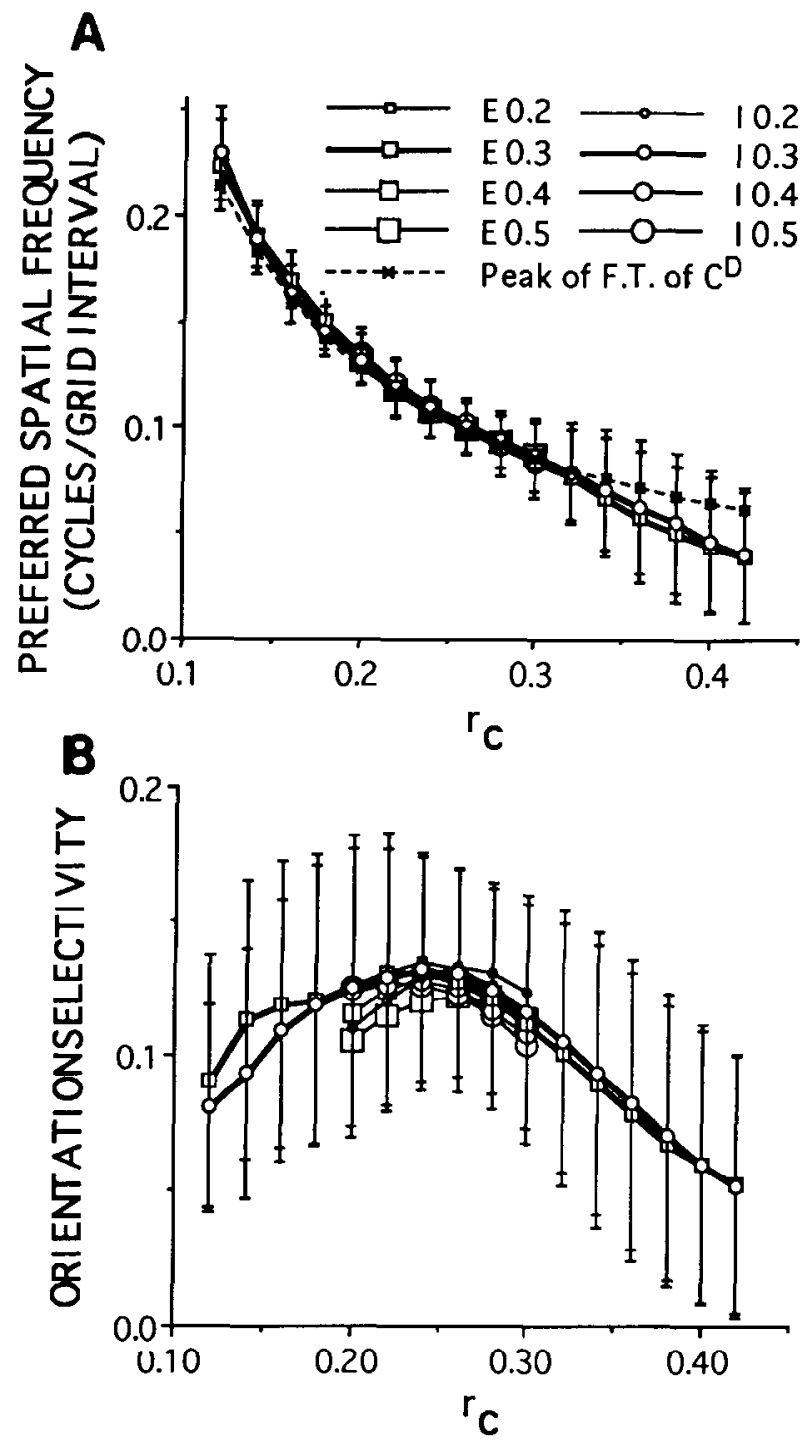

Figure 12. Dependence of best spatial frequency and orientation tuning on parameters. $A$, Best spatial frequency of cortical cells in simulations, versus $r$. (parameter controlling width of correlation functions). Dashed line with $x$ 's shows the theoretical prediction. given by the spatial frequency of the peak of the Fourier transform (F.T.) of the correlation function ( ${ }^{(1)}$; the match is excellent except at large $r_{c}$, where orientation selectivity is poor and many all-ON or all-OFF cells (preferred spatial frequency 0 ) develop. $B$, Orientation selectivity of cortical cells in simulations, versus $r_{c}$. In both $A$ and $B$, each symbol represents the mean (of best frequency or orientation selectivity, respectively) over all cells from a single simulation; error bars are standard deviation, and are shown only for E 0.3 and I 0.3 (error bars for other simulations for a given $r_{c}$ are similar). Heary solid lines connect results using intracortical interaction functions $\mathrm{E} 0.3$ or $\mathbf{I} 0.3$; light lines connect results for other intracortical interaction functions, which were simulated only for $r_{c}=$ $0.2-0.3$. These simulations all began from the same initial condition, and include those displayed in Figures 7-11. Results using other initial conditions or other arbor sizes (only arbor diameter 11 has been systematically studied) are essentially identical.

in Figure $8 B$ (the standard deviation of best spatial frequency is $9-18 \%$ of the mean for the simulations shown in Fig. 9; it is higher for lower or higher values of $r_{c}$ ). Third, there will be biological variability in ( ${ }^{\mathrm{T}}$ at a fixed eccentricity and polar angle.

Thus, the prediction made by the model for the mean preferred spatial frequency of cortical simple cells is quantitatively consistent with existing experimental measurements. A rigorous 
Figure 13. Prediction of the mean preferred spatial frequency of cortical simple cells. $A$, Outline of the prediction method. The model predicts that the mean preferred spatial frequency of cortical simple cells is that which maximizes the Fourier transform $(F . T$.$) of$ $C^{\mathrm{D}}$. This is the frequency of the cosine (dotted line) that best fits $C^{\text {T) }}$ (solid line) Existing data (Mastronarde 1983a,b) only give an estimate of $L$, the diameter of the positive central region of $C^{3}$. The best-fitting cosine should have similar zero-crossings to $C^{7}$, and therefore should have frequency about $1 /(2 L)$. $B$, Accuracy of $1 /(2 L)$ as an estimate of the peak of the Fourier transform of a "Mexican hat" function. Data are shown for 2-D difference of Gaussian functions of the form $I(x)=\exp \left(-x^{2}\right)-\left(I_{h} /\right.$ $\left.I_{n}{ }^{2}\right) \exp \left(-x^{2} / I_{n}{ }^{2}\right)$. The parameters $I_{n}$ and $I_{\text {w }}$ are labeled I Height and I Width, respectively, in the figure. $I_{1 \text {, }}$ determines the lateral spread of inhibition relative to excitation; $I_{h}$ determines the strength of inhibition, with $I_{h}=1$ corresponding to exact balance between inhibition and excitation. Across a wide range of parameters, provided there is sufficient inhibition to transform $I(x)$ from a Gaussian $\left(I_{h}=0\right)$ to a difference of Gaussians, $1 /(2 L)$ is within $20 \%$ of the true peak. $C$, Comparison of model prediction to experiment. Experimental data are from Figure 6 of Movshon et al. (1978b), reproduced by permission. The data include both simple and complex cells from all laminae. On these data are superimposed the curves predicted by the model for the mean preferred spatial frequency of layer 4 simple cells. Possible sources of variance in spatial frequency, most of which are not included in the error bars shown, are discussed in Results. Vertical position of $Y$-cell curve has further uncertainty due to variability in Mastronarde's results; see Methods.
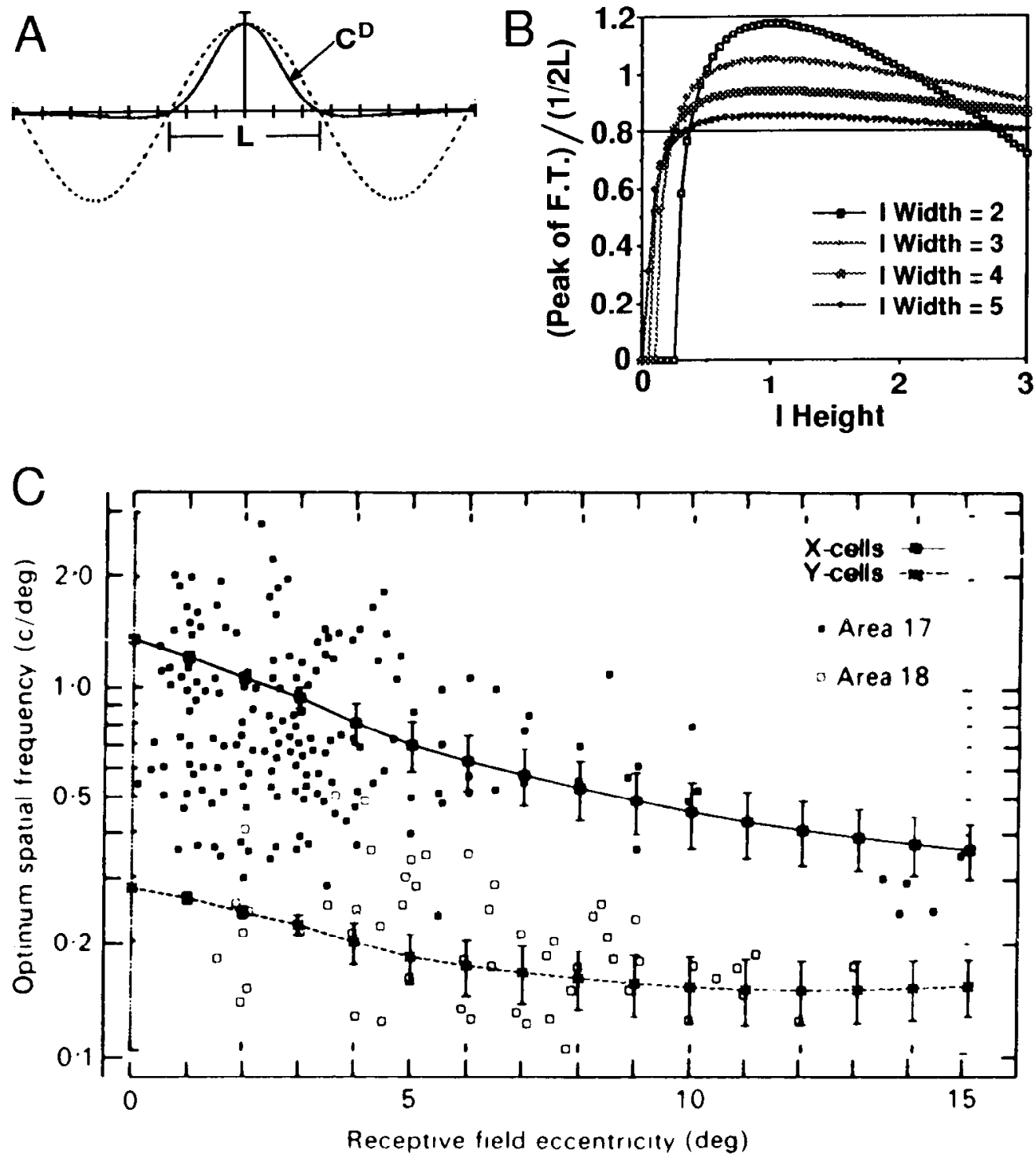

test of this prediction will require direct measurements of $C^{T}$ in the LGN of young animals.

\section{Determinants of the orientation map}

While the properties of individual receptive fields are largely determined by the correlation functions, their arrangement into a map of preferred orientation is most influenced by the intracortical interaction function. The quantitative dependence of the spatial period of preferred orientation on the parameters can be studied by Fourier transforming the maps of preferred orientation to determine the amount of power at each spatial period or frequency. Here, spatial period refers to the distance in grid intervals across the model cortical map corresponding to one complete cycle of orientations. Frequency is the inverse of spatial period, that is, cycles of preferred orientation per grid interval. In Figure 14, the frequency containing peak power and the width at half-height of the frequency versus power curve are displayed across parameters. The model maps tend to include a broader range of frequencies, and more low frequencies, than experimental maps. It is possible that this is an artifact of the necessarily small size of the model maps rather than a feature intrinsic to development under the model.

Broader spread of excitatory intracortical influences, or of the central excitatory region of intracortical interactions that include lateral inhibition, results in larger iso-orientation domains and correspondingly smaller frequencies. In contrast, there is little dependence on the correlation function. The peak frequency does not correspond directly to the peak of the Fourier transform of the intracortical interaction function, but instead is somewhat higher. For example, the peak of the $\mathbf{I} 0.3$ function is at 0.09 0.1 cycles/grid interval, while simulations with this function tend to have maximum power at a frequency about $50 \%$ higher; and the peak of the excitatory functions is at frequency zero, while the corresponding simulations have maximum power at a nonzero frequency. There appears to be a maximum spatial period (minimum frequency) for arbitrarily long-range excitatory interactions. The maps of preferred orientation for arbor diameter 13 change only slightly as $r_{1}$ is increased beyond 0.5 , although orientation tuning decreases (not shown). Theoretical arguments can be made as to why, once excitatory interactions 

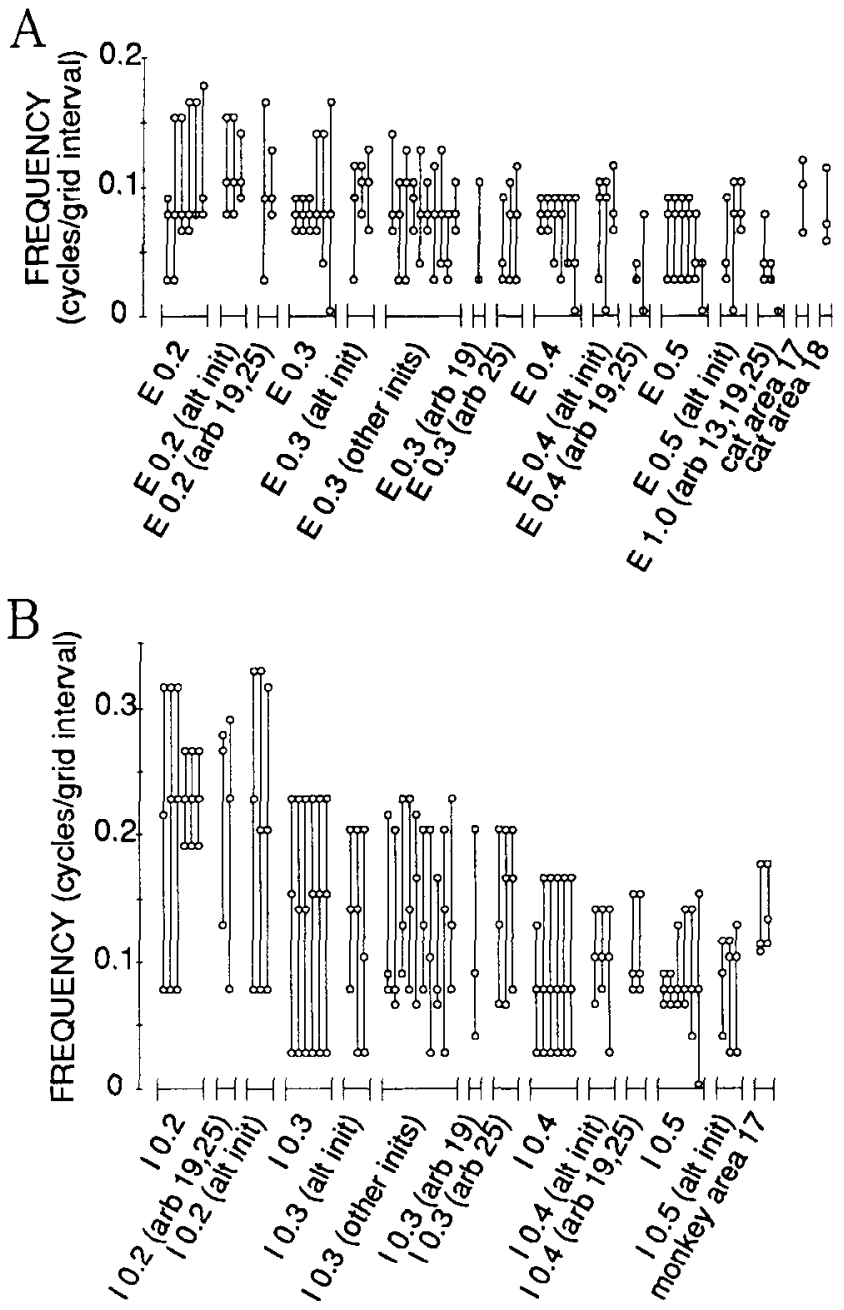

Figure 14. The periodicity of the simulated orientation maps. Each vertical line shows the results of the Fourier transform of one simulated map. The middle circle on each vertical line shows the frequency containing maximum power; the other two circles show the highest and lowest frequencies containing at least $50 \%$ of this maximum. Frequency is the number of cycles of orientation per grid interval of the model cortical map; a frequency of 0.1 cycles/grid interval corresponds to an orientation map that traverses a full cycle $\left(180^{\circ}\right)$ of orientation every 10 grid intervals. $A$, Results with excitatory ("E") intracortical interactions. $B$, Results with mixed excitatory/inhibitory ("I") intracortical interactions. The frequency primarily depends on the intracortical interaction function. Relative lack of dependence on the correlation function is seen in bins simply labeled "E $r_{i}$ " or "I $r_{r}$ ": these each show results for six correlation functions, from left to right, $r_{c}=0.2,0.22$, $0.24,0.26,0.28,0.3$. These simulations all began from the same initial condition, and include those displayed in Figures 7-9. Relative lack of dependence on initial conditions is shown by bins labeled (alt init). These were all done with a single, alternate initial condition; each bin shows, left to right, results for $r_{c}=0.2,0.24,0.28$. Bins labeled (other inits) show results of 10 additional random initial conditions, all for $r$, $=0.24$. Relative lack of dependence on the arbor function, except with wider excitatory intracortical interactions, can be seen in bins labeled arb 19 or arb 25. These show results using arbor diameters 19 and 25, which are much like the results for arbor diameter 13 for most intracortical interaction functions. For E 0.3 and I 0.3 , the arbor 25 bins show, left to right, $r_{c}=0.2,0.24,0.28$; arbor 19 bins used $r_{c}=0.24$. For other intracortical interactions, only $r_{\mathrm{c}}=0.24$ was studied with these arbor sizes, and arbors 19 and 25 are shown left to right (for $\mathrm{E}$ 1.0 , arbors 13,19 , and 25 are shown left to right, all with $r_{c}=0.24$ ). Results from four experimental maps of orientation are shown at the right; $92 \mu \mathrm{m}$ in the experimental maps is equated with 1 grid interval extend over about an arbor radius, maps of preferred orientation should not change as excitatory interactions are made increasingly long range. This maximum spatial period increases as the arbor and correlation functions are made larger in spatial extent (the correlation function scales with the arbor function for fixed $r_{r}$ ), which increases the distance over which cells can directly influence one another's development. The period otherwise shows little dependence on the arbor diameter for a fixed intracortical interaction function.

The model cortical maps qualitatively resemble experimental cortical maps (Fig. 15A). Maps of preferred orientation, with regions of high orientation gradient marked as white, are shown for model cortices and for experimental maps from monkey area 17 (unpublished data from Ts'o et al., 1990) and cat area 18 (from Bonhoeffer and Grinvald, 1991). The model and experimental maps are shown on an identical scale if 4 pixels, or $92 \mu \mathrm{m}$, in the experimental map correspond to one grid interval in the model. This is a reasonable but arbitrary choice, corresponding to arbors with a diameter of about a millimeter and intracortical excitation over a radius of about 200-300 $\mu \mathrm{m}$. To compare the maps on this scale, the model maps have been filled in by linear interpolation on a grid four times finer than the model grid. This represents a hypothesis, which can be tested as computational resources increase, that simulation on a finer grid would result in such smooth filling in.

At least three points of resemblance exist between model and experimental cortices. First, the spatial scales on which orientation periodically varies are similar in the model and corresponding experimental maps. This simply reflects the choice of parameters in the model maps displayed. Second, both model and experimental maps have a modular structure and include many "pinwheels" or vortices of orientation. This is difficult to quantify, but is likely simply to reflect a lack of significant structure of the orientation map beyond the basic periodicity, that is, a mixing in Fourier space of periodic patterns of orientation with similar frequency but many directions and phases. This can be seen in Figure 15B, which shows the power at each 2-D Fourier frequency of the maps in Figure $15 \mathrm{~A}$. Model and experimental maps have quite different distributions of power, but have in common similar peak and cutoff frequencies and mixing of many directions in Fourier space, and these apparently are sufficient to yield visual resemblance of the maps. Third, in the monkey and corresponding model map, regions of high orientation gradient ("fractures") tend to run in lines (Blasdel and Salama, 1986), while in the cat and corresponding model map, fractures are largely restricted to points or short lines in the regions of orientation vortices. The theoretical determinants of this difference are not clear, although purely excitatory intracortical connections seem necessary in the model to achieve

in the model maps, as described in Results and the Figure 15 caption. Given this scale, the cat cortices have periodicity similar to the E 0.3 cortices, while the monkey cortices have periodicity similar to I 0.25 (not shown) or I 0.3 cortices; however, the model maps tend to have a broader range of frequencies and a greater representation of very low frequencies than the experimental. Experimental maps were provided as follows: cat area 17, by Dr. T. Bonhoeffer (unpublished observations); cat area 18 , by Drs. T. Bonhoeffer and A. Grinvald (from Bonhoeffer and Grinvald, 1991); monkey area 17, left, by Dr. D. Ts'o (unpublished data from Ts'o et al., 1990); monkey area 17, right, by Drs. E. Bartfeld and A. Grinvald (unpublished observations). Cat 18 and left monkey maps are shown in Figure 15. 


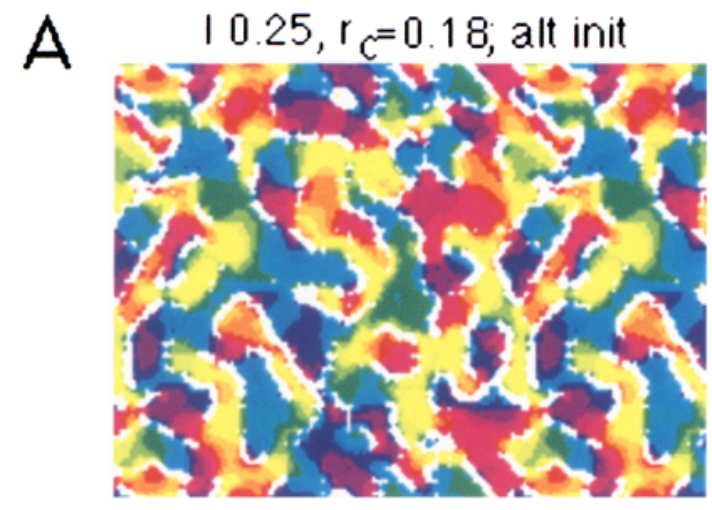

$\mathrm{E} 0.3, \mathrm{r}_{\mathrm{C}}=0.24$; other init

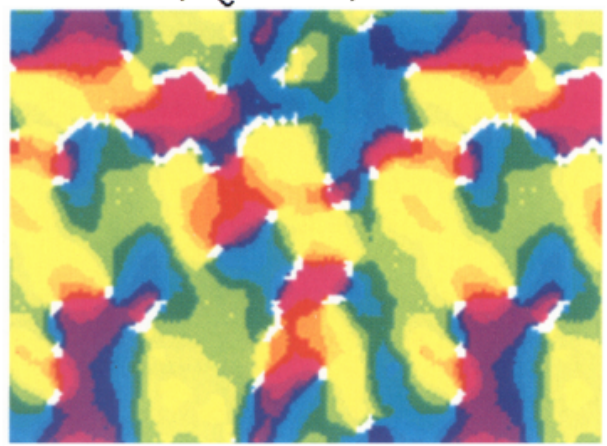

Monkey $\mathrm{v} 1$

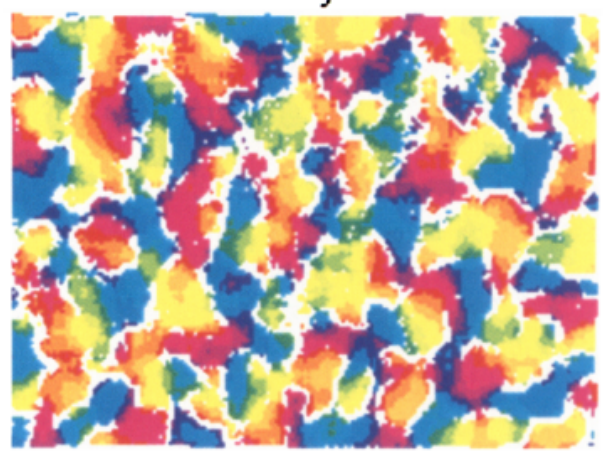

Cat Area 18

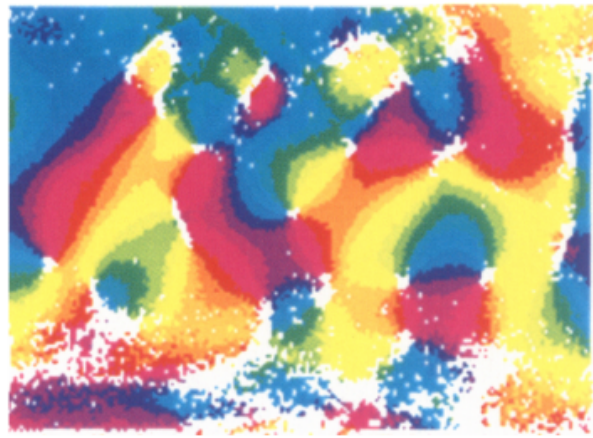

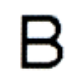
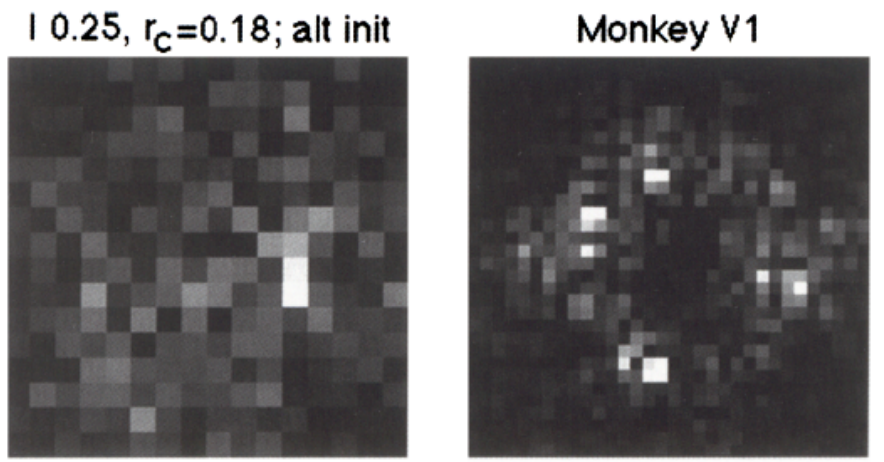

$E 0.3, r_{C}=0.24$; other init

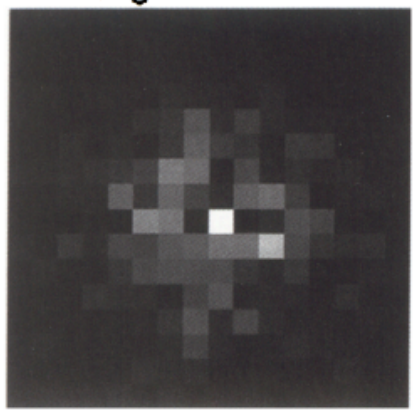

Cat Area 18

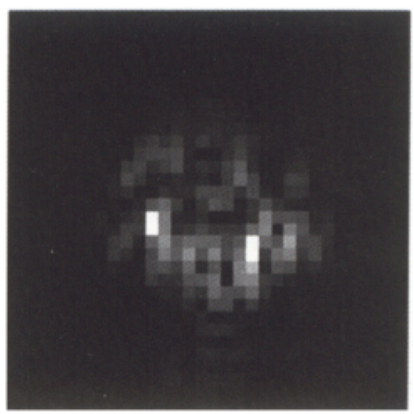

Figure 15. Comparison of model and experimental orientation maps. A, Comparison of model maps to maps of monkey V1 and cat area 18. Hue signifies preferred orientation (color scale is as in Fig. $7 \mathrm{~B}$, but without brightness variation). Points of high orientation gradient are shown as white. One grid interval in model maps corresponds to $92 \mu \mathrm{m}$ in experimental maps. Model maps show 47 by 35 grid intervals (note wraparound); experimental maps are 4.32 by $3.22 \mathrm{~mm}$. Points colored white have gradient $\geqq 64^{\circ}$ per model grid interval or per $92 \mu \mathrm{m}$. Model maps have been filled in by linear interpolation (see Results), and gradients were computed after interpolation. $B, 2$-D power spectra for each of the maps in $A$. White indicates high power; black, low power. Spectra are shown on identical spatial scale (i.e., points a given distance from the center of each spectrum represent the same wavelength) if one model grid interval equals $92 \mu \mathrm{m}$. However, "pixel size" in model spectra is twice as large as in experimental. Power at a point $(x, y)$ in model spectra, or $(2 x, 2 y)$ in experimental (measured in pixels from the "center"-below) corresponds to a periodic pattern of orientation with a complete cycle of orientation in $\left(32 / \sqrt{x^{2}+y^{2}}\right)$ grid intervals or in $\left(2.94 / \sqrt{x^{2}}+y^{2}\right)$ mm. Pixel sizes differ because Fourier transforms were 128 by 128 for model maps and 256 by 256 for experimental (see Methods). Illustrated are central $N$ by $N$ pixels of full spectra, where $N=16$ (model) or $N=32$ (experimental). This contains essentially all of the power. "Center" is $(0,0)$ in coordinates in which 
point rather than line fractures. It is also not yet clear whether this difference in fracture patterns is a general feature of experimental cortical maps. Thus, lacking quantitative characterization of the features common to experimental maps and of the conditions necessary theoretically to achieve such features, strong conclusions cannot be drawn from the resemblance of model and experimental maps.

\section{Analysis of the model}

Mathematical analysis of the model will be presented elsewhere (K. D. Miller, unpublished observations). Here I summarize a few central results.

Under a correlation-based mechanism, pairs of cortical cells with excitatory intracortical interactions tend to develop correlated receptive fields, while those with inhibitory intracortical interactions tend to develop anticorrelated receptive fields (Miller, 1990a). The degree of correlation or anticorrelation of two simple cells depends not simply on their relative orientations, but on the relative retinotopic positions of each cell's ON- and OFF-subregions (Fig. 16). Therefore, the organization of preferred orientation is determined by a coupling between the orientation and the spatial phase of receptive fields.

Heuristically, the patterns of synaptic connectivity that develop are determined by both the correlation function, $C^{\circ}$, and the intracortical interaction function, $I$, as follows (Miller, 1990a; Miller and MacKay, 1994). $C^{\mathrm{D}}$ approximately determines the spatial period of alternation between ON-and OFF-center inputs across receptive fields, as has been shown. Similarly, $I$ determines the spatial period of alternation between $\mathrm{ON}$ - and OFF-center inputs across arbors, that is, across the projection to cortex from a single retinotopic position in the LGN. Each of these two alternations occurs with spatial period determined by the peak of the Fourier transform of the corresponding function. The spatial phase of receptive fields within an iso-orientation cluster tends to shift in a regular, periodic manner. This oscillation of spatial phase is determined by the sum of the receptive field and arbor oscillations (locally, the wavenumber of the spatial phase oscillation is the vector sum of the wavenumbers of the receptive field and arbor oscillations).

When $I$ is purely excitatory, there is an oscillation across receptive fields but not across arbors. Then receptive fields in an iso-orientation cluster will change spatial phase steadily with movement across cortex perpendicular to the preferred orientation, as seen in Figure $8 . A\left(\mathrm{E} 0.3, r_{c}=0.28\right)$. This change in spatial phase occurs with a spatial period, across the cortex, that is equal to the spatial period of alternation of ON- and OFFinputs within individual receptive fields. This maintains in retinotopic register the ON-subregions and the OFF-subregions of nearby cells, and keeps all receptive fields as correlated with one another as possible. This optimally matches the receptive fields to the purely excitatory intracortical interactions. On the other hand, when (") and $I$ are both Mexican hat and are similar
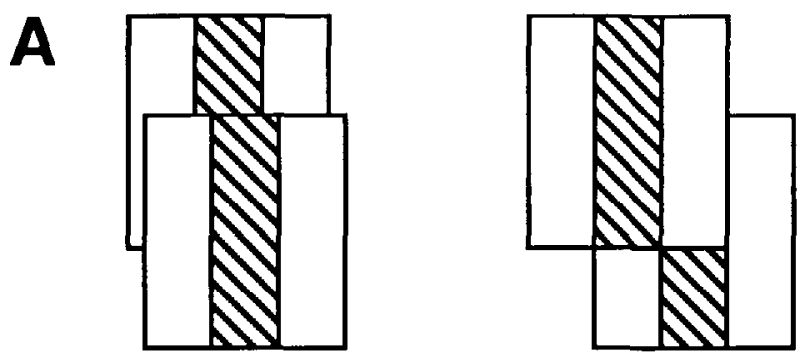

B
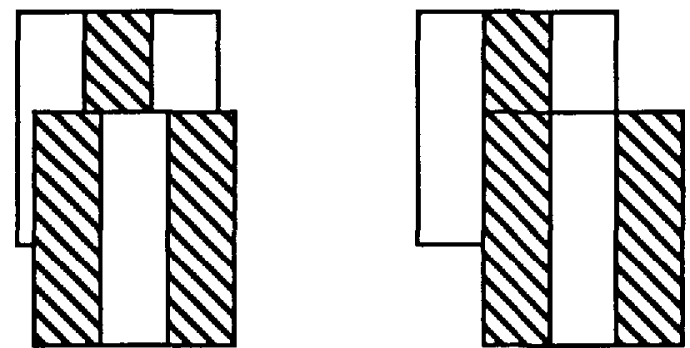

Figure 16. Correlation of simple cell receptive fields at varying retinotopic separations. $A$, Two receptive fields with identical preferred orientations and spatial phases (stripes signify ON-regions; white, OFFregions). Left, At small retinotopic separations, $\mathrm{ON}$-regions overlap and OFF-regions overlap; hence, the responses of the two receptive fields to dark input activity are well correlated. Right, At a larger retinotopic separation perpendicular to the orientation axis, the ON-regions of one overlap the OFF-regions of the other, so the responses of the two receptive fields are maximally anticorrelated. $B$, Two receptive fields with identical preferred orientations but opposite spatial phases. The situation is reversed from $A$ : at small retinotopic separations the responses of the two receptive fields are anticorrelated, while at larger retinotopic separations perpendicular to the orientation axis they are correlated. Thus, interactions between simple cells crucially depend on spatial phase and cannot be described in terms of their preferred orientations alone.

in spatial extent, the receptive field and arbor oscillations may cancel, yielding little or no shift in spatial phase of receptive fields within an iso-orientation cluster. This provides an cxplanation for the lack of phase shift sometimes seen in simulations in such cases (Fig. 8A, I $0.3, r_{c}=0.28$ ). Intuitively, the reason is as follows. The cortical distances corresponding to the minimum of $I$ and the minimum of $C$ are similar in this case. $\Lambda t$ this distance, intracortical interactions are maximally inhibitory, and two identical receptive fields are maximally anticorrelated. Hence, in this case a pattern in which spatial phase does not vary within an iso-orientation cluster can give an optimal match between the correlations of receptive fields and the intracortical interactions (although other, more complicated patterns may also be optimal).

It is possible to characterize the fastest-growing patterns of synaptic connectivity in the model [the eigenfunctions with largest eigenvalue of the operator determining development of $S^{\mathrm{I}}(x$, $\left.\alpha) \equiv S^{\mathrm{ON}}(x, \alpha)-S^{\mathrm{OFF}}(x, \alpha)\right]$. These fastest-growing patterns dominate the early, linear phase of the development of $\mathrm{ON}$ -

lower left corner is $(-N / 2,-N / 2)$, upper right is $(N / 2-1, N / 2-1)$. Each spectrum is shown on a linear gray scale from zero power (black) to maximum power in that cortex (white). Model cortices are named as in Figure 14. Resemblance of model and experimental maps appears to be robust for these intracortical interaction and correlation functions, across varying initial conditions and (without rescaling the correlation function) for arbor size 11 as well as 13. There is one exception: excitatory intracortical interactions may produce "fractures" (regions of high orientation gradient) that are somewhat more linear than in the illustrated model cortex, depending on the initial condition. Map of monkey area 17 provided by Dr. D. Ts'o (unpublished data from Ts'o et al., 1990); map of cat area 18 provided by Drs. T. Bonhoeffer and A. Grinvald (from Bonhoeffer and Grinvald, 1991). 
and OFF-domains, and features common to all of the fastestgrowing patterns and their mixtures are likely to persist in the final pattern despite biological nonlinearities (see discussion in Miller, 1990a,c, 1992a; Miller and Stryker, 1990).

The features just discussed - the spatial periods of the alternations of $\mathrm{ON}$ - and OFF-center strength in receptive fields and in geniculate projections, and of the oscillation across cortex of receptive field spatial phase-are typically common to all of the fastest-growing connectivity patterns. Thus, the predictions of these features in terms of $C^{D}$ and $I$ are likely to hold independent of nonlinearities. The determinants of the final periodic map of preferred orientation are only partly solved, and a quantitative characterization of its spatial period is lacking. However, this period is not common to the fastest-growing patterns. Rather, it appears to emerge as a predictable, statistical result of random mixtures of those patterns. Nonlinearities may select nonrandom mixtures. For this reason, the spatial period and other features of the orientation map might be significantly altered under more realistic nonlinearities.

Finally, why does the oscillation of ON- and OFF-center subregions within receptive fields lead to orientation selectivity, rather than to a center-surround (see Fig. 2) or other structure? There appear to be two reasons. First, for a cortical cell developing in isolation, there are typically several optimal (fastestgrowing) receptive field patterns, which are nearly equally well matched to the correlations and which grow at nearly equal rates. Most combinations of these yield orientation-selective cells; that is, a random initial mixture of these patterns will favor an orientation-selective outcome. Second, the presence of intracortical interactions between cells favors an orientationsclective outcome: receptive ficlds consisting of oriented subregions can more readily be matched between many neighbors than can fields consisting of concentric circular subregions. Thus, the optimal patterns for the cortex as a whole consist largely or exclusively of oriented cells.

\section{Discussion}

The present results demonstrate that a simple, activity-dependent competition between ON- and OFF-center inputs is sufficient to account for the development of orientation- and spatial frequency-selective simple cells and their arrangement into periodic, continuous cortical maps. The primary requirements are the existence of a Mexican hat correlation function between $\mathrm{ON}$ and OFF-center inputs, and interaction between the competitions occurring on nearby cortical cells. Given these conditions, the results arc robust, occurring over a wide range of model parameters and independent of choice of random initial condition. The structure of receptive fields is primarily determined by the correlation function $C^{\mathrm{D}}=C^{\mathrm{ON} . \mathrm{ON}}-C^{\text {ON.OFF. The mean }}$ preferred spatial frequency of simple cells is determined by the peak of $C$ 's Fourier transform. This is consistent with experimental measurements of preferred spatial frequencies of visual cortical cells and of correlations among adult retinal ganglion cells. When this preferred spatial frequency is $1-1.5$ cycles (two to three ON/OFF subregions) per arbor diameter, excellent orientation selectivity results. The cortical maps of orientation selectivity, spatial frequency, and other properties are primarily determined by interactions within cortex, but the input correlations and arborizations also contribute. The spatial phases of simple cells can play a key role in the organization of cortical maps. Possible origins for other features of cortical organization, such as blobs, pinwheels, and afferent ON/OFF segregation, have been suggested.

The model does not produce the final precision of visual cortical receptive fields or of cortical organization. Some receptive fields in the model, particularly those along boundaries between orientation domains, have curved or odd, nonoriented structures, unlike those reported for simple cells. The model orientation maps appear to be less regular than cortical maps, containing a wider range of frequencies including. in particular, more long wavelengths. Thus, the model is far from complete. Many additional mechanisms, including but not limited to nonlinearities in activation and plasticity rules, plasticity of intracortical connections, vertical intracortical interactions, and the particular biophysical properties and connectivities of specific cell types, will need to be considered in attempting to account for such precise structure.

\section{Predictions of the model}

Two levels of prediction arise from the present work. Strong predictions derive directly from the hypothesis that simple cell receptive fields arise from an ON/OFF competition in the presence of a Mexican hat correlation function: if these predictions are wrong, the model is wrong. These include the predictions that Mexican hat correlations should be found experimentally, that development of simple cells should depend on the presence of such correlations, and that the mean preferred spatial frequency of simple cells should correspond to the peak of the Fourier transform of $C^{D}$. If the additional assumption is made that the couplings between simple cells are direct, and not mediated via nonsimple cells (discussed below), then the additional strong prediction is obtaincd that the arrangement of preferred orientations across cortex should be coupled to the arrangement of receptive field spatial phase. Other results are much weaker, as they depend on additional assumptions such as the 2-D, distance-dependent model of cortical interactions or the simple saturating nonlinearities used here; these results can be discarded without discarding the basic hypothesis. These include the determinants and structure of the cortical maps of orientation and other receptive field properties, and the determinants of the degree of orientation selectivity. I will discuss each of these predictions in turn.

\section{Activity dependence and correlation structure underlying simple cell development}

The hypothesis that activity-dependent competition between $\mathrm{ON}$ - and OFF-center inputs accounts for the development of cortical orientation selectivity can be experimentally tested in several ways; negative results would clearly falsify the hypothesis. First, orientation-selective cells should not form if all neural activity in the retinas, LGN, or cortex is blocked sufficiently early in development. Second, a Mexican hat correlation structure between ON- and OFF-center inputs should be found in dark activity in the preorientation layer at the appropriate developmental time. Third, manipulations that abolish a Mexican hat correlation structure between $\mathrm{ON}$ - and OFF-center inputs without abolishing neural activity should, if initiated before the development of orientation selectivity, prevent such development. An example is artificial stimulation of the optic nerves while retinal neural activity is blocked, which has been accomplished but at a later developmental age (Stryker and Strickland, 1984). These tests will be difficult to perform due to the early developmental age at which orientation selectivity develops. 
However, indirect evidence for a Mexican hat correlation structure may be sought through studies of LGN dark activity at later developmental times.

In the model, convergence of ON- and OFF-inputs onto the same cortical cells results in simple cell receptive fields. Therefore, the model predicts that the ON- and OFF-center cells of the monkey parvocellular cortical layer $4 \mathrm{C} \beta$ should arise in the regime of segregation onto different postsynaptic cells; that is, the ON-center $4 C \beta$ cells should receive only ON-center LGN excitatory input, and similarly for OFF-center cells. Failure of this prediction may not be fatal to the hypothesis, because in principle center-surround receptive fields can also develop in the regime in which $\mathrm{ON}$ and OFF converge (Fig. 2), but such failure would present a very strong challenge to the hypothesis, because such an outcome is not expected without specific nonlinear effects that select such patterns over orientation-selective patterns.

\section{Preferred spatial frequencies of cortical cells}

A strong prediction of the hypothesis is that the mean preferred spatial frequency of cortical simple cells should correspond to the peak of the Fourier transform of $C^{1}$. Based on Figure 13, this implies that in cats, the developmental $C^{D}$ in LGN should have length scales similar to those derived from adult retinal correlations.

\section{Principles underlying the orientation map, and coupling to spatial phase}

The cortical organization of orientation that results under the present hypothesis may be altered as more complexity is added to the model of intracortical circuitry. However, two firm conclusions may be drawn. First, the proposed mechanism is sufficient to achieve continuity, periodicity, and other basic spatial structure in the orientation map. Second, if orientation selectivity develops by the hypothesized correlation-based mechanism, the cortical organization of receptive fields is shaped by the tendency of cells with excitatory intracortical interactions to develop correlated receptive fields and of cells with inhibitory intracortical interactions to develop anticorrelated receptive fields. Two simple cells are predicted by the model to be correlated if they have spatially overlapping ON-subregions and overlapping OFF-subregions (Fig. 16). They are anticorrelated if their receptive fields "anti-overlap," that is, if the ON-subregions of one overlap the OFF-subregions of the other.

Biologically, simple cells receive "opponent inhibition"; that is. ON-subregions receive $O N$-excitatory input and OFF-inhibitory input, and conversely for OFF-subregions (Palmer and Davis, 1981a; Mullikan ct al., 1984a,b; Ferster, 1988). Inhibition can only come from other cortical cells (Ferster and Lindstrom, 1983), and such phase-specific inhibition could only come from other simple cells. Thus, it appears that when one simple cell inhibits another, the two develop "anti-overlapping" receptive fields, as the theory predicts. Similarly, the very fact that simple cells maintain cleanly segregated excitatory subregions despite intracortical excitation suggests that when one simple cell excites another, the two develop overlapping ONsubrcgions and overlapping OFF-subrcgions.

If the map of preferred orientation develops through horizontal coupling between simple cells, then the model predicts that this map emerges from a deeper principle than local continuity of orientation. Rather, the map emerges from a tendency to maximize ON-overlap and OFF-overlap between cells that excite one another, and to maximize "anti-overlap" between cells that inhibit one another. Thus, studies of the simultaneous arrangements of orientation selectivity, spatial phase, and retinotopic position across the mature simple-cell layer-more specifically, studies of the arrangement of the spatial positions of ON- and OFF-subregions-could give insight into the interactions underlying the final maps of orientation and of other receptive field properties. This is likely to be a 3-D arrangement, because spatial phase is likely not to have columnar invariance (further discussed below).

This arrangement has thus far been studied only in simple cell pairs recorded on a single electrode. Both the spatial and temporal phase differences of such pairs are evenly distributed over all possible differences (DeAngelis et al., 1992; but see Pollen and Ronner, 1981; Foster et al., 1983). In the model, the spatial phase differences of nearby cells are determined both by their connectivity to one another, and by their simultaneous connectivity to many other cells (e.g., see discussion of systematic phase shifts in Analysis of the model, above). Thus, to compare such data to the model, connectivity information as inferred by cross-correlations as well as systematic mapping across a region of cortex may be needed.

It is possible that the horizontal organization of simple cells may be mediated in part by other cells that do not carry information about spatial phase. If this were the case, the arrangement of simple cell spatial phases might not show a systematic structure or relationship to the orientation map. The anatomical substrate for horizontal synaptic interactions between simple cells within layers 4 and 6 in cat area 17 exists at the appropriate developmental times, as early as the first postnatal week (Callaway and Katz, 1992). However, the substrates for other interactions also exist at this time in cat area 17. The transient neurons of the cortical subplate receive LGN input and participate in microcircuits with layer 4 cells during the first postnatal week (Friaufet al., 1990; Friauf and Shatz, 1991). It is not known whether the responses of these cells preserve spatial phase information. Complex cells lack specificity for spatial phase. Layer 5 complex cells receive inputs from the LGN and layer 4 and make horizontal projections as early as the first postnatal week (Shatz and Luskin, 1986; Callaway and Katy, 1990, 1992). In the young rat, cortical cells are coupled by gap junctions in domains stretching across all cortical layers and extending perhaps $100 \mu \mathrm{m}$ horizontally (Yuste et al., 1992; Peinado et al., 1993). If such domains exist in the developing cat, they could couple simple and complex cells.

\section{Details of cortical maps of orientation and other receptive field properties}

The model provides a mechanism for the development of spatial organization of preferred spatial frequency and of other receptive field properties. Because the model includes only a 2-D cortex, only columnar organization (vertical invariance of receptive field properties) can emerge in simulations. With inclusion of a third dimension, some receptive field properties might develop laminar rather than columnar organization. Furthermore, as discussed in Analysis of the model, above, the structure of cortical maps may vary with nonlinearities, and we do not have an accurate model of biological nonlinearities. Thus, the details of the maps that emerge in the model do not constitute strong predictions. Rather, the robust prediction of the model is that receptive field properties develop some kind of mutually 
coupled spatial clustering and organization, the details of which depend on the details of the intracortical interactions.

The lack of a third dimension provides a specific reason why the model is probably not yet adequate to support detailed conclusions about the cortical organization of preferred orientation. Given a third dimension, connectivity patterns will need to be identified that lead to development of columnar invariance of preferred orientation. There is no reason why spatial phase should then also develop columnar invariance. One can imagine a scenario in which a vertical set of cells, including simple cells with a single preferred orientation but with two or more spatial phases, is established through vertical excitatory and inhibitory interactions, and in which such a set interacts with other such sets at different horizontal locations. Such an interaction between multiple cells of different spatial phases at each cortical "point" may lead to very different predictions for the cortical organization of preferred orientation than the present model, in which there is only a single spatial phase at each cortical point. Development of more realistic, experimentally based models of the early cortical circuitry is critical to further progress in understanding the origins of cortical maps.

\section{Degree of orientation selectivity}

When broader correlation functions are used, cells of both unusually low and unusually high preferred spatial frequency tend to develop poor orientation selectivity. A correlation of low preferred spatial frequencies to poor orientation selectivity has been reported (Tootell et al., 1988; Silverman et al., 1989), but such a correlation of very high preferred spatial frequencies has not previously been suggested. Regions of rapid orientation change also show poor orientation selectivity in the model; biologically, this appears not to be true near orientation pinwheels (Bonhoeffer and Grinvald, 1991; Bartfeld and Grinvald, 1992).

These results do not constitute strong predictions, because the degree of orientation tuning may depend on many details that are not modeled in the present study. As noted previously, developmental nonlinearities could lead cells at orientation boundaries to develop well-oriented receptive fields rather than receptive fields that linearly "mix" neighboring orientations. Similarly, the effects of nonlinearities and of intracortical circuitry in determining a mature cell's response to its inputs could endow sharp orientation tuning on a cell whose receptive field appears only weakly selective in the present model. The timing of input activations can also make a strong contribution to orientation tuning (Ferster, 1987). Thus, in these cells, poor orientation tuning, or late development of sharp orientation tuning, constitute possible outcomes of development under the hypothesis, but probably do not constitute necessary outcomes.

In summary, the strong predictions of the model relate to the spatial structure of simple cell receptive fields, and the mechanisms by which this structure develops and by which developing cells interact to form maps. The model does not make strong predictions about the maps themselves, or about the physiological sharpness of receptive field tuning.

\section{Applicability of the model}

The present theory applies well to systems in which simple cells form in the geniculate-recipient layers. If simple cells form in cortex through projections from unoriented, ON-center or OFFcenter cortical cells, the present theory might also apply, though perhaps with modifications to incorporate feedforward inhibition as well as excitation.

In cats, the vast majority of cells in layer 4 of primary visual cortex are simple cells (Gilbert, 1977; Bullier and Henry, 1979), so the present theory applies well. In macaque monkeys, the magnocellular-recipient layer 4C $\alpha$ of striate cortex contains many oriented cells, most of which are simple cells (Blasdel and Fitzpatrick, 1984; Bullier and Henry, 1980). Thus, the present theory may describe the macaque magnocellular pathway. However, the parvocellular-recipient layers $4 \mathrm{~A}$ amd $4 \mathrm{C} \beta$ contain non-oriented cells (Blasdel and Fitzpatrick, 1984), and it is unclear whether significant numbers of simple cells form further upstream in the parvocellular pathway (Bullier and Henry, 1980; Hubel, 1982). Since this pathway consists of color-selective neurons, it may be necessary to consider competition between the various color-selective neuronal types in order to understand parvocellular development. It should also be noted that the magno- and parvocellular pathways form matching ocular dominance and orientation columns, and so do not develop independently. In other species, such as ferrets (see Chapman, 1991) or tree shrew (see Raczkowski and Fitzpatrick, 1990), the existence or distribution of simple cells has not yet been studied.

\section{Why do model orientation maps resemble experimental maps?}

The structure of the orientation maps that emerge in the model is in many ways arbitrary, as has been emphasized, yet the results resemble experimental maps. Why should this be the case?

If random maps of preferred orientation are band-pass filtered, the resulting periodic but otherwise random orientation patterns closely resemble experimental orientation maps (Rojer and Schwartz, 1990). This likely reflects the fact that a mixture of spatially periodic orientation patterns without a strongly preferred direction (a mixture of Fourier modes from a restricted band of frequencies but many or all possible directions) will produce a continuous, periodic map of orientation with modular iso-orientation regions rather than long iso-orientation rows. Thus, any mechanism that produces a periodic pattern of orientation without a strongly preferred direction is likely to produce maps that resemble actual maps (also discussed in Rojer and Schwartz, 1990; Obermayer et al., 1992; Niebur and Wörgötter, 1993). Experimental maps may have additional structure; for example, differences between cat and monkey maps may in part reflect the difference between an annular power spectrum and a hemicircular one (Fig. 15B). But such differences seem to have only a minor effect on a map's visual appearance compared to the modular structure.

Vortices of orientation of index $\pm 1 / 2$ (points around which orientation cycles by $180^{\circ}$ ) are a natural consequence of such modular maps. An orientation map that is continuous must contain such singularities unless it is topologically equivalent to long iso-orientation rows (Elsdale and Wasoff, 1976; Penrose, 1979). Furthermore, singularities with index \pm 1 (around which orientation cycles by $360^{\circ}$ ) can with a small perturbation of the map be converted to two nearby singularities of index $\pm 1 / 2$. Thus, vortices will be of index $\pm 1 / 2$ except in the unlikely case that two such vortices occur precisely in the same place (sec Swindale, 1982, for a similar argument that vortices will almost always have index $\pm 1 / 2$ ).

Thus, oricntation modules and vortices of index $\pm 1 / 2$ are likely to result from a variety of mechanisms, so production of "re- 
alistic-looking" maps is not a powerful critcria to distinguish models. Given a periodic orientation map, it is the absence, rather than the presence, of such modules and vortices that is surprising, as it implies a preferred direction or other special structure in the orientation map. Such maps with long iso-orientation rows and few or no singularities appear to be found in the tree shrew (Humphrey and Norton, 1980; Humphrey et al., 1980).

\section{Evaluation of the parameter values studied here}

The intracortical interactions studied in the simulations could incorporate excitatory interactions over distances of 200-500 $\mu \mathrm{m}$, as well as inhibitory interactions over farther distances up to about $800 \mu \mathrm{m}$ (using a scale of $92 \mu \mathrm{m} /$ grid interval as in Figs. $14,15)$. Such intracortical influences may be due to diffusion or to synaptic connectivity. Local excitatory influence by diffusion of modulatory substances is not likely to spread more than tens of micrometers, since typical diffusive spread is $1 \mu \mathrm{m}$ in $1 \mathrm{msec}$ and this distance scales as the square root of the time (i.e., $10 \mu \mathrm{m}$ in $100 \mathrm{msec}$ ). If such substances were released simultaneously over much of the dendritic arbor of a single cell, then the $100-150 \mu \mathrm{m}$ radius of such an arbor (Lund et al., 1979) might also play a role. In striate cortex of 2 -weck-old kittens, excitatory but not inhibitory intracortical synaptic connections are seen in studies of cross-correlations, and extend over horizontal distances of up to $600 \mu \mathrm{m}$ (Hata et al., 1993). Intracortical projections within layer 4 of kitten striate cortex extend horizontally many hundreds of micrometers as early as the first postnatal week (Callaway and Katz, 1992). In young rats, cortical cells are coupled by gap junctions over horizontal distances of about $100 \mu \mathrm{m}$ (Yuste et al., 1992; Peinado et al., 1993). The presence of such coupling in other species has not yet been studied; if present, this should represent a purely excitatory coupling. In summary, the purely excitatory intracortical interactions studied in the simulations seem plausible based on available data.

Due to the small amounts of data on young animals, a role for inhibitory interactions in simple cell development cannot be ruled out. Such interactions are observed in studies of adult cat striate cortex. Monosynaptic intracortical synaptic connections, both excitatory and inhibitory, have been shown by studies of cross-correlations to extend horizontally about $650 \mu \mathrm{m}$ in all layers of cat striate cortex, with the most frequent connections extending less than $400 \mu \mathrm{m}$ (Hata et al., 1991); periodic longer-range connections also exist (Gilbert et al., 1990). Chemical excitation of cells at one location in cat visual cortex produces inhibition in cells from 100 to $500 \mu \mathrm{m}$ horizontally distant (Hess et al., 1975). Chemical inhibition of cells at one location can lead to either loss of excitation or disinhibition of horizontally distant cells, suggesting inhibition at distances of about 500 $\mu \mathrm{m}$ and a mixture of inhibition and excitation at distances of a millimeter or more (Crook et al., 1991; Wörgötter and Eysel, 1991 b). Spatiotemporal patterns of adult cortical activity appear to have characteristic spatial oscillations with a period of around $1 \mathrm{~mm}$ (Shoham et al., 1991), consistent with intracortical interactions with a similar Fourier transform peak, for example, excitation over a radius of about $200 \mu \mathrm{m}$ and longer-range inhibition. The mixed excitatory/inhibitory functions studied here could incorporate the submillimeter interactions that have been observed in adult cat cortex.

To estimate the correlation function $C^{1)}$, I use data from the adult cat (see further discussion below of measurements of correlations during development). These suggest plausible, though not compelling, parameter values for the model. I assume, as in Results, that $C^{\text {D }}$ follows the correlation function measured in the mature cat retina, but extends to farther distances with a change in sign. At $10^{\circ}$ eccentricity, adult retinal $X$-cell correlations go to zero at about $0.5^{\circ}$ separation (Mastronarde. $1983 \mathrm{a}, \mathrm{b})$. The cortical magnification factor there is about 650 $\mu \mathrm{m} /$ degree (Tusa et al., 1978), so $0.5^{\circ}$ corresponds to about 325 $\mu \mathrm{m}$ across cortex. Geniculocortical X-cells in the adult cat typically arborize over a radius of 500-750 $\mu \mathrm{m}$ (Humphrey et al. 1985). Layer 4 spiny stellate cells have dendritic arborizations of radius 100-150 $\mu \mathrm{m}$ (Lund et al., 1979). Assuming the arbor function corresponds to the overlap of these two arborizations, the zero-crossing of $(D$ occurs at about $40-50 \%$ of an arbor radius, yielding an estimated mean preferred spatial frequency of about $1-1.25$ cycles per arbor diameter.

Thus, the estimated size of $\left(^{17}\right.$ relative to an arbor, based on measurements in adult cat retina, is within the range that leads to good orientation selectivity in the present model. However, using $92 \mu \mathrm{m} /$ grid interval as in Figures 14 and 15 , the absolute sizes of both $C^{(1)}$ and the arbor are about $20-40 \%$ smaller in the simulations than in the estimates from adult cats. This should have little effect on results. A concurrent change in arbor and correlation sizes causes little change in receptive field structure, and in simulations leads to little change in the spatial period of cortical organization of preferred orientation (Fig. 14).

\section{Evaluation in light of experimental studies of development}

The theory presented here implies that the earliest developing orientation-selective cortical cells should be simple cells in the layers that receive unoriented $\mathrm{ON}$-center and OFF-center input, and that these cells should initially receive spatially mixed ON/ OFF input. The theory also assumes that initial orientation selectivity evolves without the influence of patterned visual input.

Significant numbers of oriented cells are found in kitten V 1 as early as visual responses can be found, ranging from P6 (postnatal day 6) to P12 in various studies (Hubel and Wiesel, 1963; Blakemore and van Sluyters, 1975; Buisseret and Imbert, 1976; Fregnac and Imbert, 1978; Bonds, 1979; Tsumoto and Suda, 1982; Albus and Wolf, 1984; Braastad and Heggelund, 1985). Most of these studies occurred before the age of natural eye opening, so these oriented cells develop without vision. In kittens before P14, nearly all oriented cells have been reported to be simple cells in deep layers of cortex (Blakemore and van Sluyters, 1975; Albus and Wolf, 1984), and to be largely or exclusively found in the adult geniculate-recipient layers 4 and 6 (Albus and Wolf, 1984) (but see Tsumoto and Suda, 1982). Others report encountering both simple and complex cells in the youngest kittens (Hubel and Wiesel, 1963; Tsumoto and Suda, 1982; Braastad and Heggelund, 1985).

Albus and Wolf (1984) reported a class of cells, many of which were not orientation selective, with linear spatial summation of responses but spatially commingled ON- and OFF-responses. These were found only in layer 4 before about P18; layer 4 eventually consists largely or exclusively of simple cells (Gilbert, 1977; Bullier and Henry, 1979). The early presence of such cells and their disappearance over the period that orientation selectivity develops strongly suggest that $\mathrm{ON} / \mathrm{OHF}$ segregation in simple cell receptive fields can evolve from an initially unseg- 
regated state. Some investigators might have regarded these cells as complex cells; this provides one possible resolution to the conflicting reports from young kittens.

A possible inconsistency between these studies of cat development and the present model is the report of Albus and Wolf (1984) that most $(76 \%)$ visually responsive cells in very young kittens have only OFF-responses, with ON-responses becoming equal to OFF only by about P20. Braastad and Heggelund (1985) agree that $O F F$-responses are on average stronger than $O N$ before 3 weeks of age, but report finding some ON-response in virtually all cortical receptive fields. They attribute the difference to their use of stimulus protocols that enhance detection of the weaker ON-responses. If their results are accepted, then the main problem for the present theory is that it ignores an early ON/OFF incquality, perhaps due to the greater dark activity of OFF-inputs (Mastronarde, 1983b), that leads to more rapid development of OFF-responses in cortex. This is unlikely to lead to fundamental changes in the conclusions because, given the basic hypothesis as to the structure of correlations, it should not substantially change the spatial period of ON/OFF alternation of a best-correlated set of inputs to a cortical cell.

Results in other species also appear consistent with the model, although fewer data are available. Normal, adult-like tuning and organization of orientation selectivity exist at birth in monkey V1 (Wiesel and Hubel, 1974) and in sheep V2 (Ramachandran et al., 1977). Simple cells in newborn monkey are reported to be found primarily in layer 4 and to have segregated ON- and OFF-subregions (Hubel and Wiesel, 1968). In ferret (Chapman, 1991), some orientation selectivity is found as early as visual responses can be recorded (P23). Normal maturation of orientation selectivity in ferret depends on neural activity, but progresses normally under binocular lid suture in all but deep layers. suggesting that spontaneous activity may be sufficient for simple cell maturation.

\section{Evaluation in light of experimental data on input correlations}

Two types of dark correlations have been measured experimentally in the visual system of various species. In the mature retinas of cats, rabbits, and goldfish, ON cells are correlated with other ON cells, and OFF cells with other OFF cells, at retinotopic separations such that their receptive field centers significantly overlap, while ON cells are anticorrelated with OFF cells at the same separations (Arnett, 1978; Arnett and Spraker, 1981; Mastronarde, 1983a,b). At larger separations in rabbits and goldfish, no correlations were detected; this has not been studied in cat dark correlations. Correlations in the mature cat LGN between cells whose centers overlap are similar to those in retina, except that asymmetric correlations (e.g., one cell tending to fire some milliseconds before another) are also observed (Arnett, 1975; Stevens and Gerstein, 1976). LGN correlations have not been studied at larger separations where one cell's center overlaps another's surround.

In fetal or early neonatal retinas, a different pattern of activity is seen in which retinal ganglion cells fire in bursts a few seconds in duration, separated by minutes of silence (Masland, 1977; Maffei and Galli-Resta, 1990; Meister et al., 1991). In ferret or kitten retina in vitro, this activity is organized spatially as oriented traveling waves of correlated bursting (Meister et al., 1991). In ferret retina, this activity was observed as late as P22, and is no longer present at P30 (Wong et al., 1991). In cat retina, it was observed as late as P1 (Meister et al., 1991). Retinal ganglion cells could not be visually driven at the ages at which wave activity was observed (M. Meister, personal communication). In comparison, as discussed previously, visual responses in ferret cortex first appear at $\mathrm{P} 23$, and the earliest recordings from cat cortex are from P6. In both cat and ferret, these earliest cortical recordings include orientation-selective responses, although in ferret the major development of orientation selectivity occurs several weeks later (Chapman, 1991). Thus, the wavelike, bursting pattern of activity disappears at roughly the time that visually driveable retinal circuitry and orientation selectivity may first arise. The waves may gradually diminish in preceding weeks (Meister, personal communication), so it is conceivable that there is a gradual, overlapping transition between correlation structures.

There are several reasons why the early, wave-like activity seems unlikely to underlie simple cell development. First, the wave-like activity may disappear too early to play this role, although this remains unclear. Second, no distinction between $\mathrm{ON}$ - and OFF-center populations has been discovered in this activity ( $R$. Wong and M. Meister, personal communication). That is, although ganglion cells were not visually driveable and so could not be classified as ON- or OFF-center, the ganglion cell population was not observed to separate into distinct groups that tended to fire on separate rhythms. ON and OFF retinal inputs to the LGN segregate into laminae in an activity-dependent manner between P14 and P21 in ferret (Hahm et al.. 1991), so some distinction in the activities of $\mathrm{ON}$ - and OFF-inputs is likely to exist in vivo at that time. Unless such a distinction is discovered in the waves of activity, they would not appear to be the correlation structure guiding ON/OFF segregation in LGN or cortex.

Third, the waves are very wide compared to geniculocortical arbors and simple cell receptive fields. Correlations between the activities of cell pairs in these early retinas decrease slowly with the distance between two cells, decreasing by only a factor of 2 over a separation of about 10-20 retinal ganglion cell (RGC) spacings (Meister, personal communication). That is, the full width at half height of the correlation function is $20-40 \mathrm{RGC}$ spacings. In contrast, geniculocortical arbors have a radius corresponding to 4-12 RGC spacings; the largest subregion of cat simple cell receptive fields corresponds to 5-20 RGC spacings; and the width of the entire receptive field is 9-40 RGC spacings [in adult cat at $10-12.5^{\circ}$ eccentricity: $1^{\circ}$ corresponds to between 5 and 10 RGC spacings (Mastronarde 1983a,b); cortical magnification factor is about $650 \mu \mathrm{m} /$ degree (Tusa et al., 1978); $\mathrm{X}$-cell arbors have a radius of 500-750 $\mu \mathrm{m}$ (Humphrey et al., 1985 ), or $0.75-1.15^{\circ}$; largest subregion is $1.67 \pm 0.63^{\circ}$, and width of receptive field is $2.88 \pm 1.04^{\circ}$ (Heggelund, 1986)]. Thus, the waves are so wide that they should simultaneously activate all geniculate inputs to a single cortical cell, and so would not appear "oriented" for purposes of competition on a single cell. They appear too large to account for the development of simple cell subregions, and are probably too large even to account for the entire width of the largest simple cell receptive fields.

In contrast to the early wave-like activity, the mature retinal and geniculate dark activity distinguishes $\mathrm{ON}$ - and OFF-center cells and, as shown in Figure 13, has length scales appropriate to account for simple cell receptive field structure. The major problems for the hypothesis that the mature activity underlies simple cell development are (1) the uncertainty whether it exists sufficiently early and (2) the lack of any "Mexican hat" structure in measurements thus far: correlations appear to go to zero, rather than changing sign, at distances where receptive field 
centers no longer overlap. The absence of a Mexican hat structure in retinal measurements is actually in accord with the theory: retinal inputs segregate hetween postsynaptic cells in the LGN, so the theory predicts that retinal correlations either lack a Mexican hat structure, or else have such a structure but with sign change at a separation wider than a retinogeniculate arbor radius.

The LGN might acquire a Mexican hat structure in two ways. First, if a Mexican hat structure is absent in retinal dark activity, it could be induced in the LGN by intrinsic inhibitory circuitry (c.g., Sillito and Kemp, 1983; Kaplan et al., 1987; Mastronarde, 1987; Lindstrom and Wrobel, 1990; Nelson, 1991) and/or by the stronger dark surrounds of LGN receptive fields relative to retinal receptive fields (Virsu et al., 1977; but see Kaplan et al., 1979). Second, if a Mexican hat structure is present but is thus far undetected in retinal dark activity, and its sign change occurs outside the narrow radius of retinogeniculate arbors (Sretavan and Shaty, 1987), the sign change might nonetheless occur within the broader radius of geniculocortical arbors.

\section{Possible effects of orientation bias in input receptive fields}

Retinal and geniculate receptive fields are slightly clliptical rather than circular (Levick and Thibos. 1982; Albus et al., 1983; Leventhal and Schall, 1983; Soodak et al., 1987). It has been proposed that this orientation bias may contribute to the dcvelopment of cortical orientation selectivity (Albus et al., 1983; Schall et al., 1986). In support of this, it has been reported that the mature distributions with eccentricity of retinal preferred orientations and cortical preferred orientations are similar (Schall et al., 1986).

Under the present model, development is determined by the correlation functions. The degree of correlation in dark activity of retinal ganglion $X$-cells appears to match closely the overlap of their dendritic fields (Mastronarde, 1983a,b). The dendritic fields of retinal ganglion cells are reported to have elliptical biases, with a similar distribution across the retina as the biases of receptive fields (Leventhal and Schall, 1983; Schall et al., 1986). Elliptical bias in the dendritic fields would lead to bias in their overlaps, which could produce bias in the correlation functions. Thus, elliptical bias in the receptive fields may be accompanied by a similar bias in the correlation functions.

Such bias in the correlation functions could not account for the development of orientation selectivity, because (1) development with a moderately elliptical but positive correlation function does not lead to development of oriented arrangements of afferents (not shown), while (2) ON/OFF competition with an unbiased Mexican hat correlation function does lead to orientation selectivity, as shown here. However, such bias could explain the reported correspondence between the distributions of retinal and cortical preferred orientations. For example, if there were a larger proportion of radial dendritic fields in one portion of the retina, this could lead retinal and corresponding LGN neurons to be correlated at slightly longer distances in the radial direction than in the tangential, and this could bias the cortical competition to slightly favor radial preferred orientations.

\section{Evaluation in light of experimental data on the mature} cortex

\section{Convergence of geniculate inputs onto simple cells}

The present model requires that multiple LGN inputs, including both ON-center and OFF-center inputs, converge onto individ- ual simple cells, in a manner corresponding to the simple cell's subregions. Convergence of multiple geniculate inputs onto single simple cells in cats has been demonstrated in several studies (Tanaka, 1983; Freund et al., 1985; Ferster, 1987). Tanaka (1983) directly demonstrated convergence of both ON-and OFF-center LGN inputs onto a single cat cortical simple cell. Bullier et al. (1982) concluded by less direct methods that about $75 \%$ of layer 4 simple cells in the cat receive both ON-center and OFF-center LGN input. This roughly agrees with findings that only about $20 \%$ of simple cells in the cat (Palmer and Davis, 1981; Mullikan et al., 1984b) and 27\% in monkey (Schiller et al., 1976) are unimodal, that is, have only one excitatory subregion, either ON or OFF (but see Heggelund, 1986, who finds far fewer unimodal cells in cats). Tanaka (1983) found that the surrounds of LGN cells do not contribute to the visual responses of nonunimodal simple cells, also suggesting that such cells are driven by both ON-center and OFF-center geniculate inputs.

Tanaka (1983) showed that in cat, more than one-third of ON-geniculate $X$-cells with receptive field center overlapping an ON-subregion of an area 17 simple cell, or OFF overlapping with OFF, made a monosynaptic connection onto the simple cell. This high rate of connection is suggestive that inputs converge in a manner that corresponds to the simple cell's subregions, although no data exist on the rate of connection (if any) for geniculate cells whose centers overlap the opposite subregion type. That the centers of most ON-center geniculate inputs overlap ON-subregions, and OFF overlap OFF, is also suggested by the preservation of the spatial structure of OFF-subregions of cat simple cells after ON-center inputs to cortex are silenced (Sherk and Horton, 1984), and by the fact that simple cell subregion structure is present in both the pattern of excitatory input and the pattern of inhibitory input received by the cell (Ferster, 1988).

The development of unimodal cells might be accounted for in the present model in several ways. First, sufficient decrease of receptive field size during development leads some initially bimodal cells to become unimodal (Fig. 10A). Consistent with this idea, in monkeys (Schiller et al., 1976) and cats (Palmer and Davis, 1981; Mullikan et al., 1984a,b), bimodal cells have receptive fields about twice as wide as unimodal cells. This suggests that subregions in either case are formed by a common competitive mechanism. Second, biological variation in connectivity or correlations might lead to a subpopulation of cells for which a change in sign of the correlation function occurs at a distance of an arbor radius or more. Such a subpopulation would in isolation develop unimodal, unoriented receptive fields. Interaction with cells developing bimodal, oriented receptive fields could lead unimodal cells to develop an oriented set of inputs, and/or endow orientation selectivity via intracortical connections. Consistent with this idea, cat unimodal cells appear to be a specific subpopulation, consisting of inhibitory cells in the deep parts of layer 4 and in layer 6 (Toyama et al., 1981; Mullikan et al., 1984a).

\section{Mature degree of orientation tuning}

The present model accounts for the development of "HubelWiesel" receptive fields. These have been shown adequate to yield mature orientation tuning, provided a more realistic model of cortical response is used (Ferster, 1987). Intracortical inhibition may enhance this tuning (Wörgötter and Koch, 1991). As discussed in Methods, the simple linear model of cortical cell response used here to assess selectivity does not yield mature 
orientation tuning, but this is a problem of the response model rather than of the developmental model.

\section{Role of $O N$ - and $O F F$-center input streams in mature orientation selectivity}

After selective silencing of ON-center inputs in the retina of the mature animal, cortical cells in both cats (Sherk and Horton, 1984) and monkeys (Schiller, 1982) retain normal orientation tuning. The present hypothesis states that competition between ON-center and OFF-center inputs is necessary for the development of orientation selectivity, but not for its mature expression. Ferster (1987) showed that a Hubel-Wiesel model of simple cell receptive fields retains normal orientation tuning after $\mathrm{ON}$-center inputs are silenced.

\section{Other models for the development of orientation selectivity}

Models for the development of orientation selectivity may be roughly divided into two classes. One class, including the present model, accounts for the development of individual oriented receptive fields. In these models the fundamental variables are the synaptic strengths of topographically organized inputs; orientation-selective receptive fields emerge out of the strengthening and weakening of synapses. The second class of models assumes as fundamental variables a cell's preferred orientation and degree of orientation selectivity, and studies the organization of cortical maps. I will discuss each class of models in turn.

\section{Models that account for the development of oriented receptive} fields

A model for the activity-dependent development of orientation selectivity was first proposed by von der Malsburg (1973). The model was similar to the present one in using a simple Hebbian plasticity rule and a $2-\mathrm{D}$ cortex with distance-dependent connectivity, and in using conservation of synaptic strength over each postsynaptic cell to achieve a competitive outcome. The model differed from the present one in using only a single type of input, with full connectivity between input cells and cortical cells, and in using as input patterns oriented bars of various orientations, each flashed at the center of the input grid. Training on these patterns led cortical cells to develop oriented receptive fields that varied only in preferred orientation, not in spatial phase (the oriented set of connections to a cortical cell always ran through the middle of the input grid). Nearby cells tended to develop similar preferred orientations. This model would not explain the segregation of ON- and OFF-inputs within the receptive field, and did not address development of orientation selectivity in the absence of vision or the development of spatial phase variation. A related model was proposed by Perez et al. (1975).

A scenario like that studied here, in which a circularly symmetric, spatially oscillating correlation function leads to the development of orientation-selective receptive fields and to segregation of inputs within receptive fields, was first demonstrated by Linsker (1986a-c) (analyzed in MacKay and Miller, 1990a,b). There are three major differences between Linsker's model and the present one. First, Linsker postulated feedforward synapses that could take either positive or negative strengths, rather than ON-center and OFF-center populations of purely excitatory inputs. This is biologically different from the present model, and also mathematically different: Linsker's equations would be fundamentally altered by the inclusion of nonlinearities, whereas the early development of the present model is independent of many nonlinearities (Miller, 1990c). Second, Linsker used a constraint fixing the percentages of positive and negative synapses in the final receptive field of a cell. Applied to the present model, this would be equivalent to fixing the percentage of $\mathrm{ON}$ center versus OFF-center synaptic input in each cortical receptive field. Orientation-selective receptive fields developed in his model only in a narrow, tightly tuned regime of the constraint parameter, and then only in the late, nonlinear stage of development (MacKay and Miller, 1990a).

Third, receptive field development was studied only in single, isolated cortical cells. The cortical organization of preferred orientation was studied only in the case that all cortical receptive fields are identical except for preferred orientation; that is, all have identical spatial phase and preferred spatial frequency. If spatial phase cannot vary, then the argument of Figure 16 is altered by the elimination of Figure $16 B$, so the nature of cortical organization differs from the present model. This led to the prediction that vertically oriented cells should be found in isoorientation patches that are elongated in the retinotopically vertical direction across the cortex, and horizontally oriented cells should be similarly found in patches extended in the retinotopically horizontal direction. This prediction disagrees with existing experimental results (Blasdel and Salama, 1986; Swindale et al., 1987; Diao et al., 1990).

Tanaka (1992) has independently proposed a relationship between $\mathrm{ON} / \mathrm{OFF}$ competition and the development of orientation selectivity. His model is formally equivalent to the present model with one crucial difference: in his model, each cortical cell eventually receives only one $L G N$ input. Tanaka defines cortical receptive fields as the convolution of the input arrangement with the intracortical interaction function. This means that a cortical cell's receptive field is due to its single input from the LGN. plus its input from all other cortical cells within reach of the intracortical interaction function. Oriented receptive fields arise if this convolution is not circularly symmetric. For example, if $\mathrm{ON}-$ and OFF-inputs at least partially segregate into cortical patches like ocular dominance patches, then oriented cells will arise. ON-inputs contribute positively and OFF-inputs contribute negatively to the convolution; this means that an "ON" subregion may show both ON- and OFF-responses to light, although ON-responses will be stronger (and similarly for "OFF" subregions).

In Tanaka's model, orientation selectivity arises from the breaking of circular symmetry in the pattern of inputs to different cortical cells, rather than to individual cortical cells. This mechanism of symmetry-breaking leads to different requirements for the development of orientation selectivity than the present model: ocular-dominance-like patchiness of the inputs develops when there is a Mexican hat structure in the intracortical interactions, and does not require such a structure in the input correlation function. Under this mechanism, at least some cortical cells must initially respond to their nonoriented LGN input before oriented responses could appear. In ferrets, which have a patchy arrangement of ON and OFF LGN inputs (7ahs and Stryker, 1988), there are many nonoriented cells in layer 4 (Chapman, 1991) that could potentially provide such early responses.

Soodak (1987, 1991, and unpublished observations) proposed a model for the development of orientation selectivity based on the following rule: inputs whose responses are temporally in phase with the cortical cell's response are weakened, while those 
$180^{\circ}$ out of phase are strengthened and those at $90^{\circ}$ are unchanged. This rule is derived most casily from an anti-Hebbian plasticity rule (a rule in which temporal correlation of pre- and postsynaptic activity leads to synaptic weakening). Soodak assumed that an ON- and an OFF-center input have equal and opposite responses to an identical stimulus. Competition under this rule in the absence of patterned visual input then leads to an exact balancing of the strength of ON-center and OFF-center inputs to each cortical cell. In analogy to electrostatics, this sets the ON/OFF "charge" (the total ON-center strength minus the total OFF-center strength in the receptive field) to zero. This leaves only an ON/OFF "dipole" and higher-order ("quadrupole," etc.) moments, which represent spatial inhomogeneities in the distribution of the balanced ON- and OFF-center inputs within the receptive tield. The "dipole moment" has an orientation-selective response. As the "charge" is climinated, the dominant response becomes that due to the dipole and so the cell's responses become orientation selective.

A virtuc of this model is that it depends only on the local anticorrelation of $\mathrm{ON}$ - and OFF-center inputs, which has been observed; a "Mexican hat" correlation structure, which has not yet been observed, is not required. However, several problems exist. The mechanism does not yield spatial segregation of $O N$ center and OFF-center inputs within the receptive field, but rather yiclds a spatial mixture of inputs with slight spatial imbalances. It is unclear whether periodic, segregated subregions could nonetheless emerge, for example, through cancellation of the balanced ON-center and OFF-center afferent drives and through intracortical interactions. If average positive afferent responses are sufficiently stronger than average negative responses (e.g., Ferster, 1987), then all regions of the final receptive field will give positive responses to all stimuli, so orientation selectivity will not result. If the "dipole" input is small relative to the input provided by a single afferent, orientation tuning would be eliminated by realistic levels of variance in the afferent activities. Orientation tuning and the spatial structure of OFFsubregions both would be likely to disappear if ON-center inputs are silenced, but biologically this does not occur (Schiller, 1982; Sherk and Horton, 1984).

\section{Models that treat orientation preferences as fundamental variables}

Simplified models of cortical map formation characterize receptive fields and the interactions between them by a reduced set of variables, such as preferred orientation and orientation selectivity, in addition to retinotopic position. A distance-dependent "Mcxican hat" interaction in cortex, by which each cell influences nearby cells to develop receptive fields similar to its own, and influences more distant cells to develop receptive fields opposite to its own, then leads to a periodic pattern of the reduced variables (note: the present model depends on a Mexican hat in the input correlations, not in cortex). Such a model for the development of the periodic arrangement of cortical preferred orientations and the enhancement of orientation selectivity was proposed by Swindale (1982). Models that similarly depend on a spatially Mexican hat interaction have becn applicd to many examples of pattern formation, such as zebra stripes and leopard spots (Turing. 1952; Murray, 1989). A Mexican hat interaction acts as a band-pass filter in Fourier space, and therefore leads to the growth of a spatially periodic pattern of the reduced variables with wavelength corresponding approximately to the peak of the Fourier transform of the Mexican hat function. Direct application of a band-pass filter to a random pattern can similarly create a periodic pattern like that found biologically (Rojer and Schwart7, 1990).

Such reduced models of cortex usually depend on the ad hoc assumption that the reduced variables and a simple interaction between them are sufficient to describe cortical development. There are two problems with this assumption. First, it does not describe receptive field development, for example, the conditions under which receptive fields become characterized by their orientation tuning. Second, it may be unwarranted. In the present model, the interaction between two receptive fields cannot be described in terms of the reduced variables of orientation preference and selectivity alone, but depends critically on spatial phase (Fig. 16). An approximate reduced description can be derived that utilizes spatial phase and spatial frequency in addition to orientation as reduced variables, but the resulting interaction has a complicated structure (K. D. Miller, unpublished observations).

Recent models using the "self-organizing feature map" (Obermayer et al., 1990, 1992) or "elastic net" (Durbin and Mitchison, 1990) algorithms represent an intermediate level of complexity. These models use a reduced set of variables to describe receptive fields and input activity patterns, but use a Hebb-type rule to describe development. Certain abstractions are used to describe cortical cell activation. These models lead to locally continuous mappings in which a constant distance across the cortex corresponds to a roughly constant distance in the reduced "input space." If the input space has more than the cortex's two dimensions - for example, the 5-D space of 2-D retinotopic position, orientation preference and selectivity, and ocular dominance-this means that, when one feature is changing rapidly across cortex, the others will be changing slowly. For this reason, the models predict that orientation changes rapidly where retinotopy changes slowly, and vice versa.

This prediction may arise for mathematical reasons similar to those underlying the present prediction that the spatial phase and preferred orientation of receptive fields should systematically covary: for a reduced model in which inputs are of a single type and receptive fields have only a single excitatory subregion, a shift in spatial phase is identical to a shift in retinotopic position. Experimentally, however, these predictions are quite different. These models similarly predict covariation of preferred orientation and ocular dominance, and this accounts successfully for observed interactions between the two columnar systems in monkeys (Obermayer et al., 1992). Simultaneous development of orientation and ocular dominance has not been studied with the present model. As implemented thus far, these models do not allow prediction of the dependence of emergent spatial scales on biologically identifiable parameters, cannot break symmetry to develop oriented responses from nonoriented input patterns, and do not include ON- and OFF-center inputs or account for their segregation within simple cell receptive fields. But they demonstrate simple rules that are formally similar to Hebbian rules and that can account for complicated aspects of cortical maps, and so may provide a guide for future work.

\section{Conclusion: competitive mechanisms and neural structure}

In a variety of systems, where two input populations converge on a single postsynaptic target, the two input types segregate between postsynaptic cells. It has not previously been appreciated that the segregation of ON-and OFF-center inputs within receptive fields of cortical simple cells might reflect a similar 
competitive process, but in a different parameter regime. Perhaps the strongest argument in favor of the present model is that it naturally explains this segregation, while also accounting for the development and basic organization of orientation selectivity.

The connectivity patterns that result from synaptic competition might be functional, specifically selected for by evolution. Alternatively, such patterns may be selectively neutral, and arise as epiphenomena of competitive rules that have evolved for other reasons. For example, in the frog, if two eyes are artificially forced to innervate a single tectum, ocular dominance stripes form (Constantine-Paton et al., 1990); this presumably reflects the action of rules that evolved without regard for ocular dominance, since in nature the frog's two eyes never innervate a common tectum. A third possibility is that an observed pattern might be one of a broad variety that can equally well be used to construct a functional visual system. Thus, ON- and OFFcenter cells in the LGN are segregated in separate laminae in mink (LeVay and McConnell, 1982), ferret (Stryker and Zahs, 1983), and tree shrew (Conway and Schiller, 1983), and in the parvocellular layers in rhesus monkey (Schiller and Malpeli, 1978), but there is at most incomplete laminar segregation in cat (Bowling and Caverhill, 1989) and no obvious segregation in monkey magnocellular layers (Schiller and Malpeli, 1978). $\mathrm{ON}$ - and OFF-inputs to cortex show laminar segregation in tree shrew (Raczkowski and Fitzpatrick, 1990) but column-like segregation in mink (McConnell and LeVay, 1984) and ferret (Zahs and Stryker, 1988). Cortical ocular dominance segregation is columnar in the cat and in some species of monkey, but there is at most incomplete segregation in other species of monkey (revicwed in LcVay and Nelson, 1991) and only a partial, laminar segregation in the tree shrew (Raczkowski and Fitzpatrick, 1990). Such variety suggests that the details of these patterns might not be functionally important, although alternatively they might represent functional specializations. The present model, by bringing segregation within simple cell receptive fields and development of orientation selectivity within the common framework of synaptic competition, raises the intriguing possibility that these also might not be central features of the design of the visual system, but rather could arise as one out of many equally usable possible arrangements, or even as epiphenomena of synaptic competition [the sharpening of orientation selectivity by intracortical connectivity, which might suggest design, can emerge out of very general properties of cortical connectivity (Wörgötter and Koch, 1991)].

In the future, the present framework can be extended in several ways. Study of simultaneous competition between $\mathrm{ON}$ - and OFF-center inputs from the two eyes may reveal emergent relationships between ocular dominance, orientation, and disparity tuning. Study of a 3-D cortex and of specific connectivity between separate excitatory and inhibitory cortical populations seems necessary to understand the interacting cortical organizations of spatial phase, spatial frequency, and preferred orientation. Consideration of temporal structure in subcortical and cortical responses may allow an understanding of the development of spatiotemporal receptive fields. The model may he deepened or altered by incorporation of greater biophysical detail, plasticity of intracortical synapses, or additional possible mechanisms of plasticity such as sprouting and retraction of axonal terminals.

The present study, in combination with previous studies (Miller et al., 1989), demonstrates that simple mechanisms of cor- relation-based synaptic plasticity are adequate to account for a number of the most striking features of visual cortex. It seems possible that such mechanisms, when combined with greater realism in models of the input populations and of the cortical circuitry, may ultimately prove adequate to account in some detail for many of the rich and varied structures found in thalamic and early cortical processing.

\section{References}

Albus K, Wolf W (1984) Early post-natal development of ncuronal function in the kitten's visual cortex: a laminar analysis. J Physiol (Lond) 348:153-185.

Albus K, Wolf W, Beckman R (1983) Orientation bias in the response of kitten LGNd neurons to moving light bars. Dev Brain Res 6:308313

Arnett DW (1975) Correlation analysis of units recorded in the cat dorsal lateral geniculate nucleus. Exp Brain Res 24:111-130.

Arnett DW (1978) Statistical dependence between neighboring retinal ganglion cells in goldfish. Exp Brain Res 32:49-53.

Arnett DW, Spraker TE (1981) Cross-correlation analysis of the maintained discharge of rabbit retinal ganglion cells. J Physiol (Lond) 317 $29-47$.

Barlow HB, Levick WR (1976) Threshold setting by the surround of cat retinal ganglion cells. J Physiol (Lond) 259:737-757.

Bartfeld E, Grinvald A (1992) Relationships between orientation-preference pinwheels, cytochrome oxidase blobs, and ocular-dominance columns in primate striate cortex. Proc Natl Acad Sci USA 89:1190511909.

Bienenstock FI, Cooper I.N Munro PW (1982) Theory for the development of neuron selectivity: orientation specificity and binocular interaction in visual cortex. J Neurosci 2:32-48.

Birkhoff G, Rota G-C (1978) Ordinary differential equations, 3d ed. New York: Wiley.

Blakemore C, van Sluyters RC (1975) Innate and environmental factors in the development of the kitten's visual cortex. J Physiol (Lond) 248:663-716.

Blasdel GG (1992) Orientation selectivity, preference and continuity in monkey striate cortex. $J$ Neurosci 12:3139-3161.

Blasdel GG, Fitzpatrick D (1984) Physiological organization of layer 4 in macaque striate cortex. J Neurosci 4:880-895.

Blasdel GG, Salama G (1986) Voltage-sensitive dyes reveal a modular organization in monkey striate cortex. Nature 321:579-585.

Bliss TVP, Collingridge GL (1993) A synaptic model of memory: longterm potentiation in the hippocampus. Nature 361:31-39.

Bonds AB (1979) Development of orientation tuning in the visual cortex of kittens. In: Developmental neurobiology of vision (Freeman RD, ed), pp 31-41. New York: Plenum.

Bonhoeffer T, Grinvald A (1991) Iso-orientation domains in cat visual cortex are arranged in pinwheel-like patterns. Nature 353:429-431.

Bowling DB, Caverhill JJ (1989) On/Off organization in the cat lateral geniculate nucleus: sublaminae vs. columns. J Comp Neurol 283:161168

Braastad BO, Heggelund P (1985) Development of spatial receptivefield organization and orientation selectivity in kitten striate cortex. J Neurophysiol 53:1158-1178.

Buisseret P, Imbert M (1976) Visual cortical cells: their developmental properties in normal and dark-reared kittens. I Physiol (Lond) 255: $511-525$.

Buisseret P, Gary-Bobo E, Milleret C (1988) Development of the kitten visual cortex depends on the relationship between the plane of eye movements and visual inputs. Exp Brain Res 72:83-94.

Bullier J, Henry GH (1979) Laminar distribution of first-order neurons and afferent terminals in cat striate cortex. J Neurophysiol 42:12711281.

Bulier J, Henry GH (1980) Ordinal position and afferent input of neurons in monkey striate cortex. J Comp Neurol 193:913-935.

Bullier J, Mustari MJ, Henry GH (1982) Receptive-field transformations between LGN neurons and S-cells of cat striate cortex. $\mathrm{J}$ Neurophysiol 47:417-438.

Callaway EM, Katz LC (1990) Emergence and refinement of clustered horizontal connections in cat striate cortex. J Neurosci 10:1134-1153.

Callaway EM, Katz LC (1992) Development of axonal arbors of layer 4 spiny neurons in cat striate cortex. J Neurosci 12:570-582. 
Chapman B (1991) Studies of primary visual cortex and its development in cat and ferret. PhD thesis, University of California San Francisco.

Chapman B, Zahs KR, Stryker MP (1991) Relation of cortical cell orientation selectivity to alignment of receptive fields of the geniculocortical afferents that arborize within a single orientation column in ferret visual cortex. J Neurosci 11:1347-1358.

Constantine-Paton M. Cline HT, Debski E (1990) Patterned activity, synaptic convergence and the NMDA receptor in developing visual pathways. Annu Rev Neurosci 13:129-154.

Conway JL, Schiller PH (1983) Laminar organization of tree shrew dorsal lateral geniculate nucleus. J Neurophysiol 50:1330-1342.

Crook JM, Eysel UT, Machemer HF (1991) Influence of GABA-induced remote inactivation on the orientation tuning of cells in area 18 of feline visual cortex: a comparison with area 17 . Neuroscience 40:1-12.

DeAngelis GC, Ghose GM, Ohzawa I, Freeman RD (1992) Spatiotemporal receptive field structure and phase relationships between adjacent simple cells in the cat's striate cortex. Soc Neurosci Abstr $18: 10$.

Diao Y-C, Jia W-G, Swindale NV, Cynader MS (1990) Functional organization of the cortical $17 / 18$ border region in the cat. Exp Brain Res 79:271-282.

Dubin MW, Stark LA, Archer SM (1986) A role for action-potential activity in the development of neuronal connections in the kitten retinogeniculate pathway. J Neurosci 6:1021-1036.

Durbin R, Mitchison G (1990) A dimension reduction framework for understanding cortical maps. Nature 343:644-647.

Elsdale T, Wasoff F (1976) Fibroblast culturcs and dermatoglyphics: the topology of two planar patterns. Rouxs Arch 180:121-147.

Enroth-Cugell C, Lennie P (1975) The control of retinal ganglion cell discharge by receptive field surrounds. J Physiol (Lond) 247:551-578.

Ferster D (1987) Origin of orientation-selective epsps in simple cells of cat visual cortex. J Neurosci 7:1780-1791.

Ferster D (1988) Spatially opponent excitation and inhibition in simple cells of the cat visual cortex. J Neurosci 8:1172-1180.

Ferster D (1990a) X-and Y-mediated synaptic potentials in neurons of areas 17 and 18 of cat visual cortex. Vis Neurosci 4:115-133.

Ferster D (1990b) X-and Y-mediated current sources in areas 17 and 18 of cat visual cortex. Vis Neurosci 4:135-145.

Ferster D, Jagadeesh B (1991) Nonlinearity of spatial summation in simple cells of areas 17 and 18 of cat visual cortex. J Neurophysiol 66:1667-1679.

Ferster D, Lindstrom S (1983) An intracellular analysis of geniculocortical connectivity in area 17 of the cat. J Physiol (Lond) 342:181215.

Foster KH, Gaska JP, Marcelja S, Pollen DA (1983) Phase relationships between adjacent simple cells in the feline visual cortex. J Physiol (Lond) 345:26P.

Fregnac Y, Imbert M (1978) Early development of visual cortical cells in normal and dark-reared kittens: relationship between orientation selectivity and ocular dominance. J Physiol (Lond) 278:27-44.

Fregnac Y, Imbert M (1984) Development of neuronal selectivity in the primary visual cortex of the cat. Physiol Rev 64:325-434.

Freund TF, Martin KAC, Somogyi P, Whitteridge D (1985) Innervation of cat visual areas 17 and 18 by physiologically identified $X$ and Y-type afferents. II. Identification of postsynaptic targets by GABA immunocytochemistry and Golgi impregnation. J Comp Neurol 242: 275-291.

Friauf E, Shatz CJ (1991) Changing patterns of synaptic input to subplate and cortical plate during development of visual cortex. $J$ Neurophysiol 66:2059-2071.

Friauf E, McConnell SK, Shaty CJ (1990) Functional synaptic circuits in the subplate during fetal and early postnatal development of cat visual cortex. J Neurosci 10:2601-2613

Gilbert CD (1977) Laminar differences in receptive field properties of cells in cat primary visual cortex. J Physiol (Lond) 268:391-421.

Gilbert CD, Hirsch JA, Wiesel TN (1990) Lateral interactions in visual cortex. Cold Spring Harbor Symp Quant Biol 55:663-677.

Guillery RW (1972) Binocular competition in the control of geniculate cell growth. J Comp Neurol 144:117-130.

Hahm JO, Langdon RB, Sur M (1991) Disruption of retinogeniculate afferent segregation by antagonists to NMDA receptors. Nature 351 $568-570$.

Hata Y. Tsumoto T, Sato H. Tamura H (1991) Horizontal interactions between visual cortical neurones studied by cross-correlation analysis in the cat. J Physiol (Lond) 441:593-614.

Hata Y, Tsumoto T, Sato H, Hagihara K, Tamura H (1993) Development of local horizontal interactions in cat visual cortex studied by cross-correlation analysis. J Neurophysiol 69:40-56.

Hebb DO (1949) The organization of behavior. New York: Wiley.

Heggelund P (1986) Quantitative studies of the discharge fields of single cells in cat striate cortex. J Physiol (Lond) 373:277-292.

Hess R. Negishi K. Creutzfeldt O (1975) The horizontal spread of intracortical inhibition in the visual cortex. Exp Brain Res 22:415419

Horton JC, Sherk H (1984) Receptive field properties in the cat's lateral geniculate nucleus in the absence of $\mathrm{ON}$-center retinal input. J Neurosci 4:374-380.

Hubcl DH (1982) Exploration of the primary visual cortex, 1955-78. Nature 299:515-524.

Hubel DH, Wiesel TN (1959) Receptive fields of single neurones in the cat's striate cortex. J Physiol (Lond) 148:574-591.

Hubel DH, Wiesel TN (1961) Integrative action in the cat's lateral geniculate body. J Physiol (Lond) 155:385-398.

Hubel DH, Wiesel TN (1962) Receptive fields, binocular interaction and functional architecture in the cat's visual cortex. J Physiol (Lond) $160: 106-154$

Hubel DH, Wiesel TN (1963) Receptive ficlds of cells in striate cortex of very young, visually inexperienccd kittens. J Neurophysiol 26:9941002

Hubel DH, Wiesel TN (1968) Receptive fields and functional architecture of monkey striate cortex. J Physiol (Lond) 195:215-243.

Hubcl DH, Wicscl TN (1974) Uniformity of monkey striate cortex: a parallel relationship between field size, scatter and magnification factor. J Comp Neurol 158:295-306.

Humphrey AL, Norton TT (1980) Topographic organization of the orientation column system in the striate cortex of the trec shrew (Tupaia glis). I. Microelectrode recording. J Comp Neurol 192:531547.

Humphrey AL, Skeen LC, Norton TT (1980) Topographic organization of the orientation column system in the striate cortex of the tree shrew (Tupaia glis). II. Deoxyglucose mapping. J Comp Neurol 192:549-566.

Humphrey AL, Sur M. Uhtrich DJ, Sherman SM (1985) Projection patterns of individual $X$ - and $Y$-cell axons from the lateral geniculate nucleus to cortical area 17 in the cat. J Comp Neurol 233:159-189.

Islcy MR, Rogers-Ramachandran DC, Shinkman PG (1990) Intcrocular torsional disparity and visual cortical development in the cat. J Neurophysiol 64:1352-1360.

Kaplan E, Marcus S, So YT (1979) Effects of dark adaptation on spatial and temporal properties of receptive fields in cat lateral geniculate nucleus. J Physiol (Lond) 294:561-579.

Kaplan E, Purpura K, Shapley RM (1987) Contrast affects the transmission of visual information through the mammalian lateral geniculate nucleus. J Physiol (Lond) 391:267-288.

LeVay S, McConnell SK (1982) ON and OFF layers in the lateral geniculate nucleus of the mink. Nature 300:350-351.

LeVay S, Nelson SB (1991) The columnar organization of visual cortex. In: Visual basis of visual function (Leventhal A, ed), pp 266315. Boca Raton, FL: CRC.

Leventhal $\Lambda G$, Schall JD (1983) Structural basis of orientation sensitivity of cat retinal ganglion cells. J Comp Neurol 220:465-475.

Levick WR. Thibos LN (1982) Analysis of orientation bias in cat retina. J Physiol (Lond) 329:243-261.

Lindstrom S, Wrobel A (1990) Frequency dependent corticofugal excitation of principal cells in the cat's dorsal lateral geniculate nucleus. Exp Brain Res 79:313-318.

Linsker R (1986a) From basic network principles to neural architecture: emergence of spatial-opponent cells. Proc Natl Acad Sci USA $83: 7508-7512$

Linsker R (1986b) From basic network principles to neural architecture: emergence of orientation-selective cells. Proc Natl Acad Sci UISA 83:8390-8394

Linsker R (1986c) From basic network principles to neural architecture: emergence of orientation columns. Proc Natl Acad Sci USA 83: $8779-8783$

Lund JS, Henry GH, MacQueen CL, Harvey AR (1979) Anatomical organization of the primary visual cortex (area 17) of the cat. A comparison with area 17 of the macaque monkey. J Comp Neurol $184: 599-618$ 
MacKay DJC, Miller KD (1990a) Analysis of I insker's applications of Hebbian rules to linear networks. Network 1:257-298.

MacKay DJC, Miller KD (1990b) Analysis of Linsker's simulations of Hebbian rules. Neural Comput 2:173-187.

Maffei L, Galli-Resta L (1990) Correlation in the discharges of neighboring rat retinal ganglion cells during prenatal life. Proc Natl Acad Sci USA 87:2861-2864.

Masland RH (1977) Maturation of function in the developing rabbit retina. J Comp Neurol 175:275-286.

Mastronarde DN (1983a) Correlated firing of cat retinal ganglion cells. I. Spontaneously active inputs to $X$ and $Y$ cells. J Neurophysiol 49 . 303-324

Mastronarde DN (1983b) Correlated firing of cat retinal ganglion cells. II. Responses of $\mathrm{X}$ - and $\mathrm{Y}$-cells to single quantal events. J Neurophysiol 49:325-349

Mastronarde DN (1987) Two classes of single-input X-cells in cat lateral geniculate nucleus. II. Retinal inputs and the generation of receptive field properties. J Neurophysiol 57:381-413.

McConnell SK, LeVay S (1984) Segregation of ON- and OFF-center afferents in mink visual cortex. Proc Natl Acad Sci USA 81:15901593.

Meister M, Wong ROL, Baylor DA, Shatz CJ (1991) Synchronous bursts of action-potentials in ganglion cells of the developing mammalian retina. Science 252:939-943.

Miller KD (1989a) Correlation-based mechanisms in visual cortex: theoretical and experimental studies. PhD thesis, Stanford University Medical School.

Miller KD (1989b) Orientation-selective cells can emerge from a Hebbian mechanism through interactions between ON- and OFF-center inputs. Soc Neurosci Abstr 15:794.

Miller KD (1990a) Correlation-based models of neural development. In: Neuroscience and connectionist theory (Gluck MA, Rumelhart DE, eds), pp 267-353. Hillsdale, NJ: Erlbaum.

Miller KD (1990b) Cortical organization of orientation selectivity emerges from interactions between $\mathrm{ON}$ - and OFF-center inputs. Soc Neurosci Abstr 16:798.

Miller KD (1990c) Derivation of linear Hebbian equations from a nonlinear Hebbian model of synaptic plasticity. Neural Comput 2:321333.

Miller KD (1992a) Development of orientation columns via competition between ON- and OFF-center inputs. Neuroreport 3:73-76.

Miller KD (1992b) Developmental ON/OFF competition can quantitatively account for preferred spatial frequencies of cortical simple cells. Soc Neurosci Abstr 18:1306.

Miller KD (1992c) Models of activity-dependent neural development. Semin Neurosci 4:61-73.

Miller KD, MacKay DJC (1994) The role of constraints in Hebbian learning. Neural Comput 6:98-124.

Miller KD, Stryker MP (1990) The development of ocular dominance columns: mechanisms and models. In: Connectionist modeling and brain function: the developing interface (Hanson SJ, Olson CR, eds), pp 255-350. Cambridge, MA: MIT Press/Bradford.

Miller KD, Keller JB, Stryker MP (1989) Ocular dominance column development: analysis and simulation. Science 245:605-615.

Movshon JA, Van Sluyters RC (1981) Visual neural development. Annu Rev Psychol 32:477-522.

Movshon IA. Thompson ID. Tolhurst DJ (1978a) Spatial summation in the receptive fields of simple cells in the cat's striate cortex. J Physiol (Lond) 283:53-77.

Movshon JA, Thompson ID, Tolhurst DJ (1978b) Spatial and temporal contrast sensitivity of neurones in areas 17 and 18 of the cat visual cortex. J Physiol (Lond) 283:101-120.

Mullikan WH, Jones JP, Palmer LA (1984a) Receptive-field properties and laminar distribution of $\mathrm{X}$-like and $\mathrm{Y}$-like simple cells in cat area 17. J Neurophysiol 52:351-371.

Mullikan WH, Jones JP, Palmer LA (1984b) Periodic simple cells in cat area 17. J Neurophysiol 52:372-387.

Murray JD (1989) Mathematical biology. New York: Springer

Nelson SB (1991) Temporal interactions in the cat visual cortex. II. Suppressive and facilitatory effects in the lateral geniculate nucleus. J Neurosci 11:357-368.

Niebur E, Wörgötter F (1993) Orientation column structures from first principles. In: Computation and neural systems 92, Chap 62. Norwell, MA: Kluwer.

Obermayer K, Ritter H, Schulten K (1990) A principle for the for- mation of the spatial structure of cortical feature maps. Proc Natl Acad Sci USA 87:8345-8349.

Obermayer K, Blasdel GG, Schulten K (1992) A statistical mechanical analysis of self-organization and pattern formation during the development of visual maps. Phys Rev [A] 45:7568-7589.

Palmer LA, Davis TL (1981) Receptive-field structure in cat striate cortex. J Neurophysiul 46:260-276.

Peinado A, Yuste R, Katz LC (1993) Extensive dye coupling between rat neocortical neurons during the period of circuit formation. Neuron 10:103-114.

Penrose R (1979) The topology of ridge systems. Ann Hum Genet 42: 435-444.

Perez R, Glass L, Shlaer R (1975) Development of specificity in the cat visual cortex. J Math Biol 1:275-288.

Pollen DA, Ronner SF (1981) Phase relationships between adjacent simple cells in the visual cortex. Science 212:1409-1411.

Press WH, Flannery BP, Teukolsky SA, Vetterling WT (1988) Numerical recipes in $C$. Cambridge: Cambridge UP.

Raczkowski D, Fitzpatrick D (1990) Terminal arbors of individual, physiologically identified geniculocortical axons in the tree shrew's striate cortex. J Comp Neurol 302:500-514.

Ramachandran VS, Clarke PGH, Whitteridge D (1977) Cells selective to binocular disparity in the cortex of newborn lambs. Nature 268 : 333-335.

Rojer AS, Schwartz EL (1990) Cat and monkey cortical columnar patterns modeled by bandpass-filtered 2D white noise. Biol Cybern 62:381-391.

Schall JD, Vitek DJ, Leventhal AG (1986) Retinal constraints on orientation specificity in cat visual cortex. J Neurosci 6:823-836

Schiller PH (1982) Central connections of the retinal ON and OFF pathways. Nature 297:580-583.

Schiller PH (1984) The connections of the retinal ON and OFF pathways to the lateral geniculate nucleus in the monkey. Vision Res 24: 923-932.

Schiller PH, Malpeli JG (1978) Functional specificity of lateral geniculate nucleus laminae of the rhesus monkey. J Neurophysiol 41:788797.

Schiller PH, Finlay BL, Volman SF (1976) Quantitative studies of single-cell properties in monkey striate cortex. I. Spatiotemporal organization of receptive fields. J Neurophysiol 39:1288-1319.

Sejnowski TJ (1977) Storing covariance with nonlinearly interacting neurons. J Math Biol 4:303-321.

Shatz. CJ (1990) Impulse activity and the patterning of connections during CNS development. Neuron 5:745-756.

Shatz CJ, Luskin MB (1986) The relationship between the geniculocortical afferents and their cortical target cells during development of the cat's primary visual cortex. J Neurosci 6:3655-3668.

Sherk H, Horton JC (1984) Receptive field properties in the cat's area 17 in the absence of ON-center geniculate input. J Neurosci 4:381393

Shoham D, Ullman S, Grinvald A (1991) Charactcrization of dynamic patterns of cortical activity by a small number of principal components. Soc Neurosci Abstr 17:1089.

Sillito AM, Kemp IA (1983) The influence of GABAergic inhibitory processes on the receptive field structure of $X$ and $Y$ cells in cat dorsal lateral geniculate nucleus (dLGN). Brain Res 277:63-77.

Silvcrman MS, Grosof DH, DeValois RL, Llfar SD (1989) Spatial frequency organization in primate striate cortex. Proc Natl Acad Sci USA 86:711-715.

Soodak RE (1987) The retinal ganglion cell mosaic defines orientation columns in striate cortex. Proc Natl Acad Sci USA 84:3936-3940.

Soodak RE (1991) Reverse-Hebb plasticity leads to optimization and association in a simulated visual cortex. Vis Neurosci 6:507-518.

Soodak RE, Shapley RM, Kaplan E (1987) Linear mechanism of orientation tuning in the retina and lateral geniculate nucleus of the cat. J Neurophysiol 58:267-275.

Sretavan DW, Shatz CJ (1987) Axon trajectories and pattern of terminal arborization during the prenatal development of the cat's retinogeniculate pathway. J Comp Neurol 255:386-400.

Stevens JK, Gerstein GL (1976) Interactions between cat lateral geniculate neurons. J Neurophysiol 39:239-256.

Stryker MP, Strickland SL (1984) Physiological segregation of ocular dominance columns depends on the pattern of afferent electrical activity. Invest Ophthalmol Vis Sci [Suppl] 25:278. 
Stryker MP, 7ahs KR (1983) ON and OFF sublaminae in the lateral geniculate nucleus of the ferret. J Neurosci 3:1943-1951.

Stryker MP, Chapman B, Miller KD, Zahs KR (1990) Experimental and theoretical studies of the organization of afferents to single orientation columns in visual cortex. Cold Spring Harbor Symp Quant Biol 55:515-527.

Swindale NV (1982) A model for the formation of orientation columns. Proc R Soc Lond [Biol] 215:211-230.

Swindale NV, Matsubara JA, Cynader MS (1987) Surface organization of orientation and direction selectivity in cat area 18 . J Neurosci 7:1414-1427.

Tanaka K (1983) Cross-correlation analysis of geniculostriate neuronal relationships in cats. J Neurophysiol 49:1303-1318.

Tanaka S (1990) Theory of self-organization of cortical maps: mathematical framework. Neural Networks 3:625-640.

Tanaka S (1992) A mathematical model for the self-organization of orientation columns in visual cortex. Neuroreport 3:69-72.

Tootell RBH, Silverman MS, Hamilton SL, Switkes E, DeValois RL (1988) Functional anatomy of macaque striate cortex. V. Spatial frequency. J Neurosci 8:1610-1624.

Toyama K, Kimura M, Tanaka K (1981) Cross-correlation analysis of interneuronal connectivity in cat visual cortex. J Neurophysiol 46: 191-201.

Ts'o DY, Frostig RD, Lieke E, Grinvald A (1990) Functional organization of primate visual cortex revealed by high resolution optical imaging of intrinsic signals. Science 249:417-423.

Tsumoto T, Suda K (1982) Laminar differences in development of afferent innervation to striate cortex neurones in kittens. Exp Brain Res 45:433-446.

Turing AM (1952) The chemical basis of morphogenesis. Philos Trans R Soc Lond [Biol] 237:37-72.

Tusa RJ, Palmer LA, Rosenquist AC (1978) The retinotopic organization of area 17 (striate cortex) in the cat. J Comp Neurol 177: 213-235.
Virsu V, Lee BB, Creutzfeldt OD (1977) Dark adaptation and receptive field organisation of cells in the cat lateral geniculate nucleus. Exp Brain Res 27:35-50.

von der Malsburg C (1973) Self-organization of orientation selective cells in the striate cortex. Kybernetik 14:85-100.

von der Malsburg C, Willshaw DJ (1976) A mechanism for producing continuous neural mappings: ocularity dominance stripes and ordered retino-tectal projections. Exp Brain Res [Suppl] 1:463-469

Wiesel TN, Hubel DH (1965) Comparison of the effects of unilateral and bilateral eye closure on cortical unit responses in kittens. J Neurophysiol 28:1029-1040.

Wiesel TN, Hubel DH (1974) Ordered arrangement of orientation columns in monkeys lacking visual experience. J Comp Neurol 158: 307-318.

Wong ROL, Meister M, Shatz.CJ (1991) Spontaneous correlated hursiing activity of retinal ganglion cells is unique to the period of axonal segregation. Soc Neurosci Abstr 17:186.

Wörgötter F, Eysel UT (1991a) Axial responses in visual cortical cells: spatio-temporal mechanisms quantified by Fourier components of cortical tuning curves. Exp Brain Res 83:656-664.

Wörgötter F, Eysel UT (1991b) Topographical aspects of intracortical excitation and inhibition contributing to orientation specificity in area 17 of the cat visual cortex. Eur J Neurosci 3:1232-1244.

Wörgötter F, Koch C (1991) A detailed model of the primary visual pathway in the cat: comparison of afferent excitatory and intracortical inhibitory connection schemes for orientation selectivity. J Neurosci 11:1959-1979.

Wörgötter F, Gründel O, Eysel UT (1990) Quantification and comparison of cell properties in cat's striate cortex determined by different types of stimuli. Eur J Neurosci 2:928-941.

Yuste R, Peinado A, Katz LC (1992) Neuronal domains in developing neocortex. Science 257:666-669.

Zahs KR, Stryker MP (1988) Segregation of ON and OFF afferents to ferret visual cortex. J Neurophysiol 59:1410-1429. 